\title{
Electrochemically enabled Chan-Lam couplings of aryl boronic acids and anilines
}

Ryan P. Wexler, Philippe Nuhant, Timothy J. Senter, Zachary J. Gale-Day*

Vertex Pharmaceuticals, Inc., 50 Northern Avenue, Boston, MA 02210

\section{TABLE OF CONTENTS}

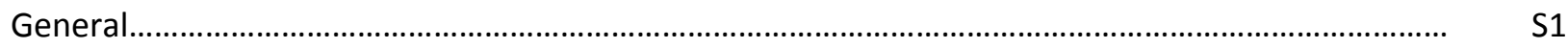

Expanded optimization table............................................................................................. S2

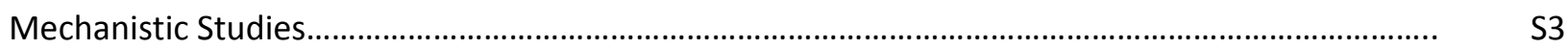

General Procedure for the preparation of bis-aryl anilines............................................................. S11

Synthesis and characterization data of products............................................................................ S11

Procedure for the photochemical Chan-Lam of 3m........................................................................ S20

Procedure for the synthesis of $\mathbf{3 a}$ on $\mathrm{mmol}$ scale ..................................................................... S20

Copies of NMR spectra............................................................................................................... S21

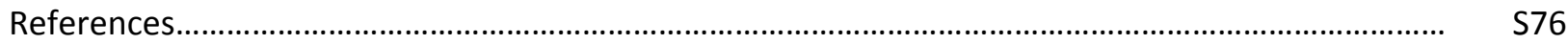

General information: ${ }^{1 \mathrm{H}}$ and ${ }^{13} \mathrm{C}$ NMR spectra were recorded on a Brunker $300 \mathrm{Mhz}, 76 \mathrm{Mhz}$ spectrometer in $\mathrm{CDCl}_{3}$. Chemical shifts were reported in parts per million (ppm) from $\mathrm{Me}_{4} \mathrm{Si}$ using the solvent resonance as the internal standard $\left(\mathrm{CDCl}_{3} \delta=7.26 \mathrm{ppm}\right)$ for ${ }^{1} \mathrm{H} \mathrm{NMR}$ and $\left(\mathrm{CDCl}_{3} \delta=77.16 \mathrm{ppm}\right)$ for ${ }^{13} \mathrm{C}$ NMR. Flash Chromotography was performed using CombiFlash Rf+ Lumen Automated Flash Chromatography System with $200 \mathrm{~mL} / \mathrm{min}$ max flow, 200 psi, with integrated ELSD and 200-400 nm UV variable wavelength detector and a $12 \mathrm{~g}$ HP Silica RediSep Rf gold column. Solvents were evaporated by either Biotage V10 touch or rotary evaporator. Low-Resolution Mass Spectrometry analyses were conducted on Waters Acquity UPLC (Acquity Binary Solvent Manager, 2777C-Autosampler, Acquity PDA, Acquity ELS and Acquity Column Manager) and Waters Acquity SQ systems from Waters Corporation, Milford, MA. Signal acquisition conditions included: Waters Acquity HSS T3, 2.1mmx50mm, C18, 1.7 $\mu \mathrm{m}$; Column Temperature $60{ }^{\circ} \mathrm{C}$ as the column; $0.1 \%$ formic acid in water $(\mathrm{v} / \mathrm{v})$ as the mobile phase $\mathrm{A} ; 0.1 \%$ formic acid in acetonitrile (v/v) as the mobile phase $\mathrm{B} ; 1.25 \mathrm{~mL} / \mathrm{min}$ as the flow and $\mathrm{ESCl}(\mathrm{ESI}+/-, \mathrm{APCl}+/-)$, $100-2000 \mathrm{~m} / \mathrm{z}$ scan, $0.4 \mathrm{sec}$ scan time, Centroid as the MS method. All electrolysis and cyclic volttamtetric experiments were carried out in IKA ElectraSyn 2.0 machines utilizing IKA copper electrodes (99.9\%) as both the anode and the cathode.

Reagents: Commercial reagents and solvents were used without further purification. 
Table 1. Expanded optimization table ${ }^{a}$

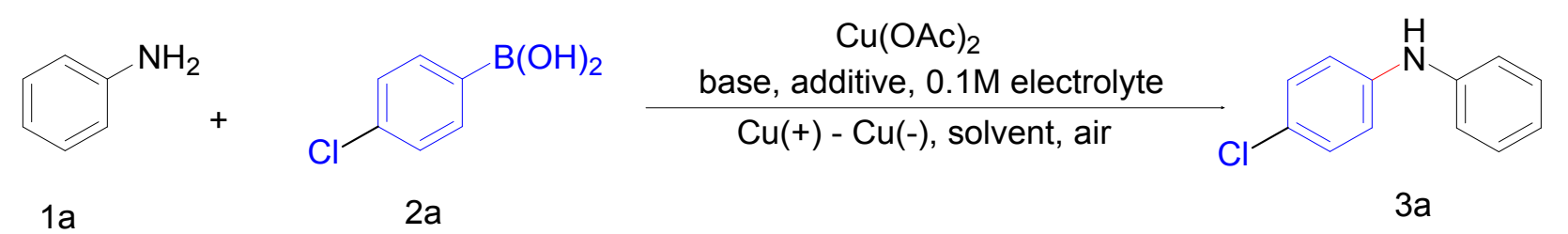

\begin{tabular}{|c|c|c|c|c|c|}
\hline Entry & Solvent $(4 \mathrm{~mL})$ & Electrolyte $(0.1 \mathrm{M})$ & Base (1.2 eq) & Additive (20 mol\%) & Yield $^{b}$ \\
\hline 1 & $\mathrm{ACN}$ & $\mathrm{NBu}_{4} \mathrm{ClO}_{4}$ & --- & --- & $48 \%$ \\
\hline 2 & $\mathrm{ACN}$ & $\mathrm{NBu}_{4} \mathrm{BF}_{4}$ & --- & --- & $35 \%$ \\
\hline 3 & $\mathrm{ACN}$ & $\mathrm{LiClO}_{4}$ & --- & --- & $7 \%$ \\
\hline 4 & $\mathrm{ACN}$ & $\mathrm{LiCl}$ & --- & --- & $9 \%$ \\
\hline 5 & ACN:Toluene (1:1) & $\mathrm{NBu}_{4} \mathrm{ClO}_{4}$ & --- & --- & $35 \%$ \\
\hline 6 & $\mathrm{ACN}$ & $\mathrm{NBu}_{4} \mathrm{ClO}_{4}$ & 2,6-Lutidine & --- & $68 \%$ \\
\hline 7 & $\mathrm{ACN}$ & $\mathrm{NBu}_{4} \mathrm{ClO}_{4}$ & Potassium Carbonate & --- & $28 \%$ \\
\hline 8 & $\mathrm{ACN}$ & $\mathrm{NBu}_{4} \mathrm{ClO}_{4}$ & Cesium Carbonate & --- & $18 \%$ \\
\hline 9 & $\mathrm{ACN}$ & $\mathrm{NBu}_{4} \mathrm{ClO}_{4}$ & 2,6-Lutidine & Myristic Acid & $56 \%$ \\
\hline 10 & $\mathrm{ACN}$ & $\mathrm{NBu}_{4} \mathrm{ClO}_{4}$ & 2,6-Lutidine & Tris(4-Bromophenyl)amine & $72 \%$ \\
\hline 11 & ACN & $\mathrm{NBu}_{4} \mathrm{ClO}_{4}$ & 2,6-Lutidine & Triphenylamine & $62 \%$ \\
\hline 13 & $\mathrm{ACN}$ & $\mathrm{NBu}_{4} \mathrm{ClO}_{4}$ & 2,6-Lutidine & 1,1'-Dibromoferrocene & $64 \%$ \\
\hline 14 & $\mathrm{ACN}$ & $\mathrm{NBu}_{4} \mathrm{ClO}_{4}$ & 2,6-Lutidine & TEA & $98 \%$ \\
\hline 15 & $\mathrm{ACN}$ & $\mathrm{NBu}_{4} \mathrm{ClO}_{4}$ & TEA ( 2 equiv.) & --- & $69 \%$ \\
\hline 15 & $\mathrm{ACN}$ & $\mathrm{NBu}_{4} \mathrm{ClO}_{4}$ & 2,6-Lutidine & Pyridine & $80 \%$ \\
\hline 16 & $\mathrm{ACN}$ & $\mathrm{NBu}_{4} \mathrm{ClO}_{4}$ & 2,6-Lutidine & Boric Acid & $30 \%$ \\
\hline
\end{tabular}

${ }^{a}$ Reaction conditions: aniline $1 \mathrm{a}(0.55 \mathrm{mmol}), 2 \mathrm{a}(0.71 \mathrm{mmol}), 0.1 \mathrm{M} \mathrm{NBu}_{4} \mathrm{ClO}_{4} / \mathrm{ACN}(4 \mathrm{~mL})$, additive $(0.11$ mmol, 0.2 equiv.), base ( $0.66 \mathrm{mmol}, 1.2$ equiv), $\mathrm{Cu}(\mathrm{OAc})_{2}(0.11 \mathrm{mmol}, 20 \mathrm{~mol} \%), \mathrm{RT}$, undivided cell, air, 20h. ${ }^{b}$ Yields determined by NMR integration using 1,4-dinitrobenzene as an internal standard. 


\section{Mechanistic studies:}

All cyclic voltammograms were recorded using the IKA ElectroSyn 2.0 and included CV Kit. The working electrode was glassy carbon with $d=3.0 \mathrm{~mm}$. A platinum plates counter was used in all cases and referenced against the $\mathrm{Ag} / \mathrm{AgCl}$ electrode. The working electrode was polished using an alumina suspension in water (1.0 micron particle size) and cleaned thoroughly with both water and acetone before use. Tetrabutylammonium perchlorate $(0.1 \mathrm{M})$ was used was used as the supporting electrolyte in all cases. All samples were stirred under oxygen for 20 minutes before sparging with nitrogen for 5 minutes preceding spectra recorded. All spectra were recorded with a $50 \mathrm{mV} \mathrm{s}^{-1}$ scan rate with a forward sweep. All analysis and graphing of CV data was done using Python 2.7 and matplotlib. All CV studies were done using aniline 1a and boronic acid 2a unless otherwise noted and setup as indicated in the general procedure with the proper equivalencies excluding the relevant copper salt which was kept to 5 $\mathrm{mM}$ in all cases.

Discusion of mechanistic studies: In order to inverstigate the mechanism of our electrochemically mediate Chan-Lam reaction a series of CV studies were conducted and shown below. Initially to our surprise, there was no evidence of any oxidative behavior on the time-scale of the CV studies of the reaction mixture, but there was a strong quasi-reversible reduction observed, $E_{1 / 2}{ }^{\text {red }}=-0.35 \mathrm{~V}$ ( $\mathrm{vs}$ $\mathrm{Ag} / \mathrm{AgCl}$ ) (SI Figure 1). We hypothesized that this reduction peak could correspond to one of two processes, either the $\mathrm{Cu}^{11 / 1}$ redox couple of the active catalyst or the $\mathrm{Cu}^{1 / 0}$ redox couple of the $\mathrm{Cu}^{1}$ produced through the catalytic cycle. To rule out the direct reduction of Cull CV was conducted on a copper (II) acetate solution with triethylamine and 2,6-lutidine added to most closely mimic reaction conditions, without the possibility of catalytic turnover to $\mathrm{Cu}^{\prime}$. No redox behavior was observed (SI Figure 1), making the direct reduction of $\mathrm{Cu}^{\prime \prime}$ unlikely to be the redox active species. To test whether it was the $\mathrm{Cu}^{1 / 0}$ redox couple being observed the $\mathrm{Cu}^{\prime \prime}$ acetate was replaced with $\mathrm{Cu}^{\prime}$ acetate keeping the other conditions constant. A distinct reduction peak $E_{1 / 2}{ }^{\text {red }}=-0.53 \mathrm{~V}$ ( $\mathrm{vs} \mathrm{Ag} / \mathrm{AgCl}$ ). This behavior was extremely similar to the, observed after aging the reaction mixure and presumably completing a catalytic turnover resulting in the formation of $\mathrm{Cu}^{\prime}$. The results of this experiment confirmed that the reduction was most likely the $\mathrm{Cu}^{1 / 0}$ redox couple. Interestingly the reverse sweep showed a strong quasireversible oxidation peak, consistent with the stripping of the $\mathrm{Cu}^{0}$ thin film from the electrode surface. With this initial information in hand the effect of the additives on the redox behavior of the system was investigated. The removal of either TEA (SI Figure 2) or 2,6-lutidine (SI Figure 3) increases the reduction potential to $E_{1 / 2}{ }^{\text {red }}=-0.55 \mathrm{~V}(\mathrm{vs} \mathrm{Ag} / \mathrm{AgCl})$ and $\mathrm{E}_{1 / 2}{ }^{\text {red }}=-0.46 \mathrm{~V}(\mathrm{vs} \mathrm{Ag} / \mathrm{AgCl}$ ) respectively, while the complete removal of all additives (SI Figure 4) results in an $E_{1 / 2}{ }^{\text {red }}=-0.53 \mathrm{~V}(\mathrm{vs} \mathrm{Ag} / \mathrm{AgCl}$ ). It seems that TEA plays a minor role in reducing the reduction potential of the $\mathrm{Cu}^{\prime}$ species, while using 2,6-lutidine alone has no effect. However, when combined, there appears to be a synergistic effect in reducing the reduction potential of the $\mathrm{Cu}^{\prime}$ species. The AUC of the oxidation peak intitially seemed to indicate a possible two-electron oxidation regenerating the catalytically active $\mathrm{Cu}^{\prime \prime}$ species. To determine whether this was the case a set of four control experiments was run (SI Scheme 1). As discussed in the main body of the paper the reaction with no current applied results in a reduced yield of $\mathbf{5 4 \%}$ (SI Scheme $\mathbf{1} \mathbf{b}$ ). Next we ran the electrochemical conditions under nitrogen (SI Scheme $\mathbf{1} \mathbf{c}$ ) and observed a yield consistent with the reaction with no current and under nitrogen(SI Scheme d) $\mathbf{1 5 \%}$ consistent with one catalytic cycle. This indicated the the oxidation peak corrisponds to the $\mathrm{Cu}^{\circ} / \mathrm{Cu}^{\prime}$ redox couple. This implies that the yield improvement observed is a result of the selective removal of $\mathrm{Cu}^{\prime}$ from the reaction mixture, limiting the side-product formation, perserving the boronic acid for reactivity and allowing the reaction solution to saturate with oxygen. To the best of our knowledge, this effect is unreported in the literature and was beyond the scope of this work to investigate. Overall, this evidence may support the hypothesized mechanism reported in this publication. 
Figure 1. Cyclic voltamogram of copper (II) acetate background with 2-6-lutidine and triethylamine, normal reaction conditions and normal reaction conditions with copper (I) acetate substituted for copper (II) acetate.

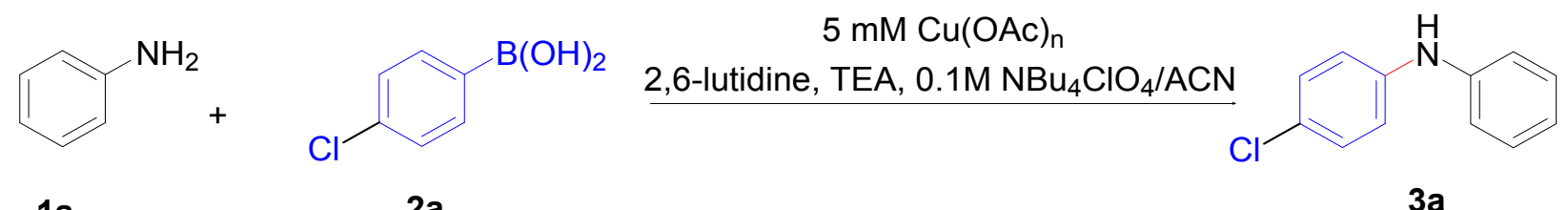

$1 a$

2a

$3 a$

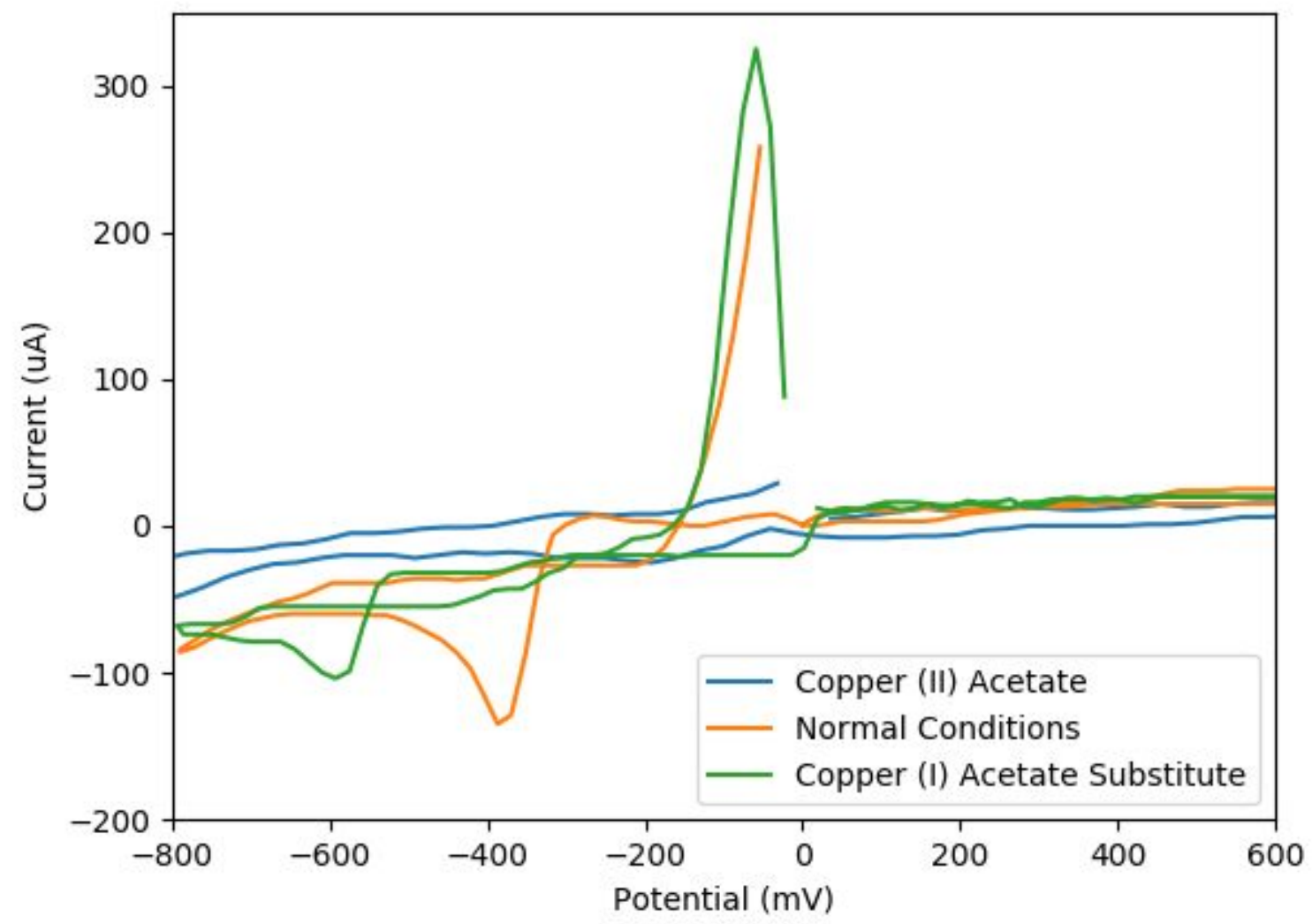


Figure 2. Cyclic voltamogram excluding TEA from the reaction mixture.

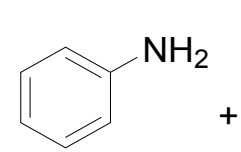

$1 a$

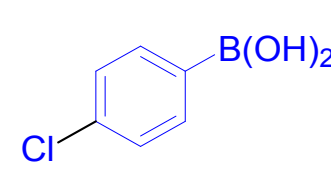

$2 a$

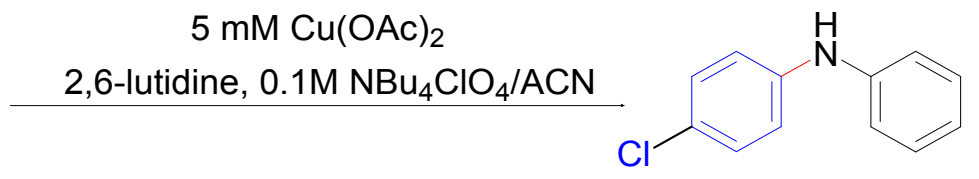

3a

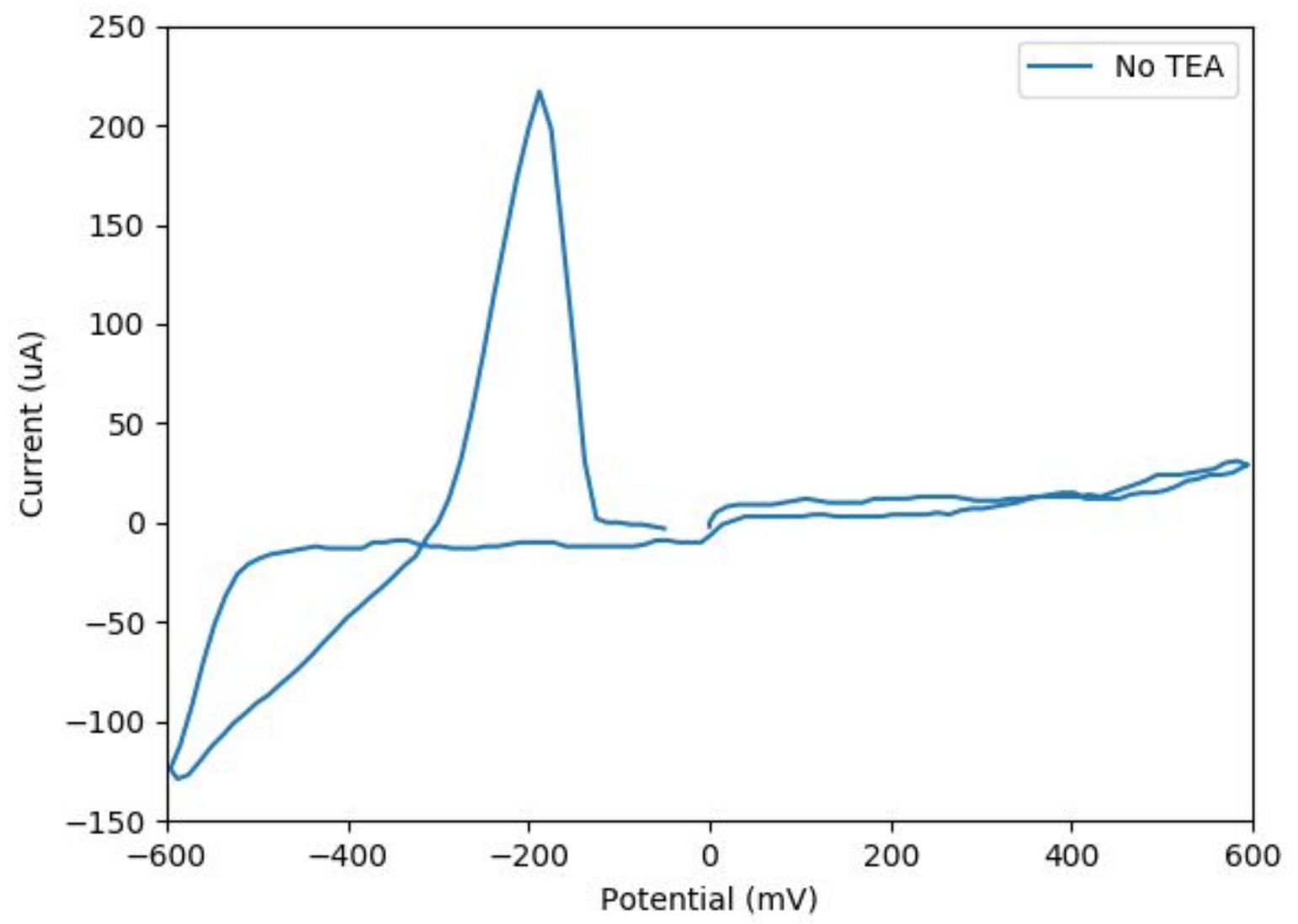


Figure 3. Cyclic voltamogram excluding 2,6-Lutidine from the reaction mixture

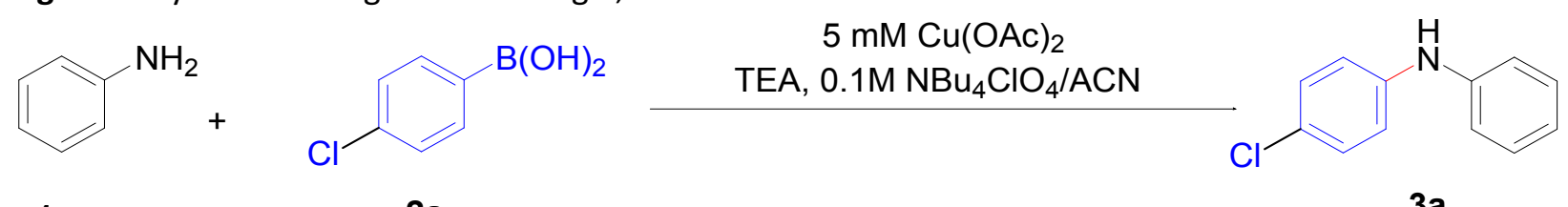

$1 a$

$2 a$

$3 a$

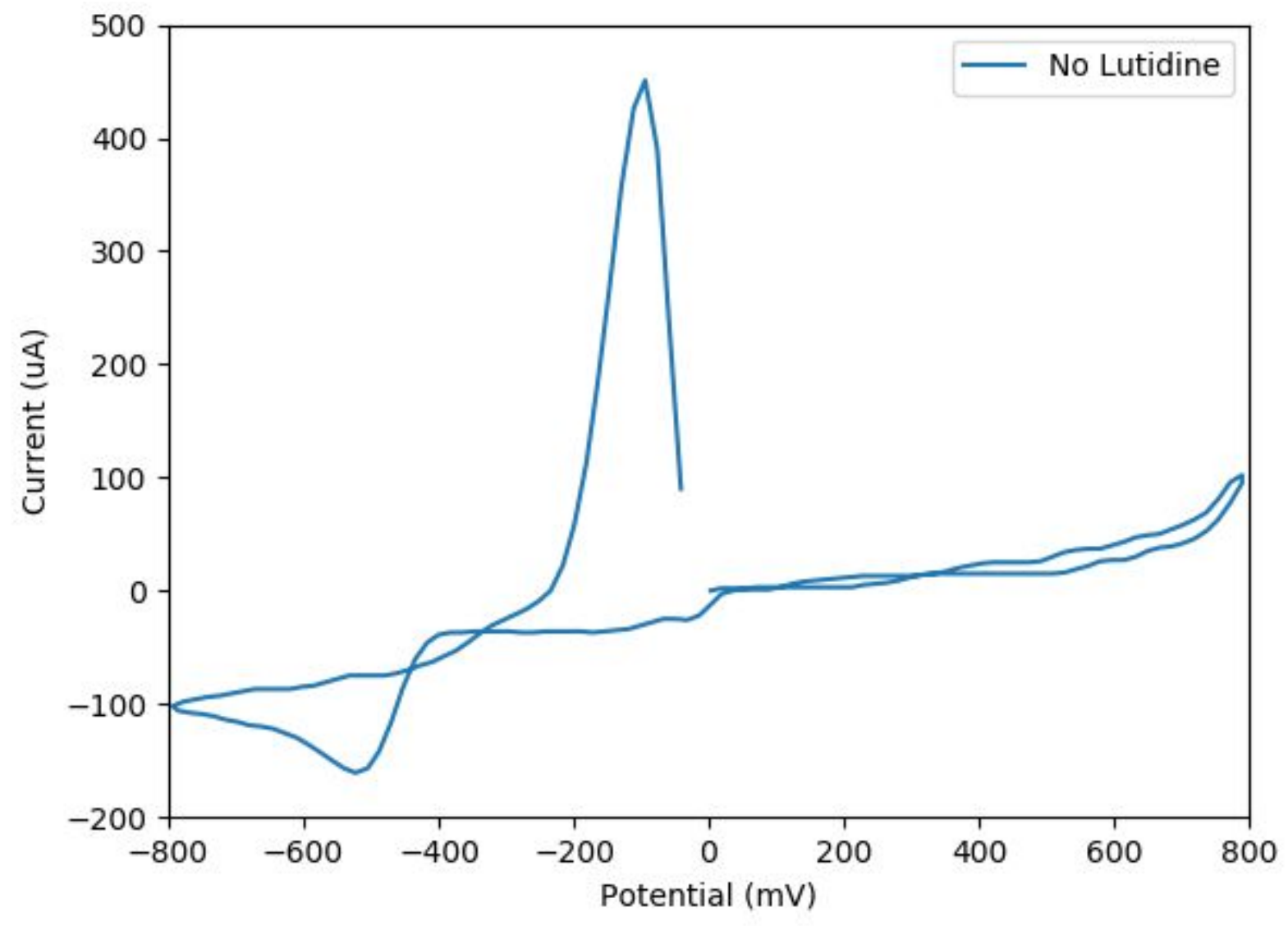


Figure 4. Cyclic voltamogram excluding both 2,6-Lutidine and TEA from the reaction mixture.

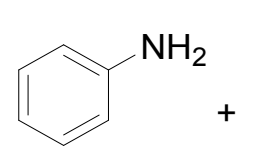

1a

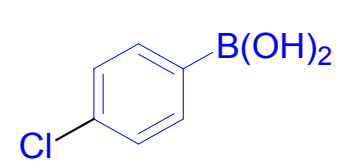

$2 a$

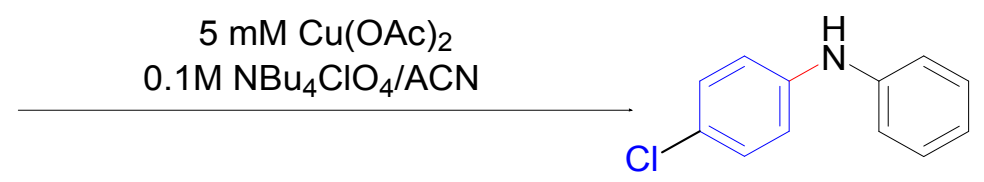

$3 a$

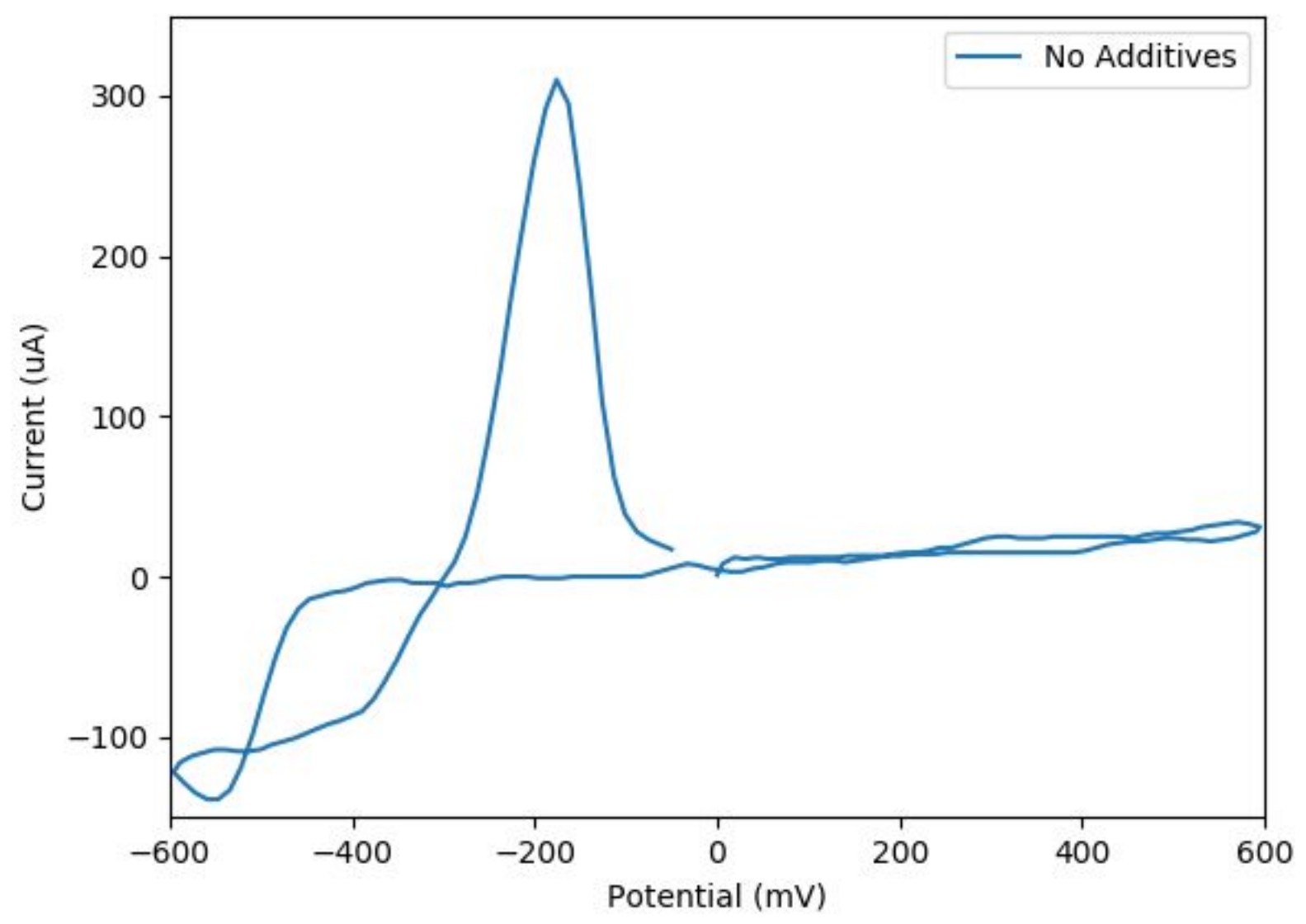


Discussion of side product profile: When control reactions were run with identical conditions without applying current, different side product were observed. Highlighted in figure $\mathbf{5}$ is an example where increased amount of para-chlorophenol is formed, supporting the mechanistic hypothesis.

Figure 5. Comparison of side product profile of electrochemical vs. control reaction
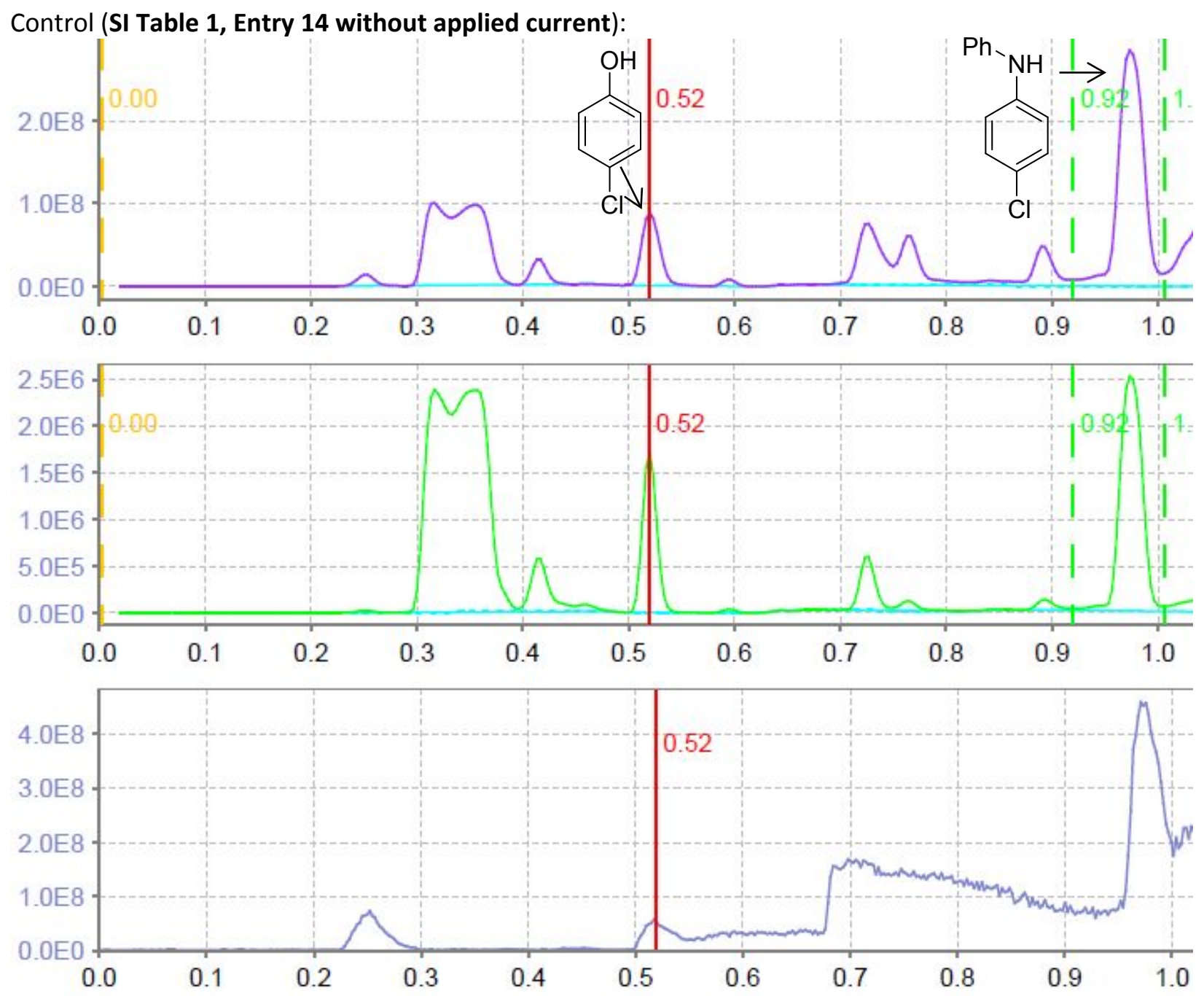

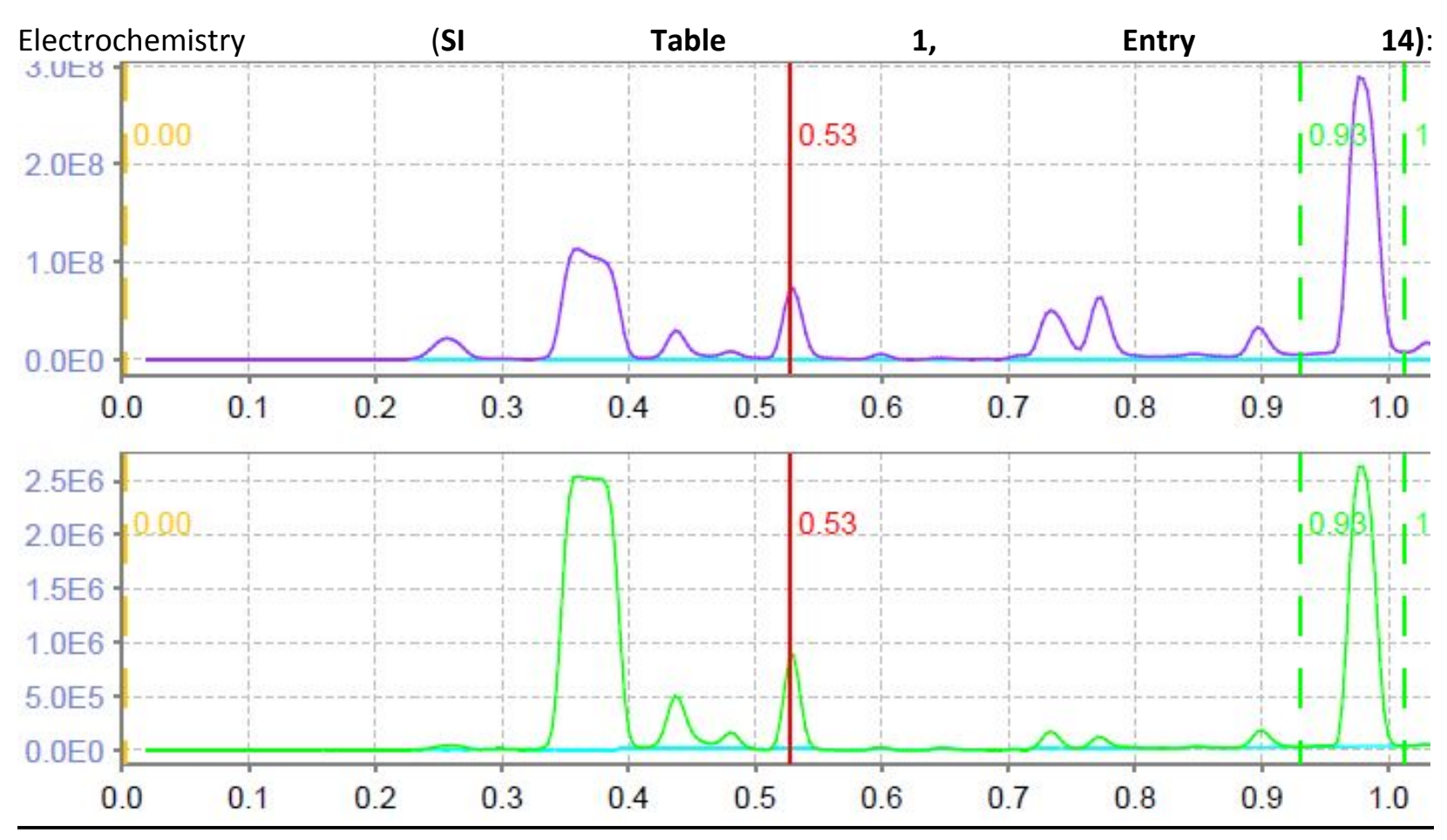


\section{Scheme 1. Control Experiments ${ }^{\mathrm{a}}$.}

\section{Normal Conditions:}

a) Normal Conditions:
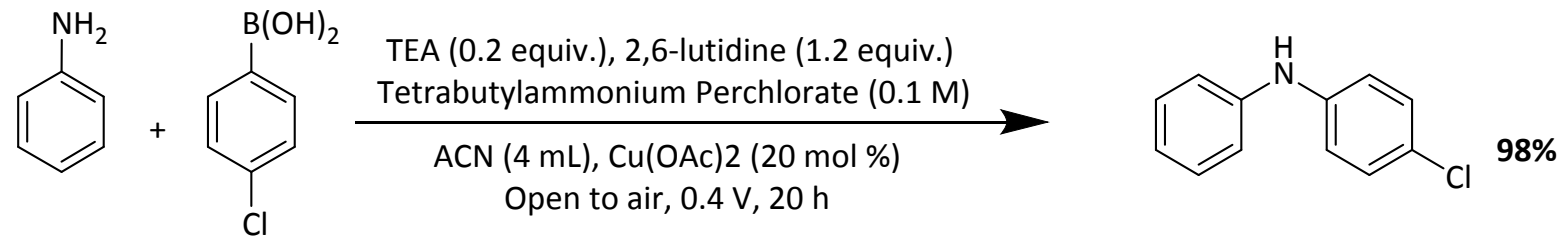

$1 a$

$2 a$

$3 a$

b) No current applied:<smiles>Nc1ccccc1</smiles>

$1 a$<smiles>Oc1ccc(Br)cc1</smiles>

$2 a$
TEA (0.2 equiv.), 2,6-lutidine (1.2 equiv.)

Tetrabutylammonium Perchlorate (0.1 M)

ACN (4 mL), Cu(OAc)2 (20 mol \%)

Open to air, $20 \mathrm{~h}$<smiles>O=S(=O)(O)c1ccc(Nc2ccccc2)cc1</smiles>

3a

c) Normal Conditions under nitrogen:

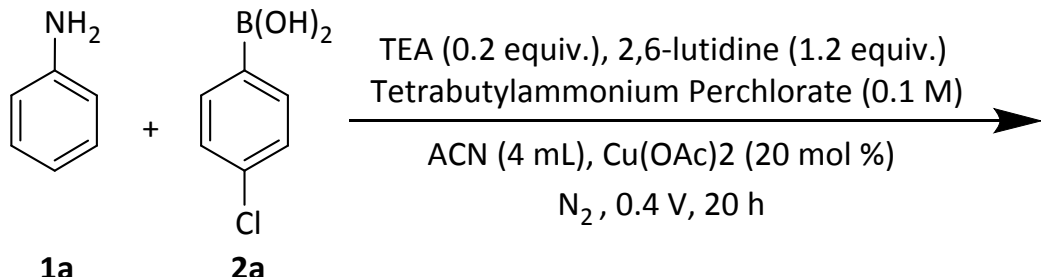<smiles>Clc1ccc(Nc2ccccc2)cc1</smiles>

$15 \%$

1a

$2 a$ 3a

d) No current applied under nitrogen:

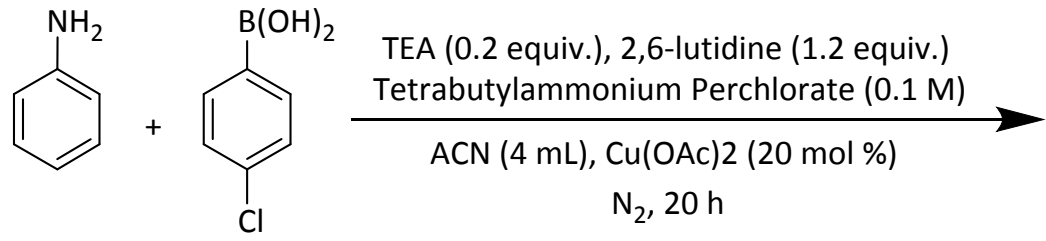

$1 a$

$2 a$<smiles>O=S(=O)([Sb])c1ccc(Nc2ccccc2)cc1</smiles>

$3 a$

aYields determined by NMR integration using 1,4-dinitrobenzene as an internal standard. 


\section{General Procedure for the preparation of bis-aryl anilines}

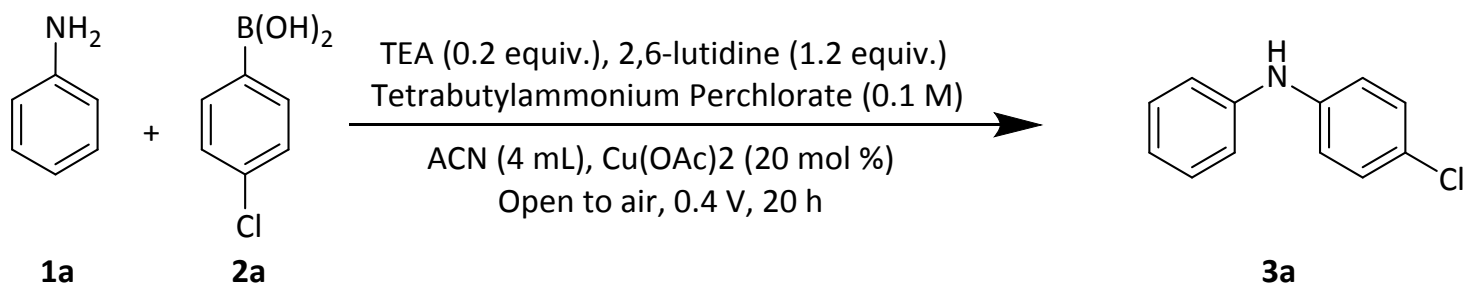

In a $10 \mathrm{~mL}$ IKA-Works ElectraSyn vial was added $\mathrm{Cu}(\mathrm{OAc})_{2}(20 \mathrm{mg}, 0.11 \mathrm{mmol}, 20 \mathrm{~mol} \%$ ), tetrabutylammonium perchlorate $(137 \mathrm{mg}, 0.40 \mathrm{mmol}, 1 \mathrm{M})$, triethylamine $(15 \mu \mathrm{L}, 0.11 \mathrm{mmol}, 0.2$ equiv.), 2,6-lutidine (76 $\mu \mathrm{L}, 0.66 \mathrm{mmol}), \mathrm{ACN}(4 \mathrm{~mL})$, arylboronic acid $2 \mathrm{a}(112 \mathrm{mg}, 0.71 \mathrm{mmol})$, and aniline derivative 1a $(50 \mu \mathrm{L}, 0.55 \mathrm{mmol})$. The vial was left open to atmosphere and was stirred for 20 hours under a potential of $0.40 \mathrm{~V}$. The crude reaction mixture was then diluted with ethyl acetate and passed through a $7 \mathrm{~g}$ silica plug to remove copper. Next, the reaction mixture was blown down, taken up in minimal DCM, and purified by flash chromatography on a $12 \mathrm{~g}$ silica column (0-40\% EtOAc/Heptane). After removal of solvent, $N, N$-diarylamine $3 a(101 \mathrm{mg}, 0.50 \mathrm{mmol}, \mathbf{9 0 \%})$ eluted as a yellow oil.

The control reaction was run following an identical reaction procedure except that electrolysis was not applied. For the workup, the crude reaction mixture was also diluted with ethyl acetate and passed through a $7 \mathrm{~g}$ silica gel plug to remove copper. However, after removal of solvent, $50 \mathrm{mg}$ of the NMR standard 1,4-dinitrobenzene were taken up in excess $\mathrm{CDCl}_{3}$ along with the reaction mixture. NMR analysis using the internal standard then afforded the yield.

\section{Synthesis and characterization data of products}

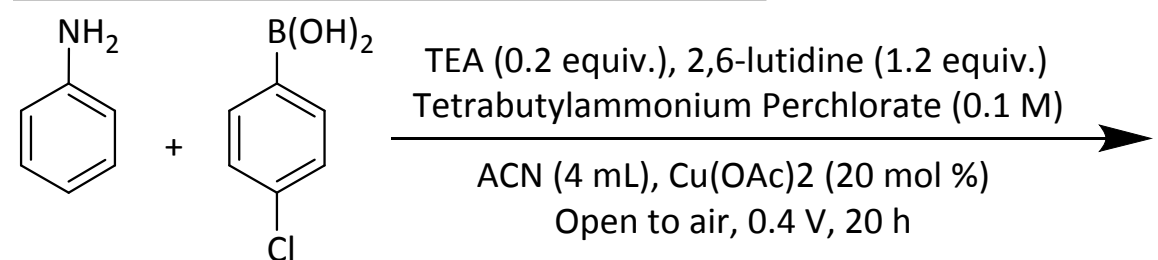

1a<smiles>Clc1ccc(Nc2ccccc2)cc1</smiles>

$3 a$

4-Chloro- $\mathrm{N}$-phenylaniline (3a) (Scheme 2). ${ }^{1} \mathrm{H}$ NMR $\left(\mathrm{CDCl}_{3}, 300 \mathrm{MHz}\right) \delta$ 7.30-7.25 (m, 2H), 7.22-7.18 (m, $2 \mathrm{H}), 7.05-7.02(\mathrm{~m}, 2 \mathrm{H}), 6.99-6.93(\mathrm{~m}, 3 \mathrm{H}), 5.66$ (brs, $1 \mathrm{H}) ;{ }^{13} \mathrm{C} \mathrm{NMR}\left(\mathrm{CDCl}_{3}, 75 \mathrm{MHz}\right) \delta 142.6,141.8,129.4$, 129.2, 125.4, 121.5, 118.8, 118.0. ESI-HRMS (m/z) calcd. for $\mathrm{C}_{12} \mathrm{H}_{10} \mathrm{CIN}[(\mathrm{M}+\mathrm{H})]^{+}:$204.058, found: 204.05751. This is a known compound and the spectral data are identical to those reported in the literature. $^{3}$

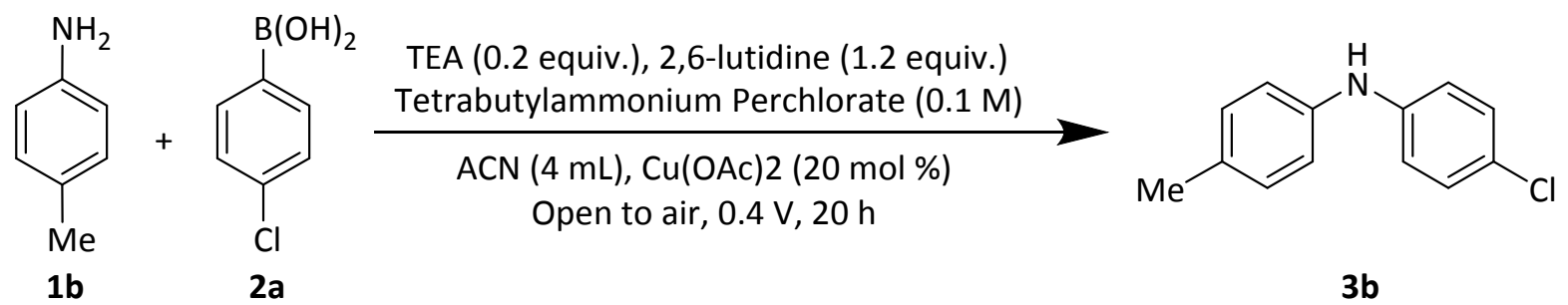

4-Chloro- $\boldsymbol{N}$-(p-tolyl)aniline (3b) (Scheme 2). Following the above general procedure with $\mathbf{1 b}$ (59 $\mathrm{mg}$, $0.55 \mathrm{mmol})$ and $2 \mathrm{a}(112 \mathrm{mg}, 0.71 \mathrm{mmol})$. The crude reaction mixture was purified by flash 
chromatography on a $12 \mathrm{~g}$ silica column (0-40\% EtOAc/Heptane) to provide $\mathbf{3 b}$ (115 $\mathrm{mg}, 0.53 \mathrm{mmol}$, 97\%) as a yellow solid. ${ }^{1} \mathrm{H}$ NMR $\left(\mathrm{CDCl}_{3}, 300 \mathrm{MHz}\right) \delta 7.20-7.16(\mathrm{~m}, 2 \mathrm{H}), 7.10(\mathrm{~d}, J=8.5 \mathrm{~Hz}, 2 \mathrm{H}), 6.97(\mathrm{~d}, J=$ $7.9 \mathrm{~Hz}, 2 \mathrm{H}), 6.91(\mathrm{~d}, \mathrm{~J}=9.1 \mathrm{~Hz}, 2 \mathrm{H}), 5.58$ (brs, $1 \mathrm{H}), 2.32(\mathrm{~s}, 3 \mathrm{H}) ;{ }^{13} \mathrm{C} \mathrm{NMR}\left(\mathrm{CDCl}_{3}, 75 \mathrm{MHz}\right) \delta$ 142.6, 139.7, $131.5,129.9,129.2,124.7,119.2,117.8,20.7$; ESI-HRMS (m/z) calcd. for $\mathrm{C}_{13} \mathrm{H}_{12} \mathrm{CIN}[(\mathrm{M}+\mathrm{H})]^{+}:$: 218.07365, found: 218.07312 . This is a known compound and the spectral data are identical to those reported in the literature. ${ }^{4}$

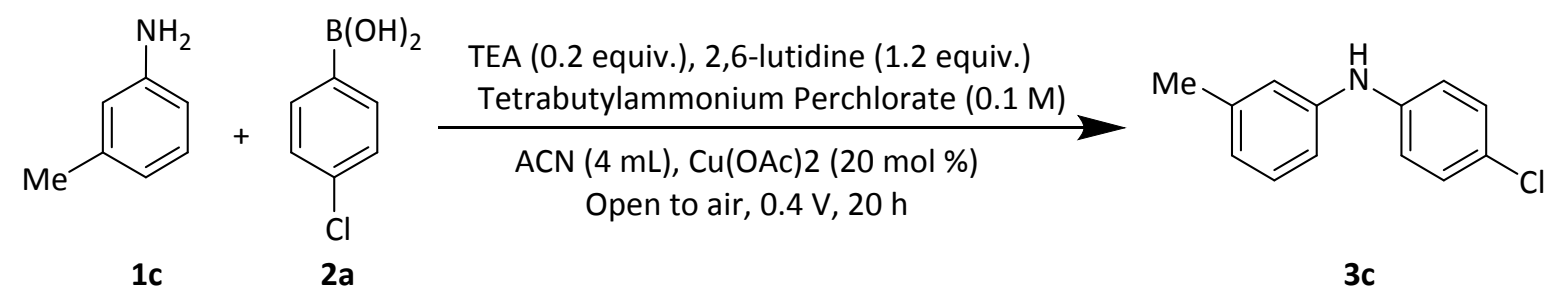

$\mathbf{N}$-(4-Chlorophenyl)-3-methylaniline (3c) (Scheme 2). Following the above general procedure with 1c $(60 \mu \mathrm{L}, 0.55 \mathrm{mmol})$ and $2 \mathrm{a}(112 \mathrm{mg}, 0.71 \mathrm{mmol})$. The crude reaction mixture was purified by flash chromatography on a $12 \mathrm{~g}$ silica column (0-40\% EtOAc/Heptane) to provide 3c (113 mg, $0.52 \mathrm{mmol}$, 94\%) as a waxy orange solid. ${ }^{1} \mathrm{H}$ NMR $\left(\mathrm{CDCl}_{3}, 300 \mathrm{MHz}\right) \delta$ 7.23-7.15 $(\mathrm{m}, 3 \mathrm{H}), 6.99-6.95(\mathrm{~m}, 2 \mathrm{H}), 6.87-6.84$ $(\mathrm{m}, 2 \mathrm{H}), 6.79(\mathrm{~d}, J=7.4 \mathrm{~Hz}, 1 \mathrm{H}), 5.62$ (brs, $1 \mathrm{H}), 2.32(\mathrm{~s}, 3 \mathrm{H}) ;{ }^{13} \mathrm{C} \mathrm{NMR}\left(\mathrm{CDCl}_{3}, 75 \mathrm{MHz}\right) \delta 142.7,142.1$, $139.5,129.4,129.4,125.5,122.5,118.9,115.4,21.7$.; ESI-HRMS (m/z) calcd. for $\mathrm{C}_{13} \mathrm{H}_{12} \mathrm{CIN}[(\mathrm{M}+\mathrm{H})]^{+}$: 218.07365, found: 218.07321 . This is a known compound and the spectral data are identical to those reported in the literature. ${ }^{12}$<smiles>Cc1ccccc1N</smiles>

1d

$2 a$

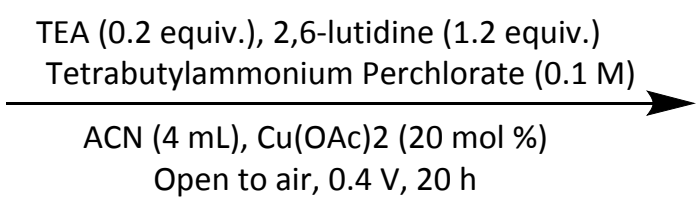<smiles>Cc1ccccc1Nc1ccc(Cl)cc1</smiles>

3d

$\mathbf{N}$-(4-Chlorophenyl)-2-methylaniline (3d) (Scheme 2). Following the above general procedure with $\mathbf{1 d}$ $(60 \mu \mathrm{L}, 0.55 \mathrm{mmol})$ and $2 \mathrm{a}(112 \mathrm{mg}, 0.71 \mathrm{mmol})$. The crude reaction mixture was purified by flash chromatography on a $12 \mathrm{~g}$ silica column (0-40\% EtOAc/Heptane) to provide $3 \mathrm{~d}$ (101 $\mathrm{mg}, 0.46 \mathrm{mmol}$, 84\%) as a brown/orange oil. ${ }^{1} \mathrm{H}$ NMR $\left(\mathrm{CDCl}_{3}, 300 \mathrm{MHz}\right) \delta$ 7.23-7.13 $(\mathrm{m}, 5 \mathrm{H}), 7.00-6.95(\mathrm{~m}, 1 \mathrm{H}), 6.85(\mathrm{~d}, \mathrm{~J}=$

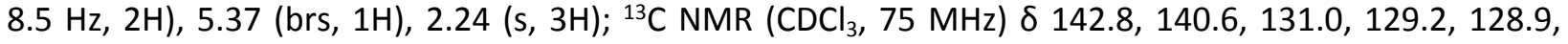
$126.8,124.8,122.6,119.4,118.2,17.8$; ESI-HRMS (m/z) calcd. for $\mathrm{C}_{13} \mathrm{H}_{12} \mathrm{CIN}[(\mathrm{M}+\mathrm{H})]^{+}: 218.07365$, found: 218.07314. This is a known compound and the spectral data are identical to those reported in the literature. ${ }^{12}$

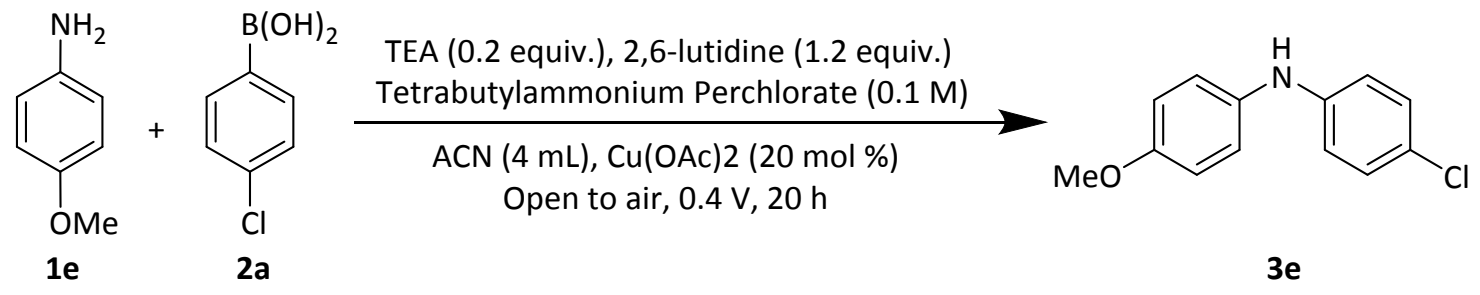

4-Chloro- $\mathbf{N}$-(4-methoxyphenyl)aniline (3e) (Scheme 2). Following the above general procedure with 1e (68 $\mathrm{mg}, 0.55 \mathrm{mmol})$ and $2 \mathrm{a}(112 \mathrm{mg}, 0.71 \mathrm{mmol})$. The crude reaction mixture was purified by flash chromatography on a $12 \mathrm{~g}$ silica column (0-40\% EtOAc/Heptane) to provide $3 \mathrm{e}$ (75 mg, $0.32 \mathrm{mmol}, 69 \%)$ as a waxy yellow solid. ${ }^{1} \mathrm{H}$ NMR $\left(\mathrm{CDCl}_{3}, 300 \mathrm{MHz}\right) \delta$ 7.16-7.12 $(\mathrm{m}, 2 \mathrm{H}), 7.05-7.02(\mathrm{~m}, 2 \mathrm{H}), 6.88-6.85(\mathrm{~m}$, 2H), 6.81-6.78 (m, 2H), 5.47 (brs, 1H), $\left.3.79(\mathrm{~s}, 3 \mathrm{H}) ;{ }^{13} \mathrm{C} \mathrm{NMR} \mathrm{(CDCl}, 75 \mathrm{MHz}\right) \delta 155.6,143.9,135.2,129.1$, 123.9, 122.5, 116.6, 114.7, 55.5. ESI-HRMS $(\mathrm{m} / \mathrm{z})$ calcd. for $\mathrm{C}_{13} \mathrm{H}_{12} \mathrm{CINO}[(\mathrm{M}+\mathrm{H})]^{+}:$234.06857, found: 
234.06807. This is a known compound and the spectral data are identical to those reported in the literature. ${ }^{5}$

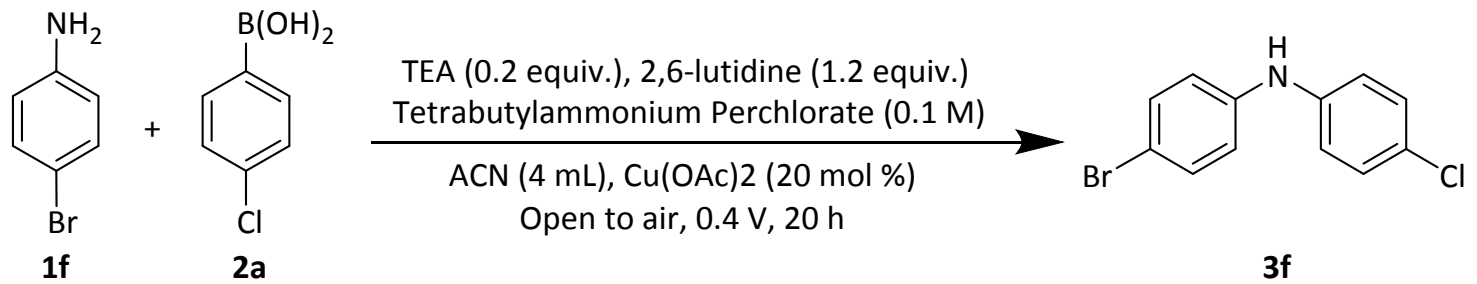

4-Bromo-N-(4-chlorophenyl)aniline (3f) (Scheme 2). Following the above general procedure with $1 \mathbf{f}$ (94 $\mathrm{mg}, 0.55 \mathrm{mmol})$ and $2 \mathrm{a}(112 \mathrm{mg}, 0.71 \mathrm{mmol})$. The crude reaction mixture was purified by flash chromatography on a $12 \mathrm{~g}$ silica column (0-40\% EtOAc/Heptane) to provide $\mathbf{3 f}$ (125 $\mathrm{mg}, 0.44 \mathrm{mmol}, \mathbf{8 1 \%})$ as a light brown/orange solid. ${ }^{1} \mathrm{H}$ NMR $\left(\mathrm{CDCl}_{3}, 300 \mathrm{MHz}\right) \delta$ 7.38-7.32 $(\mathrm{m}, 2 \mathrm{H}), 7.23-7.19(\mathrm{~m}, 2 \mathrm{H}), 6.98-$ $6.93(\mathrm{~m}, 2 \mathrm{H}), 6.91-6.87(\mathrm{~m}, 2 \mathrm{H}), 5.63$ (brs, $1 \mathrm{H}) ;{ }^{13} \mathrm{C} \mathrm{NMR}\left(\mathrm{CDCl}_{3}, 75 \mathrm{MHz}\right) \delta$ 141.9, 141.1, 132.2, 129.4, 126.2, 119.3, 113.2; ESI-HRMS (m/z) calcd. for $\mathrm{C}_{12} \mathrm{H}_{9} \mathrm{BrCIN}[(\mathrm{M}+\mathrm{H})]^{+} 281.96851$, found: 281.96752 . This is a known compound and the spectral data are identical to those reported in the literature. ${ }^{12}$<smiles>Nc1ccc(Cl)cc1</smiles>

$1 \mathrm{~g}$

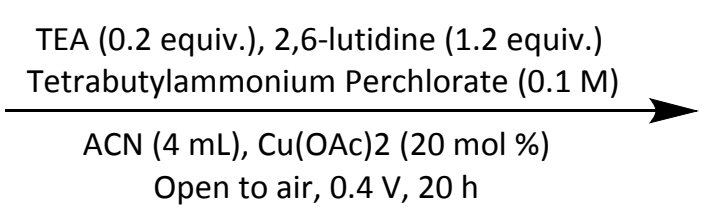

Open to air, $0.4 \mathrm{~V}, 20 \mathrm{~h}$<smiles>Clc1ccc(Nc2ccc(Cl)cc2)cc1</smiles>

$3 g$

Bis(4-chlorophenyl)amine (3g) (Scheme 2). Following the above general procedure with $\mathbf{1 g}(89 \mathrm{mg}, 0.55$ $\mathrm{mmol}$ ) and $2 \mathrm{a}(112 \mathrm{mg}, 0.71 \mathrm{mmol})$. The crude reaction mixture was purified by flash chromatography on a $12 \mathrm{~g}$ silica column (0-40\% EtOAc/Heptane) to provide $\mathbf{3 g}$ (67 mg, $0.25 \mathrm{mmol}, \mathbf{4 5 \%}$ ) as a light brown

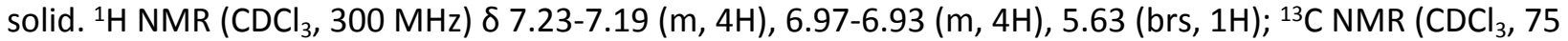
$\mathrm{MHz}) \delta 141.3,129.3,126.0,119.0$. ESI-HRMS (m/z) calcd. for $\mathrm{C}_{12} \mathrm{H}_{9} \mathrm{Cl}_{2} \mathrm{~N}[(\mathrm{M}+\mathrm{H})]^{+}:$238.01903, found: 238.01857. This is a known compound and the spectral data are identical to those reported in the literature. ${ }^{6}$<smiles>N#Cc1ccc(Br)cc1</smiles>

1h

$2 a$

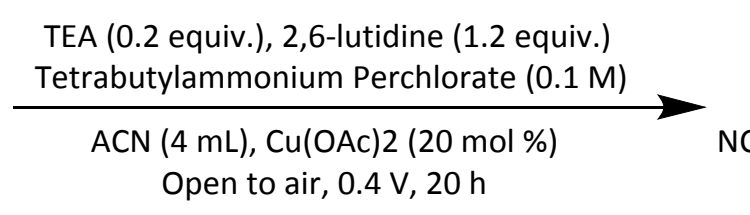

Open to air, $0.4 \mathrm{~V}, 20 \mathrm{~h}$<smiles>Clc1ccc(Nc2ccc(Cl)cc2)cc1</smiles>

3h

4-((4-Chlorophenyl)amino)benzonitrile ( $3 \mathrm{~h})$ (Scheme 2). Following the above general procedure with $\mathbf{1 h}$ $(65 \mathrm{mg}, 0.55 \mathrm{mmol})$ and $2 \mathrm{a}(112 \mathrm{mg}, 0.71 \mathrm{mmol})$. The crude reaction mixture was purified by flash chromatography on a $12 \mathrm{~g}$ silica column (0-40\% EtOAc/Heptane) to provide $\mathbf{3 h}$ ( $50 \mathrm{mg}, 0.22 \mathrm{mmol}, 40 \%)$ as a_white solid. ${ }^{1} \mathrm{H}$ NMR $\left(\mathrm{CDCl}_{3}, 300 \mathrm{MHz}\right) \delta$ 7.49-7.46 $(\mathrm{m}, 2 \mathrm{H}), 7.32-7.28(\mathrm{~m}, 2 \mathrm{H}), 7.13-7.08(\mathrm{~m}, 2 \mathrm{H})$, 6.98-6.92 (m, 2H), 6.12 (brs, $\left.1 \mathrm{H}) ;{ }^{13} \mathrm{C} \mathrm{NMR} \mathrm{(CDCl}, 75 \mathrm{MHz}\right) \delta 147.6,138.6,133.8,129.6,128.8,122.3$, 119.7, 115.1, 101.9; ESI-HRMS (m/z) calcd. for $\mathrm{C}_{13} \mathrm{H}_{9} \mathrm{ClN}_{2}[(\mathrm{M}+\mathrm{H})]^{+}: 229.05325$, found: 229.05281 . This is a known compound and the spectral data are identical to those reported in the literature. ${ }^{12}$ 
<smiles>CCC(=O)c1ccc(N)cc1</smiles>

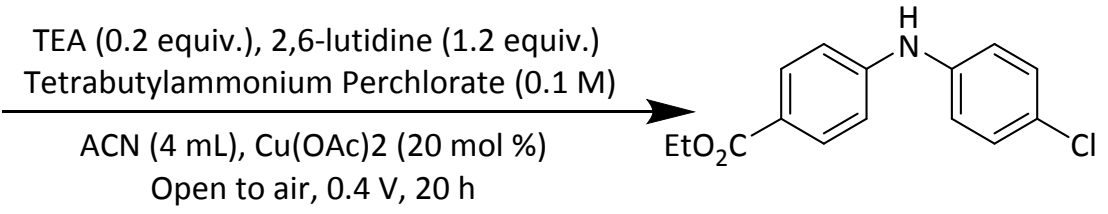

$1 \mathrm{i}$

2a

$3 \mathbf{i}$

Ethyl 4-((4-chlorophenyl)amino)benzoate (3i) (Scheme 2). Following the above general procedure with 1i $(91 \mathrm{mg}, 0.55 \mathrm{mmol}$ ) and $2 \mathrm{a}$ (112 $\mathrm{mg}, 0.71 \mathrm{mmol})$. The crude reaction mixture was purified by flash chromatography on a $12 \mathrm{~g}$ silica column (0-40\% EtOAc/Heptane) to provide $\mathbf{3 i}$ (70 $\mathrm{mg}, 0.25 \mathrm{mmol}, \mathbf{4 6 \%})$ as a white solid. ${ }^{1} \mathrm{H}$ NMR $\left(\mathrm{CDCl}_{3}, 300 \mathrm{MHz}\right) \delta$ 7.90-7.84 $(\mathrm{m}, 2 \mathrm{H}), 7.25-7.20(\mathrm{~m}, 2 \mathrm{H}), 7.06-7.00(\mathrm{~m}, 2 \mathrm{H})$, 6.93-6.88 (m, 2H), $5.96(\mathrm{brs}, 1 \mathrm{H}), 4.26(\mathrm{q}, J=7.0 \mathrm{~Hz}, 2 \mathrm{H}), 1.30(\mathrm{t}, J=7.0 \mathrm{~Hz}, 3 \mathrm{H}) ;{ }^{13} \mathrm{C} \mathrm{NMR}\left(\mathrm{CDCl}_{3}, 75 \mathrm{MHz}\right)$ $\delta 166.4,147.4,139.6,131.4,129.5,127.8,122.0,121.4,114.8,60.5,14.4 ;$ ESI-HRMS (m/z) calcd. for $\mathrm{C}_{15} \mathrm{H}_{14} \mathrm{CINO}_{2}[(\mathrm{M}+\mathrm{H})]^{+}: 276.07913$, found: 276.07864 . This is a known compound and the spectral data are identical to those reported in the literature. ${ }^{12}$<smiles>CC(=O)c1ccc(Cl)cc1</smiles>

$1 \mathbf{j}$

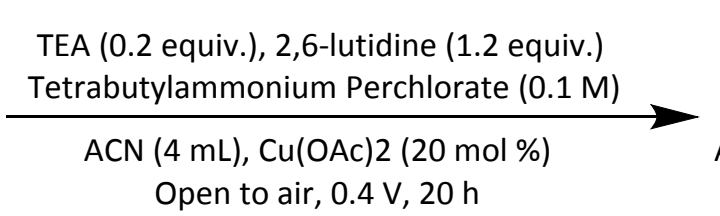

Open to air, $0.4 \mathrm{~V}, 20 \mathrm{~h}$<smiles>Clc1ccc(Nc2ccc(Cl)cc2)cc1</smiles>

3j

1-(4-((4-Chlorophenyl)amino)phenyl)ethan-1-one (3j) (Scheme 2). Following the above general procedure with $1 \mathbf{j}(74 \mathrm{mg}, 0.55 \mathrm{mmol})$ and $2 \mathrm{a}(112 \mathrm{mg}, 0.71 \mathrm{mmol})$. The crude reaction mixture was purified by flash chromatography on a $12 \mathrm{~g}$ silica column (0-40\% EtOAc/Heptane) to provide $\mathbf{3 j}$ (65 $\mathrm{mg}$, $0.27 \mathrm{mmol}, 48 \%)$ as a white solid. ${ }^{1} \mathrm{H}$ NMR $\left(\mathrm{CDCl}_{3}, 300 \mathrm{MHz}\right) \delta$ 7.81-7.78 $(\mathrm{m}, 2 \mathrm{H}), 7.23-7.20(\mathrm{~m}, 2 \mathrm{H}), 7.05-$ $7.02(\mathrm{~m}, 2 \mathrm{H}), 6.91-6.88(\mathrm{~m}, 2 \mathrm{H}), 6.11(\mathrm{brs}, 1 \mathrm{H}), 2.46(\mathrm{~s}, 3 \mathrm{H}) ;{ }^{13} \mathrm{C} \mathrm{NMR}\left(\mathrm{CDCl}_{3}, 75 \mathrm{MHz}\right) \delta 196.4,147.9$, $139.3,130.6,129.5,129.4,128.1,121.7,114.6,26.2$; ESI-HRMS (m/z) calcd. for $\mathrm{C}_{14} \mathrm{H}_{12} \mathrm{CINO}[(\mathrm{M}+\mathrm{H})]^{+}$: 246.06857, found: 246.06822 . This is a known compound and the spectral data are identical to those reported in the literature. ${ }^{12}$<smiles>Nc1ccc(C(F)(F)F)cc1</smiles>

$1 \mathrm{k}$<smiles>[OH2+]c1ccc(Cl)cc1</smiles>

2a

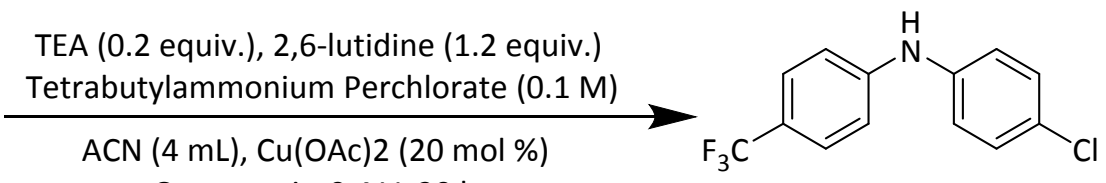

Open to air, $0.4 \mathrm{~V}, 20 \mathrm{~h}$

4-Chloro-N-(4-(trifluoromethyl)phenyl)aniline (3k) (Scheme 2). Following the above general procedure with $1 \mathbf{k}(69 \mu \mathrm{L}, 0.55 \mathrm{mmol})$ and $2 \mathrm{a}(112 \mathrm{mg}, 0.71 \mathrm{mmol})$. The crude reaction mixture was purified by flash chromatography on a $12 \mathrm{~g}$ silica column (0-40\% EtOAc/Heptane) to provide $3 \mathbf{k}$ (98 $\mathrm{mg}, 0.36 \mathrm{mmol}$, 66\%) as a brown oil. ${ }^{1} \mathrm{H}$ NMR $\left(\mathrm{CDCl}_{3}, 300 \mathrm{MHz}\right) \delta 7.40(\mathrm{~d}, J=8.5 \mathrm{~Hz}, 2 \mathrm{H}), 7.20(\mathrm{~d}, J=8.5 \mathrm{~Hz}, 2 \mathrm{H}), 7.01-6.93$ $(\mathrm{m}, 4 \mathrm{H}), 5.76$ (brs, $1 \mathrm{H}) ;{ }^{13} \mathrm{C} \mathrm{NMR}\left(\mathrm{CDCl}_{3}, 75 \mathrm{MHz}\right) \delta 146.3,139.8,129.5,127.7,126.6,124.5,122.9,122.1$, 121.1, 115.6; ESI-HRMS (m/z) calcd. for $\mathrm{C}_{13} \mathrm{H}_{9} \mathrm{ClF}_{3} \mathrm{~N}[(\mathrm{M}+\mathrm{H})]^{+}: 272.04484$, found: 272.04511 . This is a known compound and the spectral data are identical to those reported in the literature. ${ }^{4}$ 
<smiles>Nc1cc(Cl)cc(Cl)c1</smiles>

1l

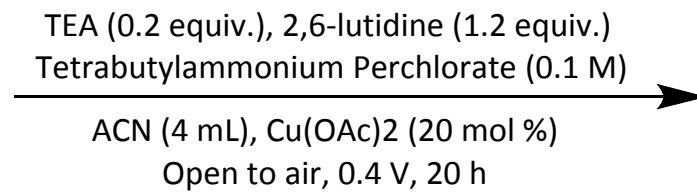

Open to air, $0.4 \mathrm{~V}, 20 \mathrm{~h}$<smiles>Clc1ccc(Nc2cc(Cl)cc(Cl)c2)cc1</smiles>

31

3,5-Dichloro- $\mathbf{N}$-(4-chlorophenyl)aniline (3I) (Scheme 2). Following the above general procedure with $\mathbf{1 l}$ ( $89 \mathrm{mg}, 0.55 \mathrm{mmol})$ and $2 \mathrm{a}(112 \mathrm{mg}, 0.71 \mathrm{mmol})$. The crude reaction mixture was purified by flash chromatography on a $12 \mathrm{~g}$ silica column (0-40\% EtOAc/Heptane) to provide $\mathbf{3 l}$ (67 mg, $0.25 \mathrm{mmol}$, 45\%) as a white solid. ${ }^{1} \mathrm{H}$ NMR $\left(300 \mathrm{MHz}, \mathrm{CDCl}_{3}\right) \delta 7.28-7.21(\mathrm{~m}, 2 \mathrm{H}), 7.03-6.95(\mathrm{~m}, 2 \mathrm{H}), 6.81(\mathrm{dd}, \mathrm{J}=8.5$, $1.8 \mathrm{~Hz}, 3 \mathrm{H}), 5.67$ (brs, $1 \mathrm{H}) .{ }^{13} \mathrm{C}$ NMR $\left(75 \mathrm{MHz}, \mathrm{CDCl}_{3}\right) \delta 145.4,139.6,135.7,129.7,128.0,121.3,120.4$, 114.5. ESI-HRMS (m/z) calcd. for $\mathrm{C}_{12} \mathrm{H}_{8} \mathrm{Cl}_{3} \mathrm{~N}[(\mathrm{M}+\mathrm{H})]^{+}: 271.97951$, found: 271.97924.

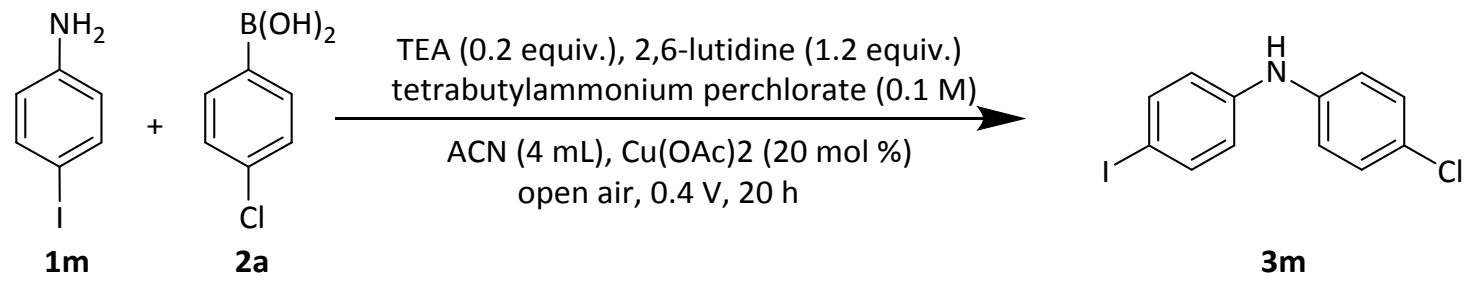

4-chloro- $\mathbf{N}$-(4-iodophenyl)aniline $\mathbf{( 3 m )}$ (Scheme 2). Following the above general procedure with $\mathbf{1 m}$ $(120 \mathrm{mg}, 0.55 \mathrm{mmol})$ and $2 \mathrm{a}(112 \mathrm{mg}, 0.71 \mathrm{mmol})$. The crude reaction mixture was purified by flash

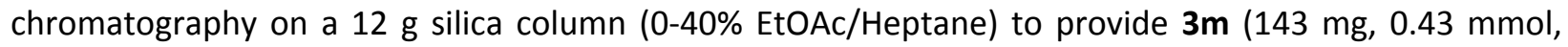
79\%) as a waxy red solid. ${ }^{1} \mathrm{H}$ NMR $\left(\mathrm{CDCl}_{3}, 300 \mathrm{MHz}\right) \delta$ 7.55-7.51 $(\mathrm{m}, 2 \mathrm{H}), 7.24-7.20(\mathrm{~m}, 2 \mathrm{H}), 7.00-6.95(\mathrm{~m}$, $2 \mathrm{H}), 6.82-6.78(\mathrm{~m}, 2 \mathrm{H}), 5.65$ (brs, $1 \mathrm{H}) ;{ }^{13} \mathrm{C} \mathrm{NMR}\left(\mathrm{CDCl}_{3}, 75 \mathrm{MHz}\right) \delta 142.7,141.0,138.2,129.4,126.4$, 119.5, 82.8; DART-HRMS (m/z) calcd. for $\mathrm{C}_{12} \mathrm{H}_{9} \mathrm{ClIN}[(\mathrm{M}+\mathrm{H})+]$ : 329.95465 , found: 329.9541.

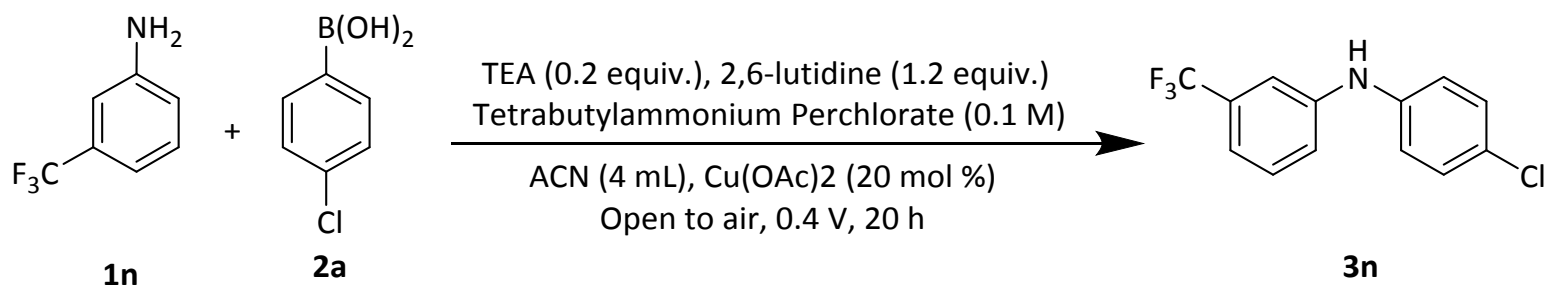

4-Chloro- $\mathbf{N}$-(4-(trifluoromethyl)phenyl)aniline (3n) (Scheme 2). Following the above general procedure with $1 \mathrm{n}(69 \mu \mathrm{L}, 0.55 \mathrm{mmol})$ and $2 \mathrm{a}(112 \mathrm{mg}, 0.71 \mathrm{mmol})$. The crude reaction mixture was purified by flash chromatography on a $12 \mathrm{~g}$ silica column (0-40\% EtOAc/Heptane) to provide $3 \mathrm{n}$ (75 mg, $0.28 \mathrm{mmol}$, 50\%) as a brown oil. ${ }^{1} \mathrm{H}$ NMR (300 MHz, Chloroform-d) $\delta 7.41-7.31(\mathrm{~m}, 1 \mathrm{H}), 7.30-7.22(\mathrm{~m}, 3 \mathrm{H}), 7.23-$ $7.12(\mathrm{~m}, 2 \mathrm{H}), 7.07-6.99(\mathrm{~m}, 2 \mathrm{H}), 5.79(\mathrm{~s}, 1 \mathrm{H}) ;{ }^{13} \mathrm{C}$ NMR (75 MHz, Chloroform-d) $\delta$ 143.6, 140.5, 132.1, 131.7, 130.0, 129.6, 127.1, 120.1, 119.98, 117.4, 113.5.; ESI-HRMS (m/z) calcd. for $\mathrm{C}_{13} \mathrm{H}_{9} \mathrm{ClF}_{3} \mathrm{~N}[(\mathrm{M}+\mathrm{H})]^{+}$: 272.04484, found: 272.04402 . 


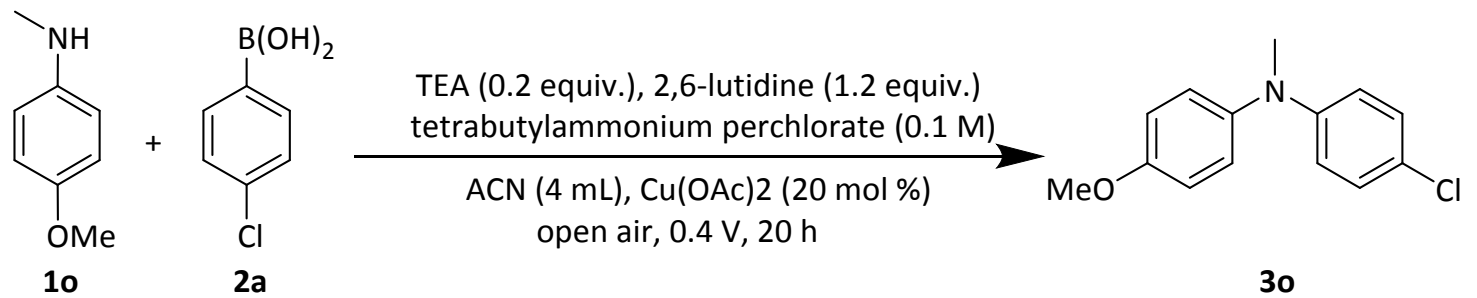

4-chloro-N-(4-methoxyphenyl)-N-methylaniline (30) (Scheme 2). Following the above general procedure with $10(75 \mathrm{mg}, 0.55 \mathrm{mmol})$ and $2 \mathrm{a}(112 \mathrm{mg}, 0.71 \mathrm{mmol})$. The crude reaction mixture was purified by flash chromatography on a $12 \mathrm{~g}$ silica column (0-40\% EtOAc/Heptane) to provide 30 (52 $\mathrm{mg}$, $0.20 \mathrm{mmol}, 39 \%)$ as a yellow solid. ${ }^{1} \mathrm{H}$ NMR $\left(\mathrm{CDCl}_{3}, 300 \mathrm{MHz}\right) \delta 7.12-7.08(\mathrm{~m}, 2 \mathrm{H}), 7.08-7.04(\mathrm{~m}, 2 \mathrm{H}), 6.90-$ $6.86(\mathrm{~m}, 2 \mathrm{H}), 6.68-6.64(\mathrm{~m}, 2 \mathrm{H}), 3.80(\mathrm{~s}, 3 \mathrm{H}), 3.21(\mathrm{~s}, 3 \mathrm{H}) ;{ }^{13} \mathrm{C} \mathrm{NMR}\left(\mathrm{CDCl}_{3}, 75 \mathrm{MHz}\right) \delta 156.7,148.4,141.8$, $128.7,126.6,122.9,116.4,114.9,55.5,40.6$; ESI-HRMS (m/z) calcd. for $\mathrm{C}_{14} \mathrm{H}_{14} \mathrm{CINO}[(\mathrm{M}+\mathrm{H})]^{+}: 248.08422$, found: 248.08359 .<smiles>Nc1ccccc1</smiles>

$1 \mathrm{p}$

2a

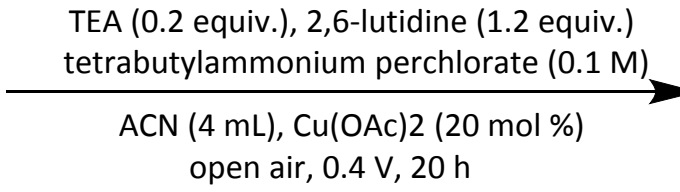<smiles>Clc1ccc(Nc2cccnc2)cc1</smiles>

$3 p$

$\mathbf{N}$-(4-chlorophenyl)pyridin-3-amine (3p) (Scheme 2). Following the above general procedure with 1p (120 mg, $0.55 \mathrm{mmol}$ ) and $2 \mathrm{a}(112 \mathrm{mg}, 0.71 \mathrm{mmol}$ ). The crude reaction mixture was purified by flash chromatography on a $12 \mathrm{~g}$ silica column $(0-10 \% \mathrm{DCM} / \mathrm{MeOH})$ to provide $3 \mathrm{p}(33 \mathrm{mg}, 0.17 \mathrm{mmol}, \mathbf{3 0 \%})$ as a white solid. ${ }^{1} \mathrm{H}$ NMR $(300 \mathrm{MHz}$, Chloroform-d) $\delta 8.39$ (dd, $J=2.8,0.8 \mathrm{~Hz}, 1 \mathrm{H}), 8.21$ (dd, J = 4.7, $1.5 \mathrm{~Hz}$, $1 \mathrm{H}$ ), 7.40 (ddd, $J=8.3,2.8,1.4 \mathrm{~Hz}, 1 \mathrm{H}), 7.31-7.18(\mathrm{~m}, 3 \mathrm{H}), 7.13-6.96(\mathrm{~m}, 2 \mathrm{H}), 5.79(\mathrm{brs}, 1 \mathrm{H}) ;{ }^{13} \mathrm{C}$ NMR (75 MHz, Chloroform-d) $\delta 142.4,140.7,140.4,139.4,129.5,126.7,123.8,123.8,119.4 . ;$ DART-HRMS (m/z) calcd. for $\mathrm{C}_{11} \mathrm{H}_{9} \mathrm{ClN}_{2}[(\mathrm{M}+\mathrm{H})+]$ : 205.05271, found: 205.05333 .

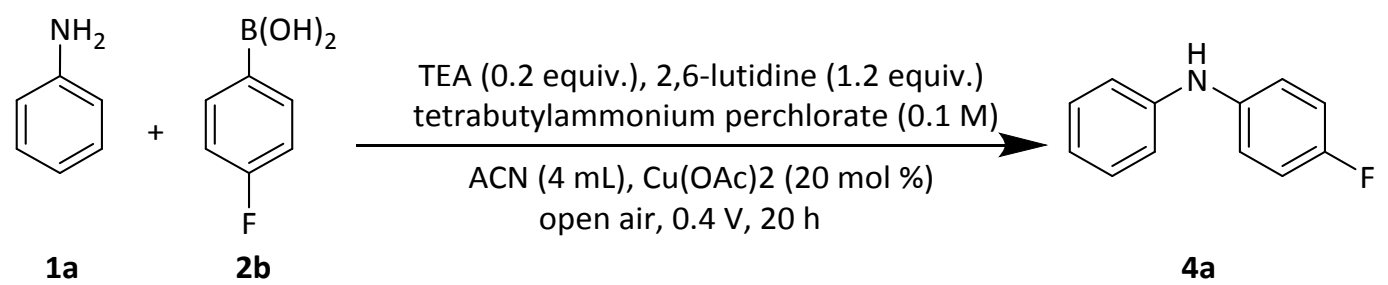

4-Fluoro-N-phenylaniline (4a) (Scheme 3) Following the above general procedure with 1a (50 $\mu \mathrm{L}, 0.55$ $\mathrm{mmol})$ and $\mathbf{2 b}(100 \mathrm{mg}, 0.71 \mathrm{mmol})$. The crude reaction mixture was purified by flash chromatography on a $12 \mathrm{~g}$ silica column (0-40\% EtOAc/Heptane) to provide 4 a (74 $\mathrm{mg}, 0.40 \mathrm{mmol}, \mathbf{7 2} \%)$ as a pale yellow solid. ${ }^{1} \mathrm{H} \mathrm{NMR}\left(\mathrm{CDCl}_{3}, 300 \mathrm{MHz}\right)$ 8 7.19-7.14 $(\mathrm{m}, 2 \mathrm{H}), 6.98-6.94(\mathrm{~m}, 2 \mathrm{H}), 6.92-6.83(\mathrm{~m}, 4 \mathrm{H}), 6.83-6.79(\mathrm{~m}$, $1 \mathrm{H}), 5.50$ (brs, $1 \mathrm{H}) ;{ }^{13} \mathrm{C} \mathrm{NMR}\left(\mathrm{CDCl}_{3}, 75 \mathrm{MHz}\right) \delta 158.0,143.9,138.9,129.4,120.6,120.5,116.7$, 115.9; ESIHRMS (m/z) calcd. for $\mathrm{C}_{12} \mathrm{H}_{10} \mathrm{FN}[(\mathrm{M}+\mathrm{H})]^{+}: 188.08701$, found: 188.08777 . This is a known compound and the spectral data are identical to those reported in the literature. ${ }^{3}$ 


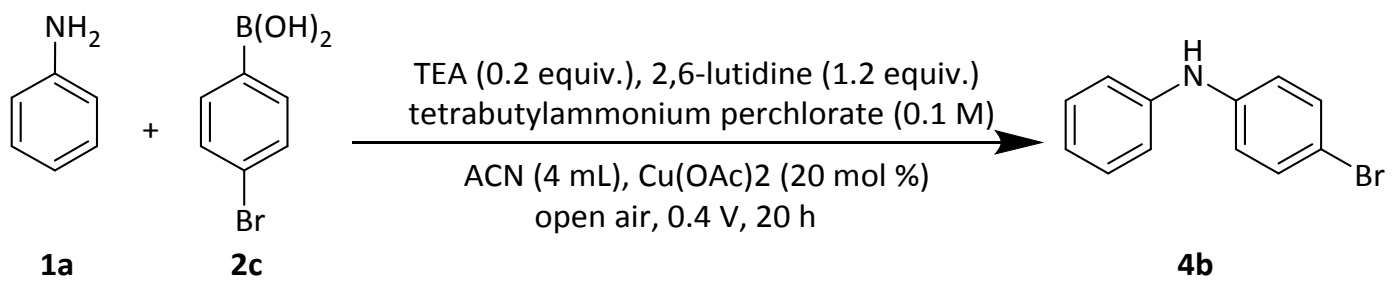

4-Bromo- $\mathbf{N}$-phenylaniline (4b) (Scheme 3). Following the above general procedure with $\mathbf{1 a}$ (50 $\mu \mathrm{L}, 0.55$ $\mathrm{mmol}$ ) and $2 \mathrm{c}$ ( $143 \mathrm{mg}, 0.71 \mathrm{mmol})$. The crude reaction mixture was purified by flash chromatography on a $12 \mathrm{~g}$ silica column (0-40\% EtOAc/Heptane) to provide $\mathbf{4 b}$ (114 $\mathrm{mg}, 0.57 \mathrm{mmol}, \mathbf{8 4 \%})$ as a brown solid. ${ }^{1} \mathrm{H}$ NMR $\left(\mathrm{CDCl}_{3}, 300 \mathrm{MHz}\right) \delta$ 7.29-7.18 (m, 4H), $6.99(\mathrm{dd}, J=8.5,1.1 \mathrm{~Hz}, 2 \mathrm{H}), 6.91-6.85(\mathrm{~m}, 3 \mathrm{H}), 5.60$ (brs, $1 \mathrm{H}) ;{ }^{13} \mathrm{C} \mathrm{NMR}\left(\mathrm{CDCl}_{3}, 75 \mathrm{MHz}\right) \delta 142.4,142.3,132.1,129.4,121.6,119.0,118.2,112.6$. ESI-HRMS (m/z) calcd. for $\mathrm{C}_{12} \mathrm{H}_{10} \mathrm{BrN}$ [(M+H) $]^{+}: 248.00748$, found: 248.00703 . This is a known compound and the spectral data are identical to those reported in the literature. ${ }^{3}$

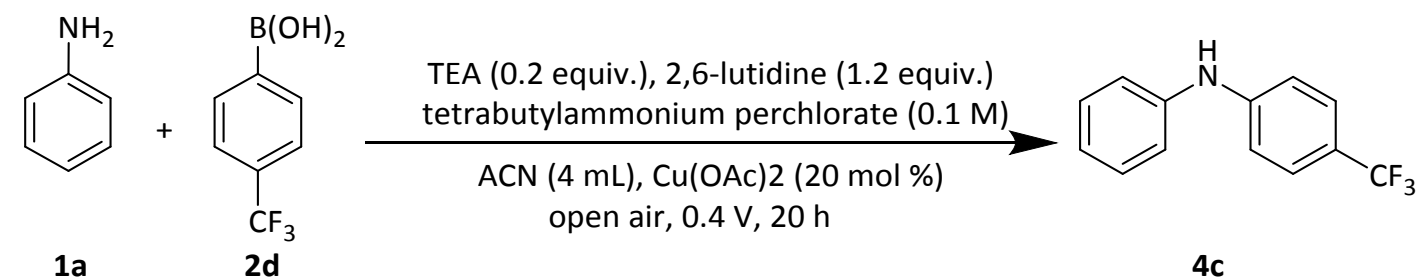

$\mathbf{N}$-Phenyl-4-(trifluoromethyl)aniline (4c) (Scheme 3). Following the above general procedure with 1a $(50 \mu \mathrm{L}, 0.55 \mathrm{mmol})$ and $\mathbf{2 d}(136 \mathrm{mg}, 0.71 \mathrm{mmol})$. The crude reaction mixture was purified by flash chromatography on a $12 \mathrm{~g}$ silica column (0-40\% EtOAc/Heptane) to provide 4c (109 $\mathrm{mg}, 0.46 \mathrm{mmol}$, 83\%) as a brown solid. ${ }^{1} \mathrm{H}$ NMR $\left(\mathrm{CDCl}_{3}, 300 \mathrm{MHz}\right) \delta 7.39(\mathrm{~d}, J=5.1 \mathrm{~Hz}, 2 \mathrm{H}), 7.29-7.24(\mathrm{~m}, 2 \mathrm{H}), 7.10-7.07$ $(\mathrm{m}, 2 \mathrm{H}), 7.01-6.96(\mathrm{~m}, 3 \mathrm{H}), 5.85$ (brs, $1 \mathrm{H}) ;{ }^{13} \mathrm{C}$ NMR (101 MHz, Chloroform-d) $\delta$ 146.4, 139.9, 129.6, 127.7, 126.8, 125.9, 122.1, 121.1, 115.6. ESI-HRMS (m/z) calcd. for $\mathrm{C}_{13} \mathrm{H}_{10} \mathrm{~F}_{3} \mathrm{~N}[(\mathrm{M}+\mathrm{H})]^{+}:$238.08436, found: 238.08379 . This is a known compound and the spectral data are identical to those reported in the literature. $^{7}$

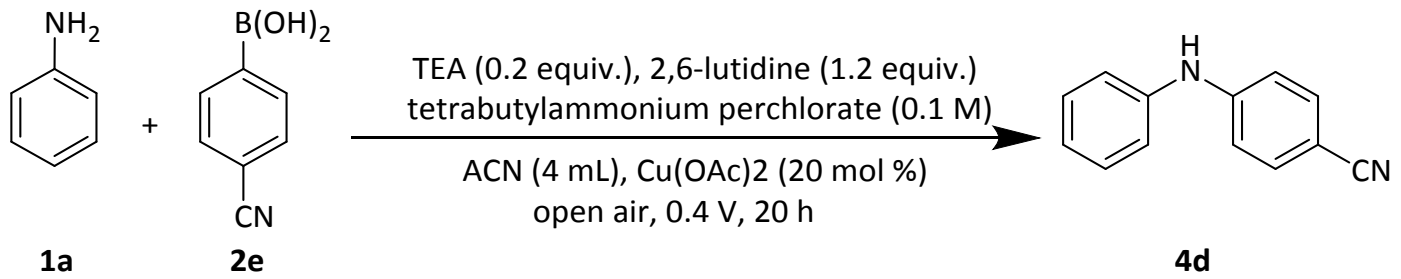

4-(Phenylamino)benzonitrile (4d) (Scheme 3). Following the above general procedure with 1 a $(50 \mu \mathrm{L}$, $0.55 \mathrm{mmol}$ ) and $2 \mathrm{e}(105 \mathrm{mg}, 0.71 \mathrm{mmol})$. The crude reaction mixture was purified by flash chromatography on a $12 \mathrm{~g}$ silica column (0-40\% EtOAc/Heptane) to provide $\mathbf{4 d}$ ( $90 \mathrm{mg}, 0.46 \mathrm{mmol}, \mathbf{8 4 \%})$ as a white solid. ${ }^{1} \mathrm{H}$ NMR $\left(\mathrm{CDCl}_{3}, 300 \mathrm{MHz}\right) \delta$ 7.43-7.39 $(\mathrm{m}, 2 \mathrm{H}), 7.32-7.27(\mathrm{~m}, 2 \mathrm{H}), 7.12-7.03(\mathrm{~m}, 3 \mathrm{H})$, 6.92-6.89 (m, 2H), 6.04 (brs, $1 \mathrm{H}) ;{ }^{13} \mathrm{C} \mathrm{NMR}\left(\mathrm{CDCl}_{3}, 75 \mathrm{MHz}\right) \delta 148.0,139.9,133.8,129.6,124.0,121.2$, $119.9,114.9,101.5$. ESI-HRMS (m/z) calcd. for $\mathrm{C}_{13} \mathrm{H}_{10} \mathrm{~N}_{2}[(\mathrm{M}+\mathrm{H})]^{+}: 195.09222$, found: 195.09286. This is a known compound and the spectral data are identical to those reported in the literature. ${ }^{8}$ 


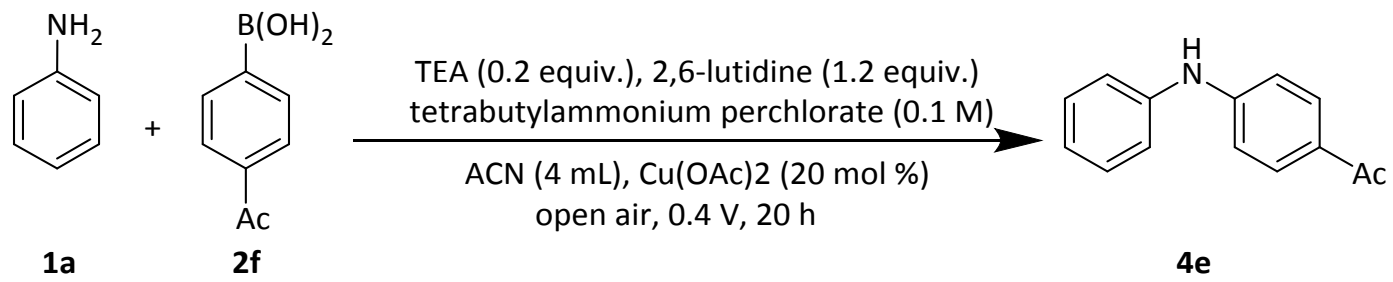

1-(4-(Phenylamino)phenyl)ethanone (4e) (Scheme 3). Following the above general procedure with 1a $(50 \mu \mathrm{L}, 0.55 \mathrm{mmol})$ and $2 \mathrm{f}(117 \mathrm{mg}, 0.71 \mathrm{mmol})$. The crude reaction mixture was purified by flash chromatography on a $12 \mathrm{~g}$ silica column (0-40\% EtOAc/Heptane) to provide $4 \mathrm{e}$ (75 $\mathrm{mg}, 0.36 \mathrm{mmol}, 65 \%)$ as a waxy yellow solid ${ }^{1} \mathrm{H}$ NMR $\left(\mathrm{CDCl}_{3}, 300 \mathrm{MHz}\right) \delta 7.81-7.77(\mathrm{~m}, 2 \mathrm{H}), 7.27(\mathrm{q}, J=5.3 \mathrm{~Hz}, 2 \mathrm{H}), 7.11(\mathrm{~d}, J=$ $7.4 \mathrm{~Hz}, 2 \mathrm{H}), 7.00(\mathrm{t}, J=7.4 \mathrm{~Hz}, 1 \mathrm{H}), 6.93-6.90(\mathrm{~m}, 2 \mathrm{H}), 6.12(\mathrm{brs}, 1 \mathrm{H}), 2.45(\mathrm{~s}, 3 \mathrm{H}) ;{ }^{13} \mathrm{C} \mathrm{NMR}\left(\mathrm{CDCl}_{3}, 75\right.$ $\mathrm{MHz}) \delta 196.4,148.3,140.6,130.6,129.5,129.0,123.4,120.7,114.4,26.2$; ESI-HRMS (m/z) calcd. for $\mathrm{C}_{14} \mathrm{H}_{13} \mathrm{NO}[(\mathrm{M}+\mathrm{H})]^{+}: 212.10754$, found: 212.10699 . This is a known compound and the spectral data are identical to those reported in the literature. ${ }^{9}$

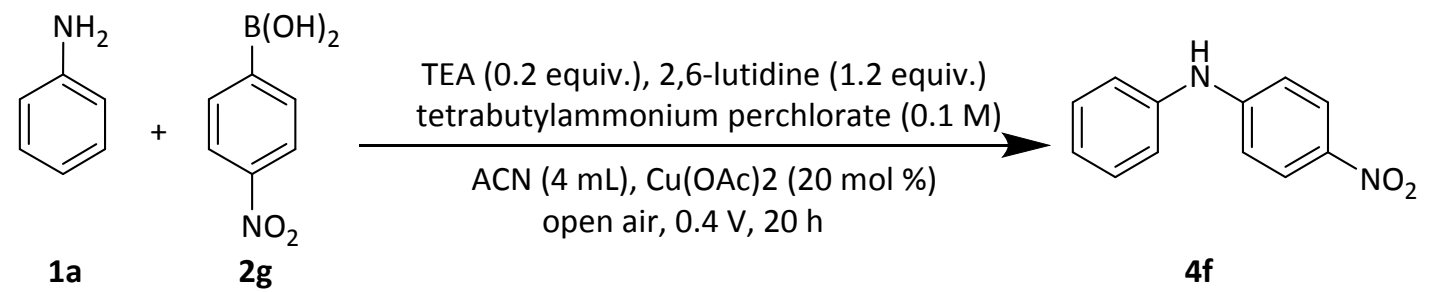

4-nitro-N-phenyl-aniline (4f) (Scheme 3). Following the above general procedure with 1a (50 $\mu \mathrm{L}, 0.55$ $\mathrm{mmol}$ ) and $\mathbf{2 g}(119 \mathrm{mg}, 0.71 \mathrm{mmol})$. The crude reaction mixture was purified by flash chromatography on a $12 \mathrm{~g}$ silica column (0-40\% EtOAc/Heptane) to provide $\mathbf{4 f}$ ( $75 \mathrm{mg}, 0.35 \mathrm{mmol}, \mathbf{6 4 \%}$ ) as a yellow solid. ${ }^{1} \mathrm{H}$ NMR $\left(\mathrm{CDCl}_{3}, 300 \mathrm{MHz}\right) \delta 8.12-8.07(\mathrm{~m}, 2 \mathrm{H}), 7.40-7.34(\mathrm{~m}, 2 \mathrm{H}), 7.21-7.12(\mathrm{~m}, 3 \mathrm{H}), 6.95-6.90(\mathrm{~m}, 2 \mathrm{H})$, 6.34 (brs, $1 \mathrm{H}) ;{ }^{13} \mathrm{C} \mathrm{NMR}\left(\mathrm{CDCl}_{3}, 75 \mathrm{MHz}\right) \delta 150.3,139.7,139.5,129.8,126.3,124.7,122.0,113.7$; ESIHRMS (m/z) calcd. for $\mathrm{C}_{12} \mathrm{H}_{10} \mathrm{~N}_{2} \mathrm{O}_{2}[(\mathrm{M}+\mathrm{H})]^{+}: 215.08205$, found: 215.08151 .

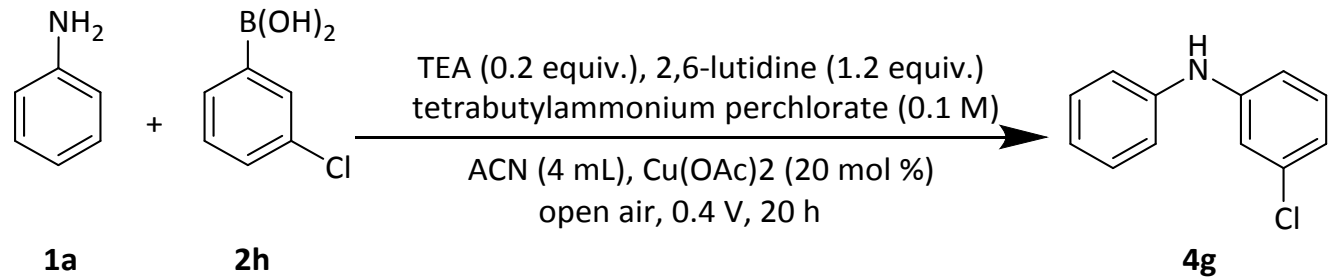

3-Chloro- $\mathbf{N}$-phenylaniline $\mathbf{( 4 g )}$ (Scheme 3). ). Following the above general procedure with 1 a (50 $\mu \mathrm{L}$, $0.55 \mathrm{mmol})$ and $2 \mathrm{~g}(119 \mathrm{mg}, 0.71 \mathrm{mmol})$. The crude reaction mixture was purified by flash chromatography on a $12 \mathrm{~g}$ silica column (0-40\% EtOAc/Heptane) to provide $\mathbf{4 g}$ (84 $\mathrm{mg}, 0.41 \mathrm{mmol}, \mathbf{7 5 \%})$ as a yellow solid. ${ }^{1} \mathrm{H}$ NMR $\left(\mathrm{CDCl}_{3}, 500 \mathrm{MHz}\right) \delta$ 7.28-7.21 $(\mathrm{m}, 2 \mathrm{H})$, 7.11-6.90 $(\mathrm{m}, 5 \mathrm{H}), 6.83-6.77(\mathrm{~m}, 2 \mathrm{H})$, 5.67 (brs, 1H); $\left.{ }^{13} \mathrm{C} \mathrm{NMR} \mathrm{(CDCl}, 125 \mathrm{MHz}\right) \delta$ 144.7, 141.8, 135.0, 129.5, 122.2, 120.5, 119.0, 116.7, 115.2. ESI-HRMS (m/z) calcd. for $\mathrm{C}_{12} \mathrm{H}_{10} \mathrm{CIN}[(\mathrm{M}+\mathrm{H})]^{+}: 204.058$, found: 204.05754 . This is a known compound and the spectral data are identical to those reported in the literature. ${ }^{11}$

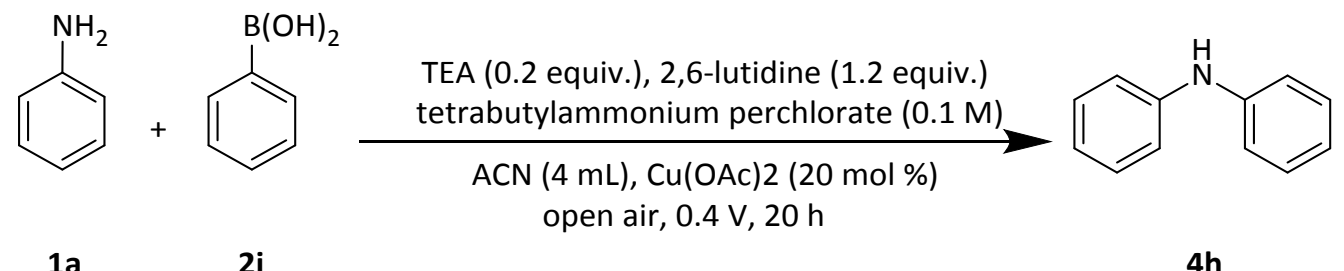

Diphenylamine (4h) (Scheme 3). Following the above general procedure with 1a (50 $\mu \mathrm{L}, 0.55 \mathrm{mmol}$ ) and $2 \mathbf{i}(87 \mathrm{mg}, 0.71 \mathrm{mmol})$. ). The crude reaction mixture was purified by flash chromatography on a $12 \mathrm{~g}$ 
silica column (0-40\% EtOAc/Heptane) to provide $\mathbf{4 h}(81 \mathrm{mg}, 0.48 \mathrm{mmol}, \mathbf{8 7 \%})$ as a yellow solid. ${ }^{1} \mathrm{H}$ NMR $\left(\mathrm{CDCl}_{3}, 300 \mathrm{MHz}\right) \delta 7.28(\mathrm{t}, \mathrm{J}=7.9 \mathrm{~Hz}, 4 \mathrm{H}), 7.08(\mathrm{~d}, \mathrm{~J}=7.9 \mathrm{~Hz}, 4 \mathrm{H}), 6.94(\mathrm{t}, \mathrm{J}=7.4 \mathrm{~Hz}, 2 \mathrm{H}), 5.70(\mathrm{brs}, 1 \mathrm{H})$; ${ }^{13} \mathrm{C} \mathrm{NMR}\left(\mathrm{CDCl}_{3}, 75 \mathrm{MHz}\right) \delta 143.0,129.3,120.9$, 117.7. ESI-HRMS (m/z) calcd. for $\mathrm{C}_{12} \mathrm{H}_{11} \mathrm{~N}[(\mathrm{M}+\mathrm{H})]^{+}$: 170.09697, found: 170.09639. This is a known compound and the spectral data are identical to those reported in the literature. ${ }^{3}$

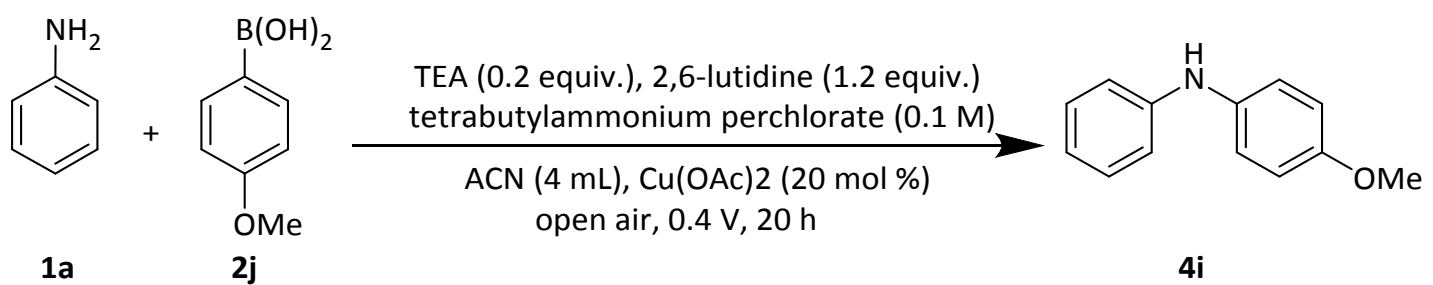

4-Methoxy- $\mathbf{N}$-phenylaniline (4i) (Scheme 3). Following the above general procedure with 1 a (50 $\mu \mathrm{L}$, $0.55 \mathrm{mmol}$ ) and $2 \mathrm{j}(108 \mathrm{mg}, 0.71 \mathrm{mmol})$. The crude reaction mixture was purified by flash chromatography on a $12 \mathrm{~g}$ silica column (0-40\% EtOAc/Heptane) to provide $4 \mathbf{i}(77 \mathrm{mg}, 0.39 \mathrm{mmol}, \mathbf{7 0 \%})$ as a yellow solid. ${ }^{1} \mathrm{H}$ NMR $\left(\mathrm{CDCl}_{3}, 300 \mathrm{MHz}\right) \delta=7.28-7.21(\mathrm{~m}, 2 \mathrm{H}), 7.12-7.08(\mathrm{~m}, 2 \mathrm{H}), 6.95-6.85(\mathrm{~m}, 5 \mathrm{H})$, 5.52 (brs, 1H), 3.82 (s, 3H); $\left.{ }^{13} \mathrm{C} \mathrm{NMR} \mathrm{(CDCl}, 75 \mathrm{MHz}\right) \delta 155.2,145.1,135.6,129.3,122.2,119.5,115.6$, 114.6, 55.5. ESI-HRMS (m/z) calcd. for $\mathrm{C}_{13} \mathrm{H}_{13} \mathrm{NO}[(\mathrm{M}+\mathrm{H})]^{+}: 200.10699$, found: 200.10652 . This is a known compound and the spectral data are identical to those reported in the literature. ${ }^{4}$

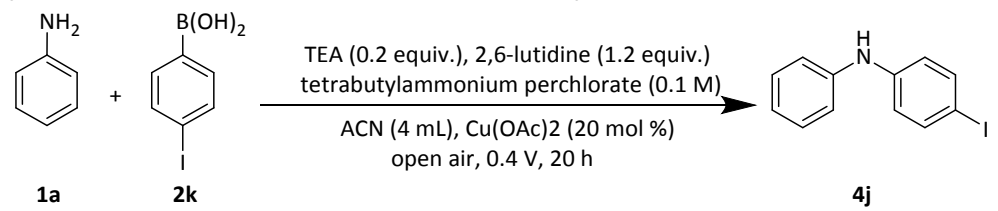

4-iodo-N-phenyl-aniline (4j) (Scheme 3) Following the above general procedure with 1a (50 $\mu \mathrm{L}, 0.55$ $\mathrm{mmol}$ ) and $2 \mathbf{k}(177 \mathrm{mg}, 0.71 \mathrm{mmol})$. The crude reaction mixture was purified by flash chromatography on a $12 \mathrm{~g}$ silica column (0-40\% EtOAc/Heptane) to provide $4 \mathrm{j}$ (117 $\mathrm{mg}, 0.40 \mathrm{mmol}, \mathbf{7 2} \%)$ as a brown solid. ${ }^{1} \mathrm{H} \mathrm{NMR}\left(\mathrm{CDCl}_{3}, 300 \mathrm{MHz}\right) \delta$ 7.48-7.44 $(\mathrm{m}, 2 \mathrm{H}), 7.23-7.20(\mathrm{~m}, 2 \mathrm{H}), 7.03-6.99(\mathrm{~m}, 2 \mathrm{H}), 6.96-6.89(\mathrm{~m}, 1 \mathrm{H})$,

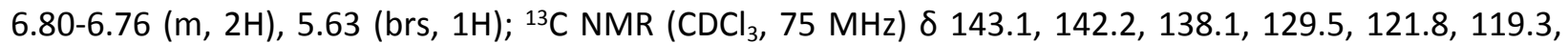
118.5, 81.1. ESI-HRMS (m/z) calcd. for $\mathrm{C}_{12} \mathrm{H}_{10} \mathrm{IN}[(\mathrm{M}+\mathrm{H})]^{+}: 295.99307$, found: 295.99349.

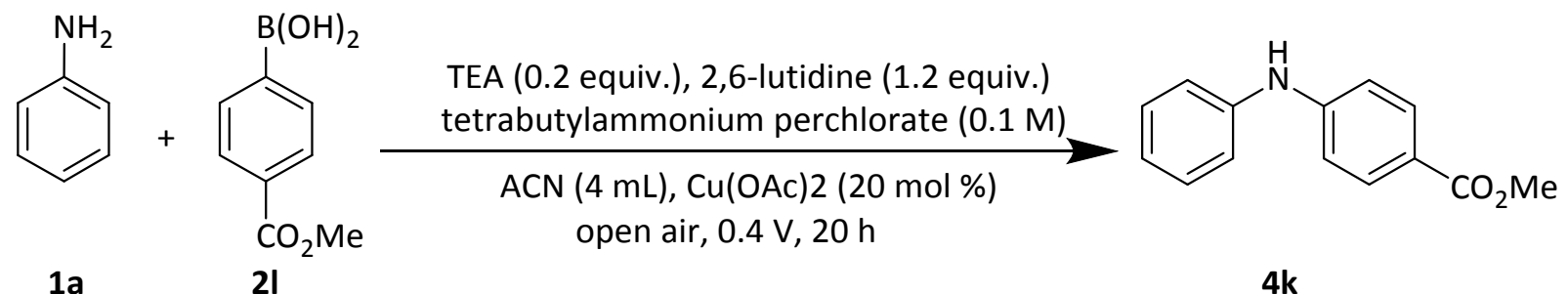

4-Methoxy-N-phenylaniline (4k) (Scheme 3). Following the above general procedure with 1a (50 $\mu \mathrm{L}$, $0.55 \mathrm{mmol})$ and $2 \mathrm{l}(129 \mathrm{mg}, 0.71 \mathrm{mmol})$. The crude reaction mixture was purified by flash chromatography on a $12 \mathrm{~g}$ silica column (0-40\% EtOAc/Heptane) to provide $4 \mathbf{k}$ (74 $\mathrm{mg}, 0.33 \mathrm{mmol}, 60 \%$ ) as a white solid. ${ }^{1} \mathrm{H}$ NMR $(300 \mathrm{MHz}$, Chloroform-d) $\delta 7.96-7.85(\mathrm{~m}, 2 \mathrm{H}), 7.41-7.28(\mathrm{~m}, 2 \mathrm{H}), 7.21-7.12$ $(\mathrm{m}, 2 \mathrm{H}), 7.12-7.03(\mathrm{~m}, 1 \mathrm{H}), 7.02-6.95(\mathrm{~m}, 2 \mathrm{H}), 6.07$ (brs, $1 \mathrm{H}), 3.88(\mathrm{~s}, 3 \mathrm{H}) ;{ }^{13} \mathrm{C} \mathrm{NMR}(75 \mathrm{MHz}$, Chloroform-d) $\delta 167.0,148.1,140.9,131.5,129.5,123.1,121.1,120.5,114.6,51.8$. ESI-HRMS (m/z) calcd. for $\mathrm{C}_{13} \mathrm{H}_{13} \mathrm{NO}[(\mathrm{M}+\mathrm{H})]^{+}: 200.10699$, found: 200.10652 . This is a known compound and the spectral data are identical to those reported in the literature. ${ }^{4}$ 


\section{Procedure for Photochemical Chan-Lam: ${ }^{12}$}

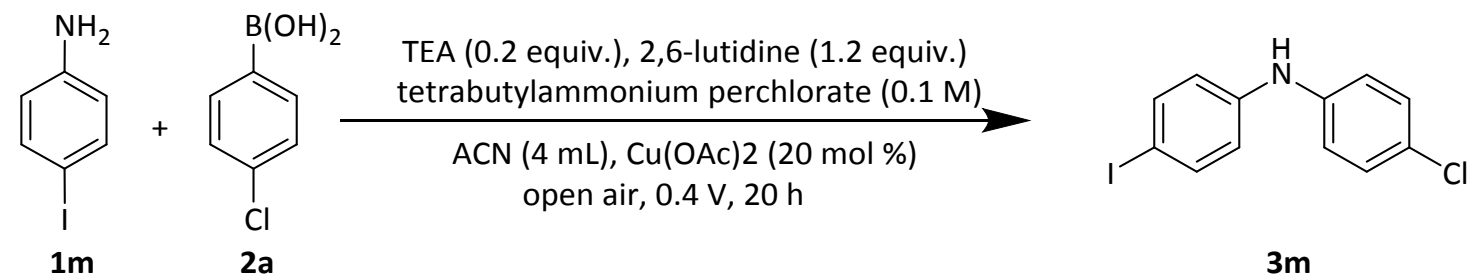

Diacetoxycopper (20 mg, $0.11 \mathrm{mmol}$ ), iridium(3+);1-phenylisoquinoline (1 mg, $0.001 \mathrm{mmol}$ ), $1 \mathrm{~m} \mathrm{(147} \mathrm{mg,}$ $0.67 \mathrm{mmol}$ ) were added to a $1 \mathrm{dram}$ vial. Added 2,6-lutidine $(80 \mu \mathrm{L}, 0.69 \mathrm{mmol})$, tetradecanoic acid (25 $\mathrm{mg}, 0.11 \mathrm{mmol})$ and $2 \mathrm{a}(112 \mathrm{mg}, 0.72 \mathrm{mmol})$. Dissolved in toluene $(1 \mathrm{~mL})$ and acetonitrile $(1 \mathrm{~mL})$ then capped with a septum. Punctured with a needle to open to oxygen and irradiated via Kessel lamp with blue LEDs for 24 hours. Filtered over celite after diluting with ethyl acetate then purified the crude residue by silca gel chromatography (0-40\% EA/Heptane) to yield 4-chloro- $\mathrm{N}$-(4-iodophenyl)aniline $3 \mathrm{~m}$ (50 mg, $0.11 \mathrm{mmol}, \mathbf{2 1 \%}$ ). Spectra identical to reported above for $\mathbf{3 m}$.

Procedure for the synthesis of 4-Chloro- $\mathrm{N}$-phenylaniline on $\mathrm{mmol}$ scale:<smiles>Nc1ccccc1</smiles>

$1 a$<smiles>Oc1ccc(Br)cc1</smiles>

2a
TEA ( 0.2 equiv.), 2,6-lutidine (1.2 equiv.)

Tetrabutylammonium Perchlorate (0.1 M)

ACN (4 mL), Cu(OAc)2 (20 mol \%)

Open to air, $0.4 \mathrm{~V}, 20 \mathrm{~h}$<smiles>Clc1ccc(Nc2ccccc2)cc1</smiles>

3a

In a $20 \mathrm{~mL}$ IKA-Works ElectraSyn vial was added $\mathrm{Cu}(\mathrm{OAc})_{2}(40 \mathrm{mg}, 0.22 \mathrm{mmol}, 20 \mathrm{~mol} \%$ ), tetrabutylammonium perchlorate $(275 \mathrm{mg}, 0.80 \mathrm{mmol}, 0.1 \mathrm{M})$, triethylamine $(30 \mu \mathrm{L}, 0.22 \mathrm{mmol}, 0.2$ equiv.), 2,6-lutidine (155 $\mu \mathrm{L}, 0.132 \mathrm{mmol}), A C N(8 \mathrm{~mL})$, arylboronic acid $2 \mathrm{a}(222 \mathrm{mg}, 0.142 \mathrm{mmol})$, and aniline derivative 1a $(100 \mu \mathrm{L}, 1.1 \mathrm{mmol})$. The vial was left open to atmosphere and was stirred for 20 hours under a potential of $0.40 \mathrm{~V}$. The crude reaction mixture was then diluted with ethyl acetate and passed through a $7 \mathrm{~g}$ silica plug to remove copper. Next, the reaction mixture was blown down, taken up in minimal DCM, and purified by flash chromatography on a $24 \mathrm{~g}$ silica column (0-40\% EtOAc/Heptane). After removal of solvent, 4-Chloro- $N$-phenylaniline $\mathbf{3 a}(185 \mathrm{mg}, 0.913 \mathrm{mmol}, \mathbf{8 3} \%)$ eluted as a yellow oil. 


\section{Copies of NMR Spectra:}

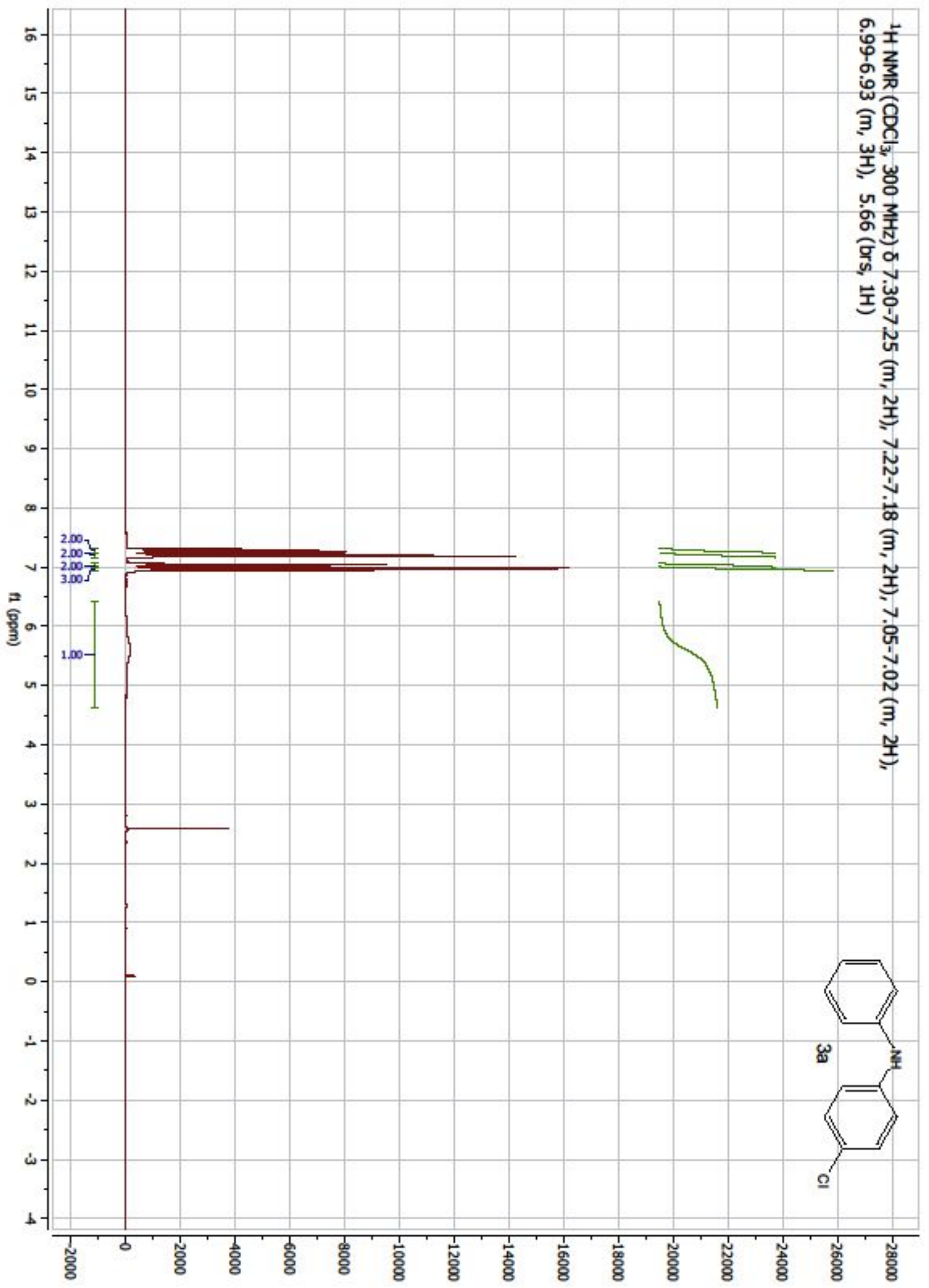




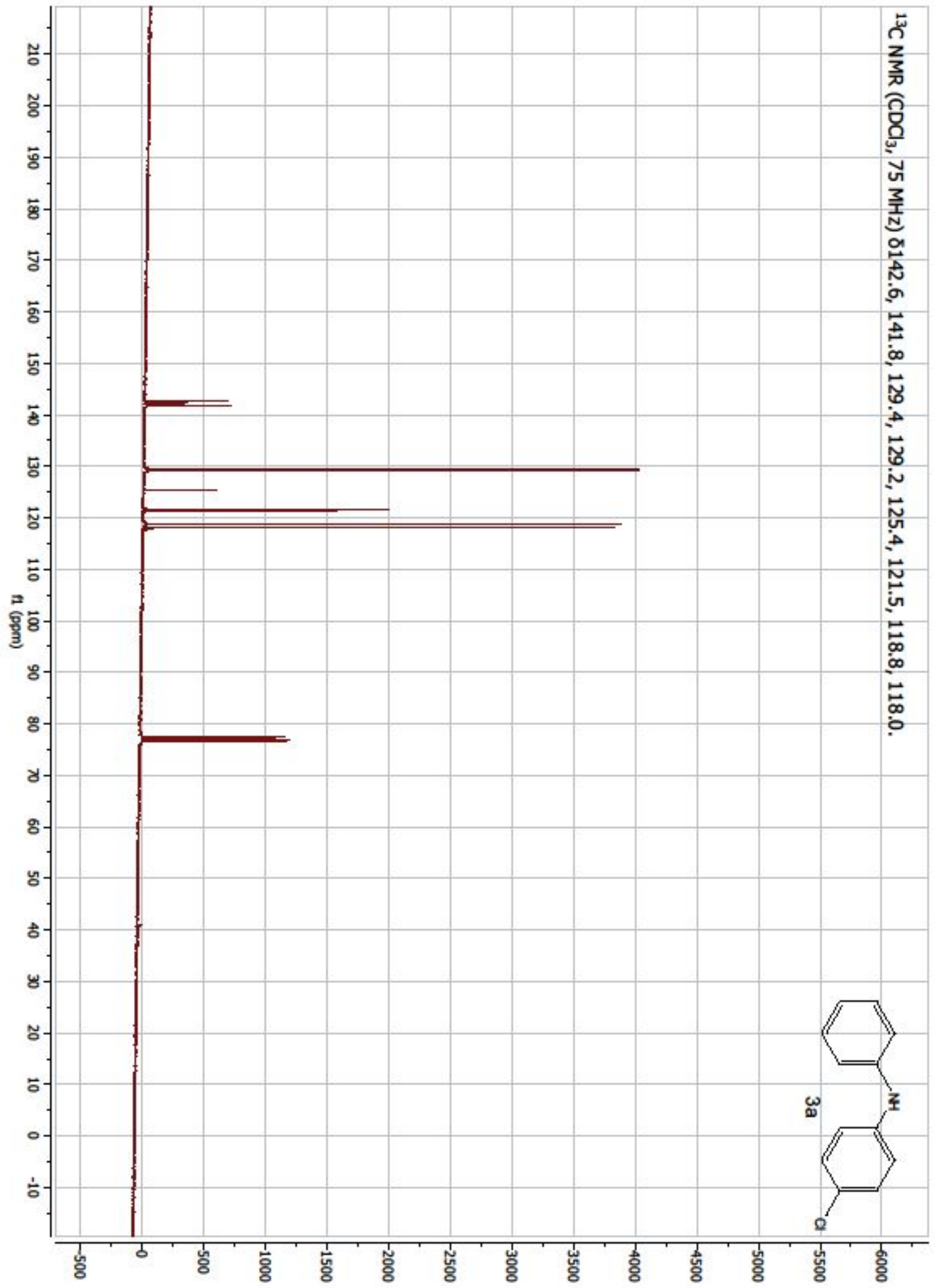




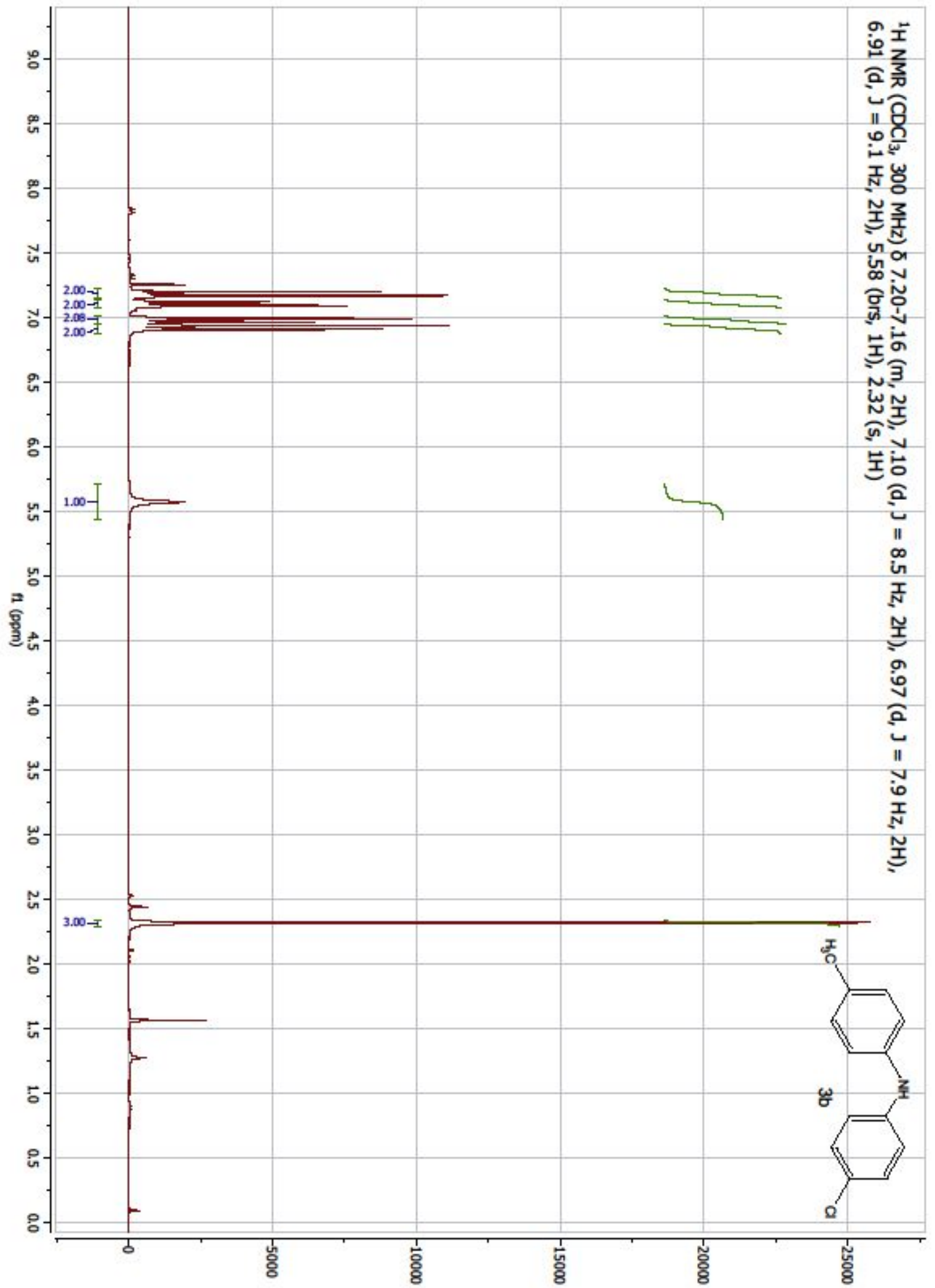




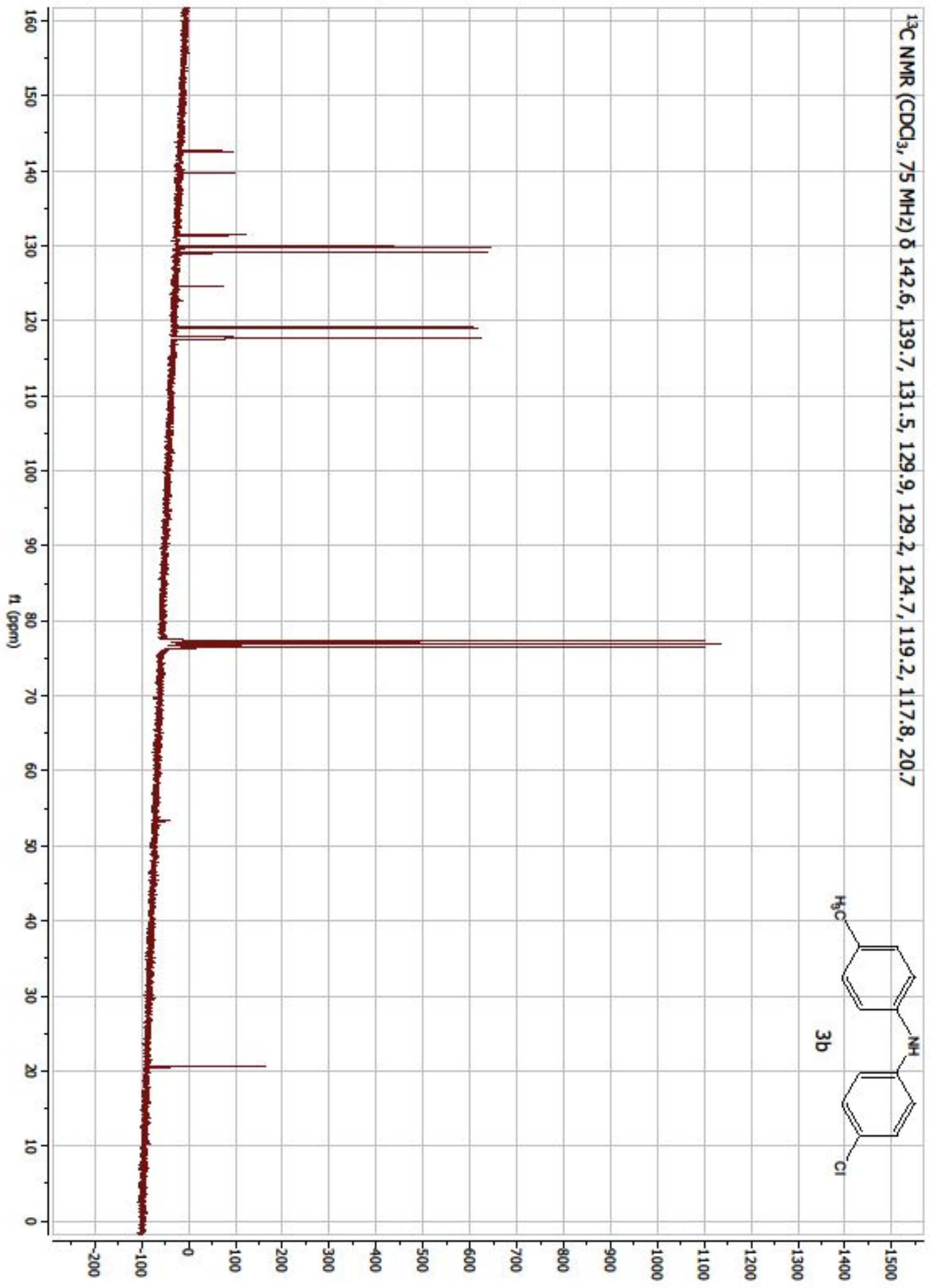




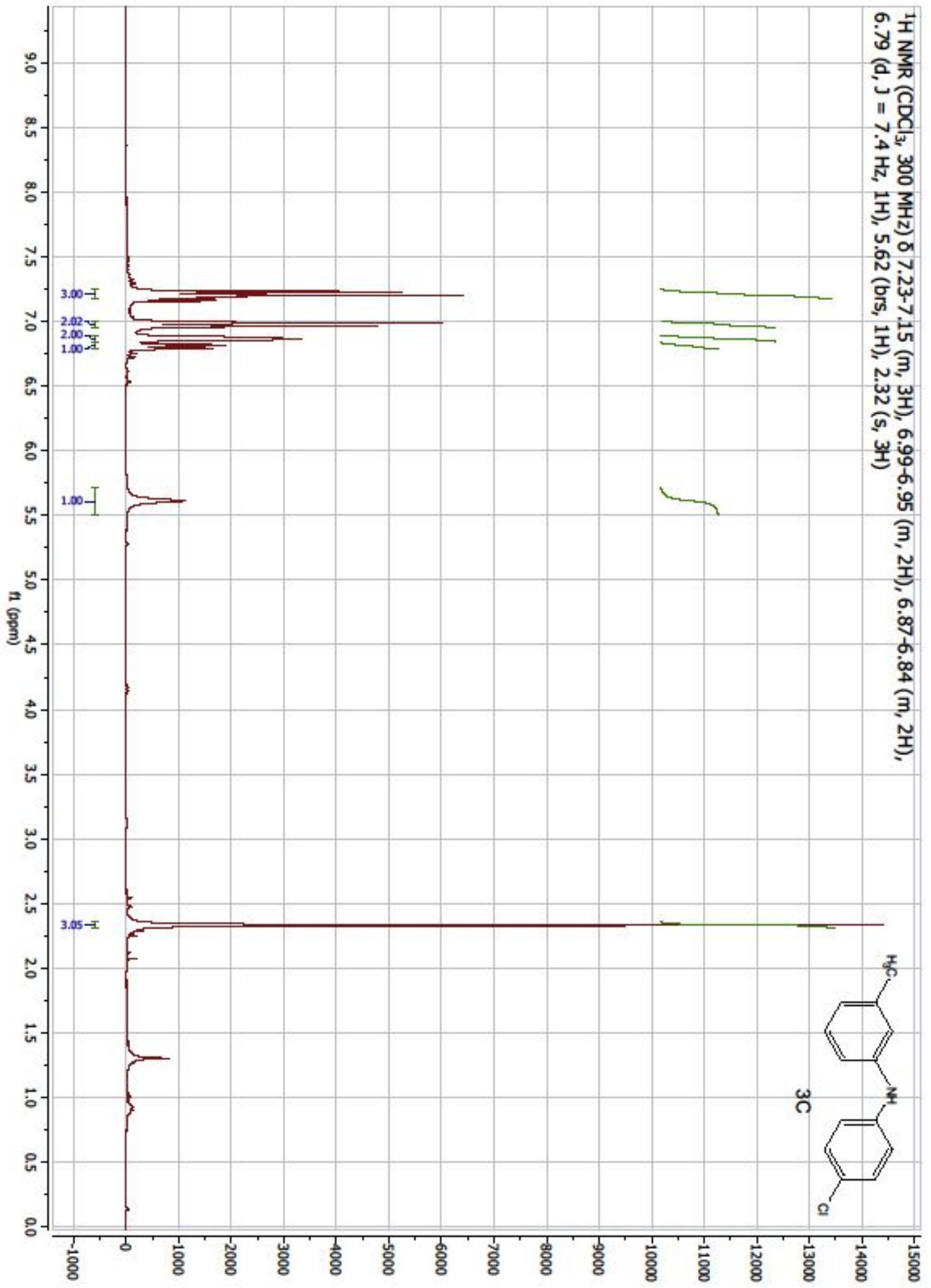




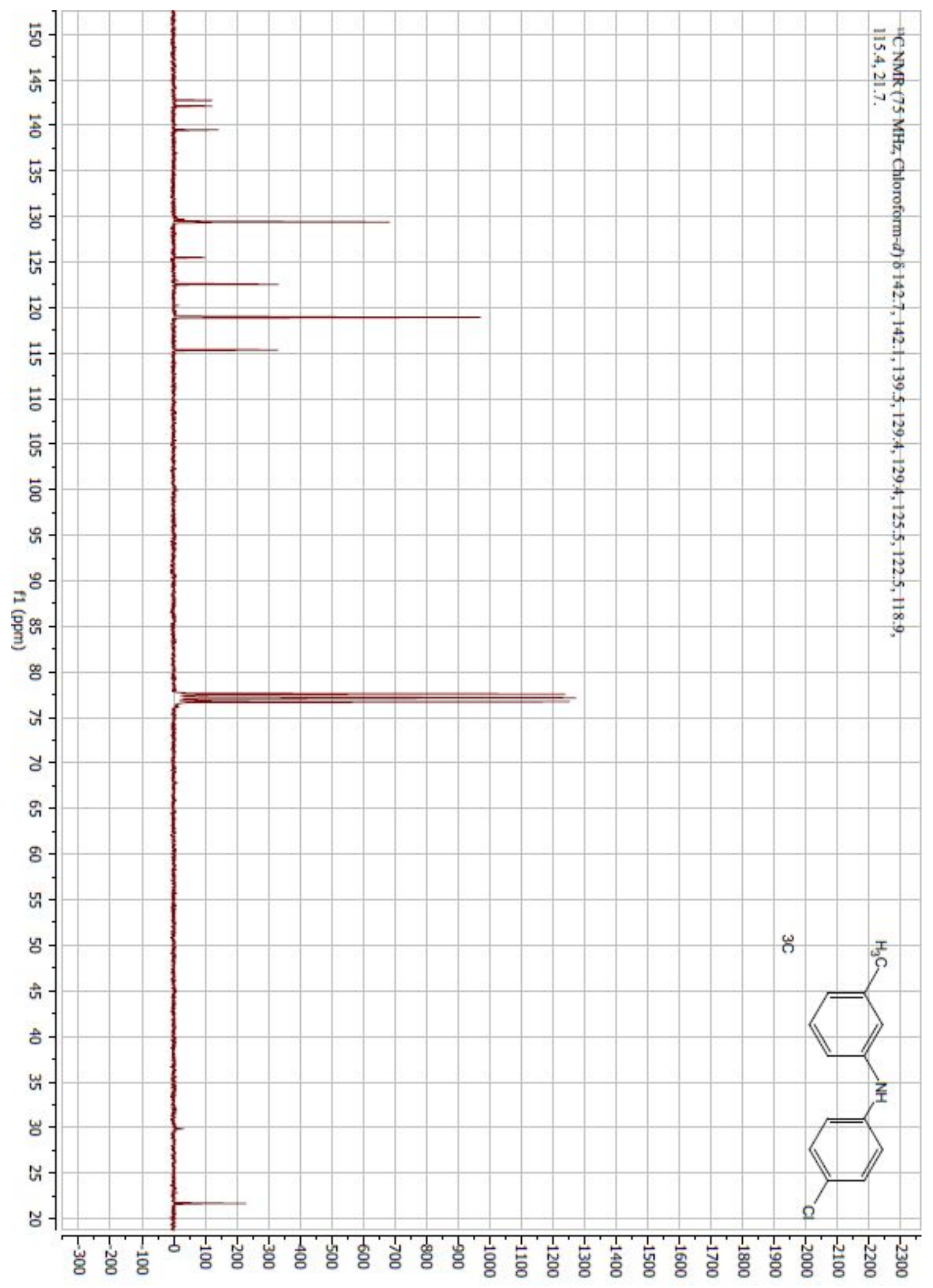




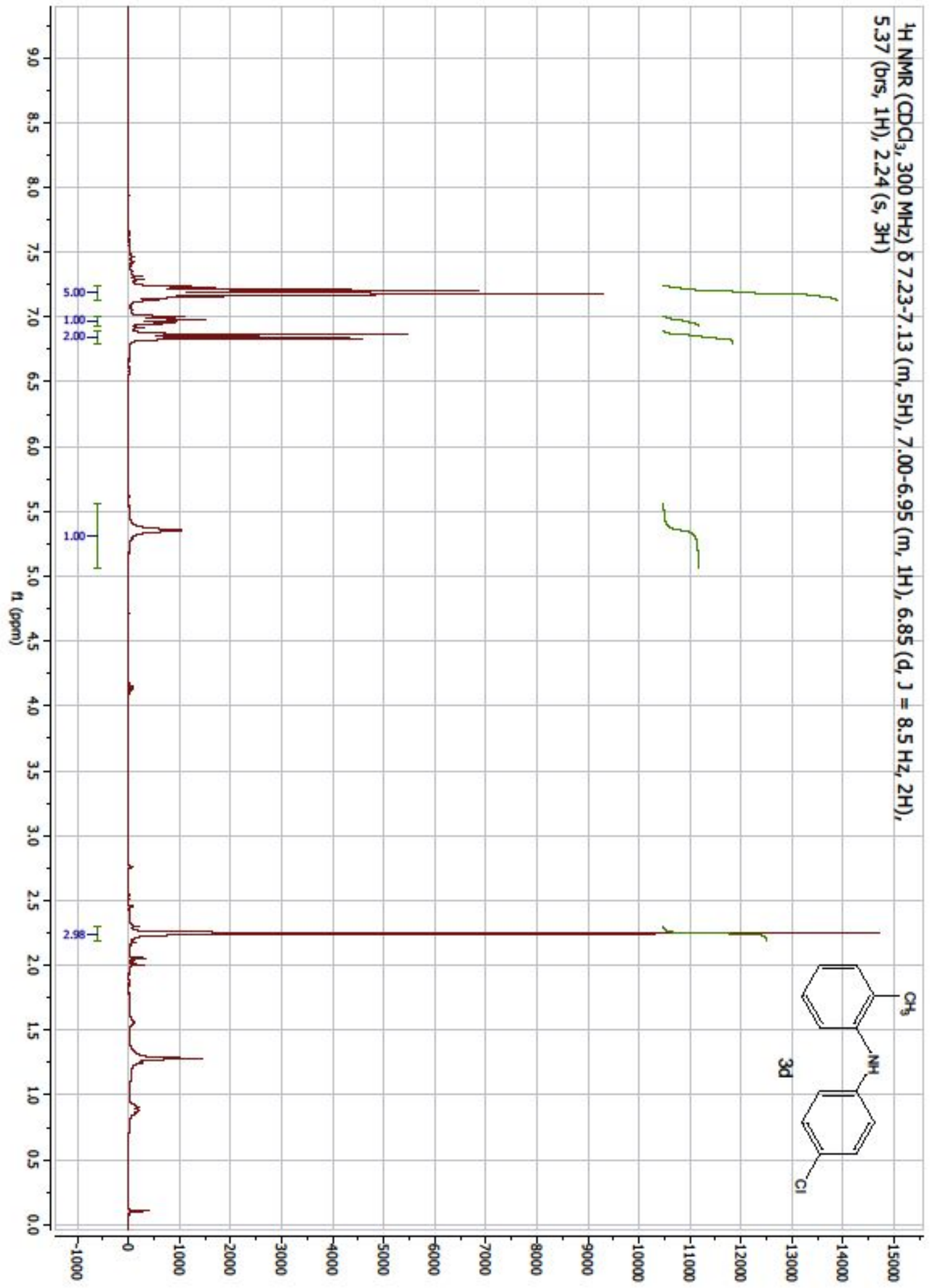




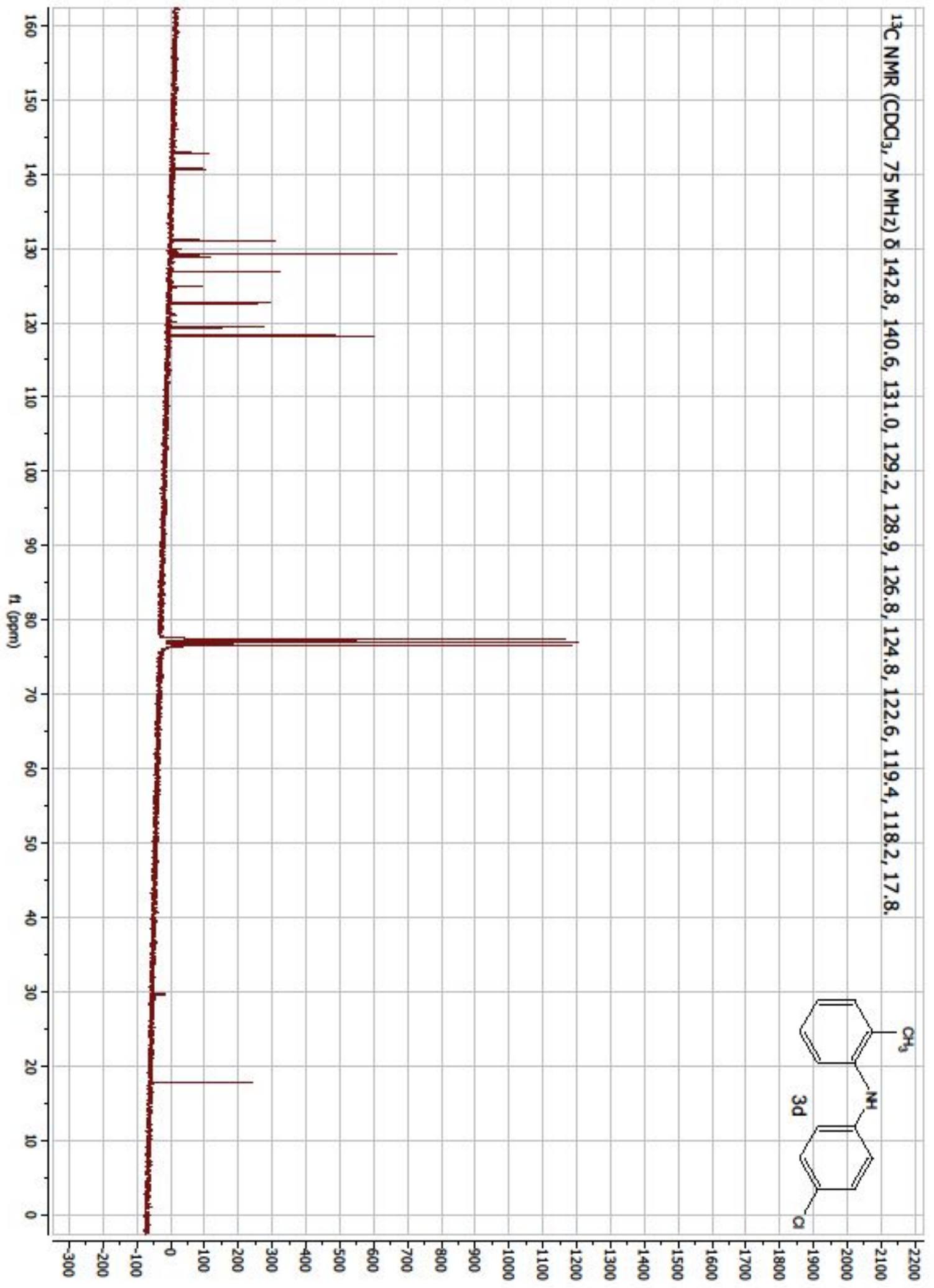




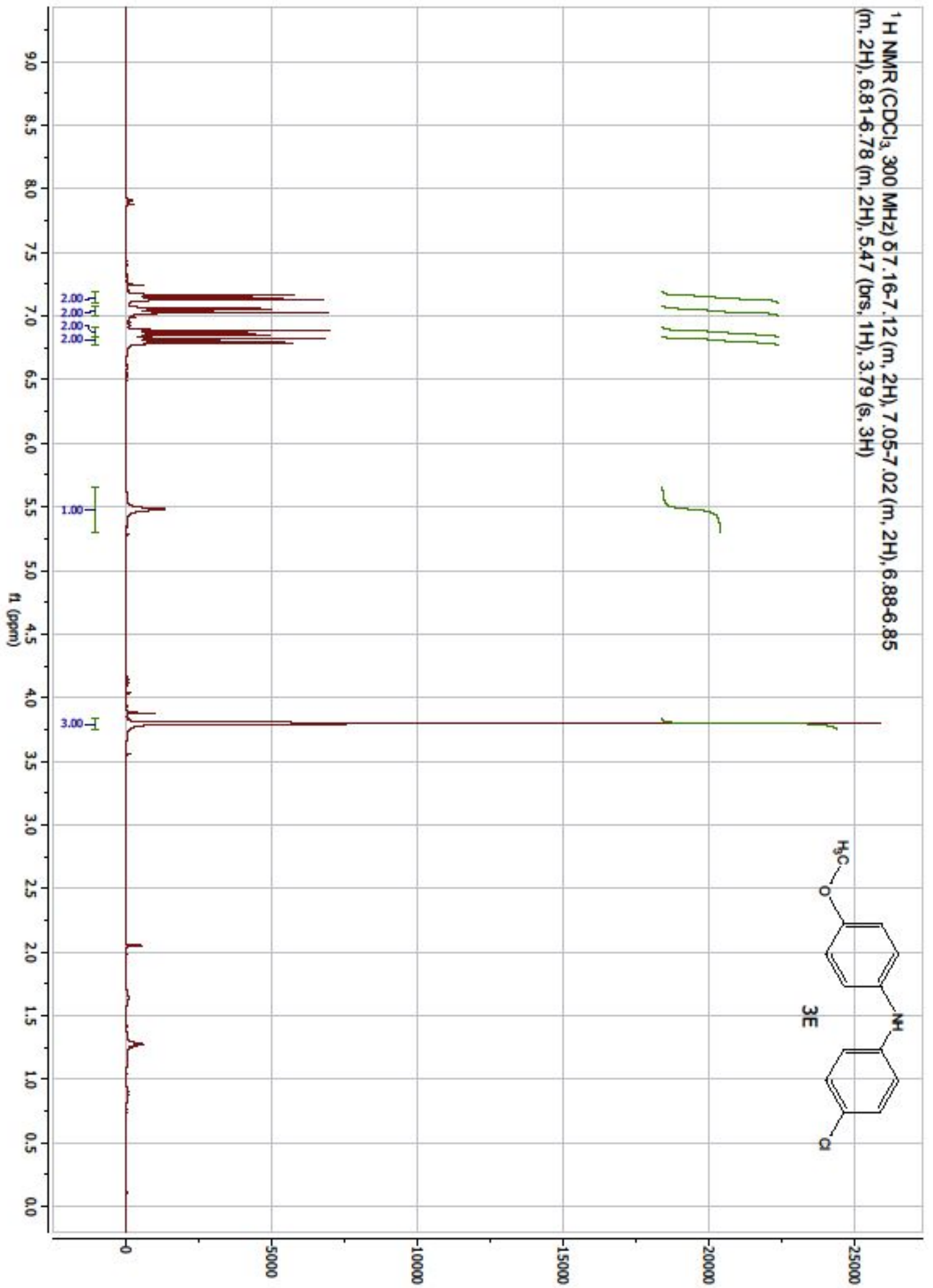




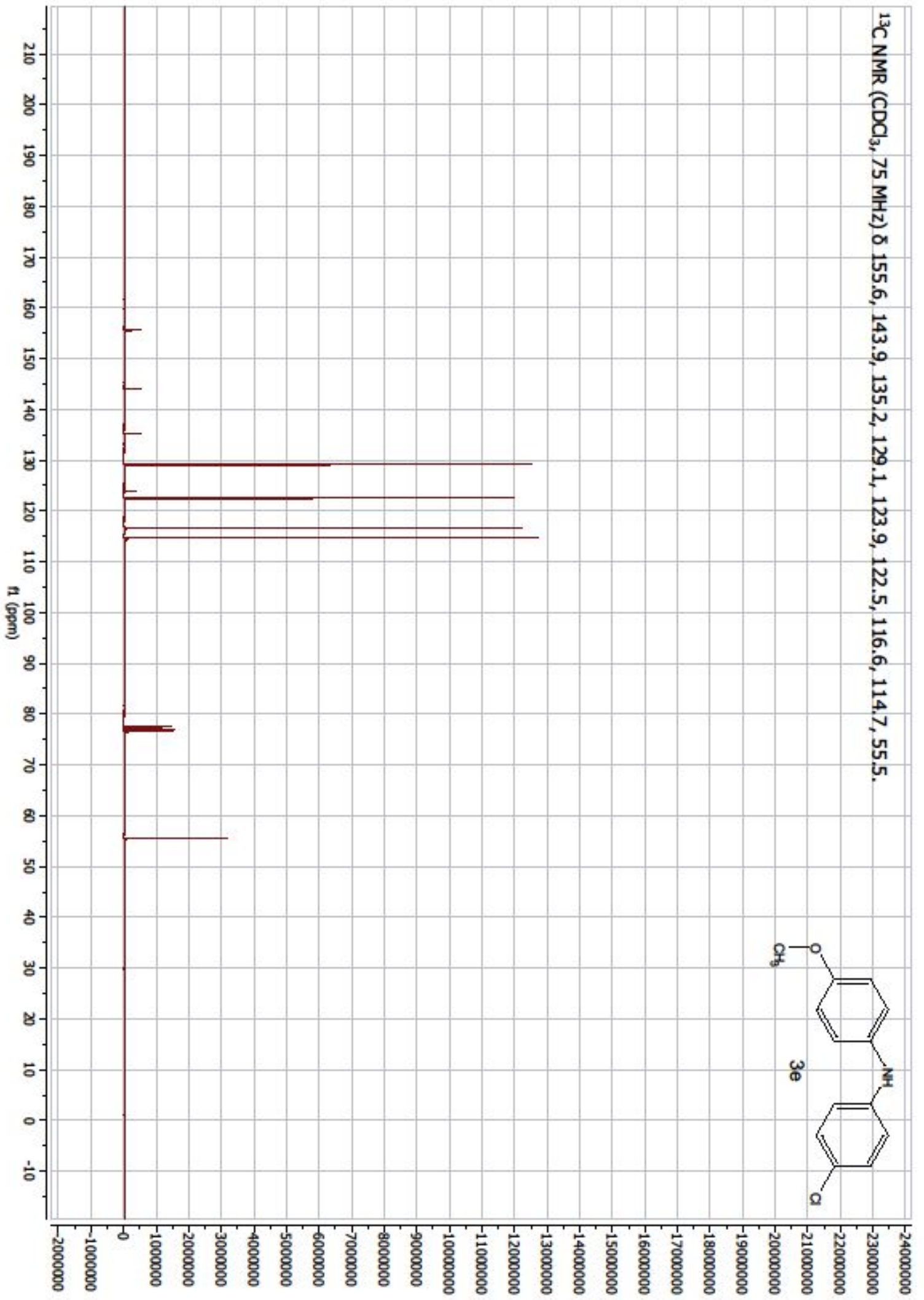




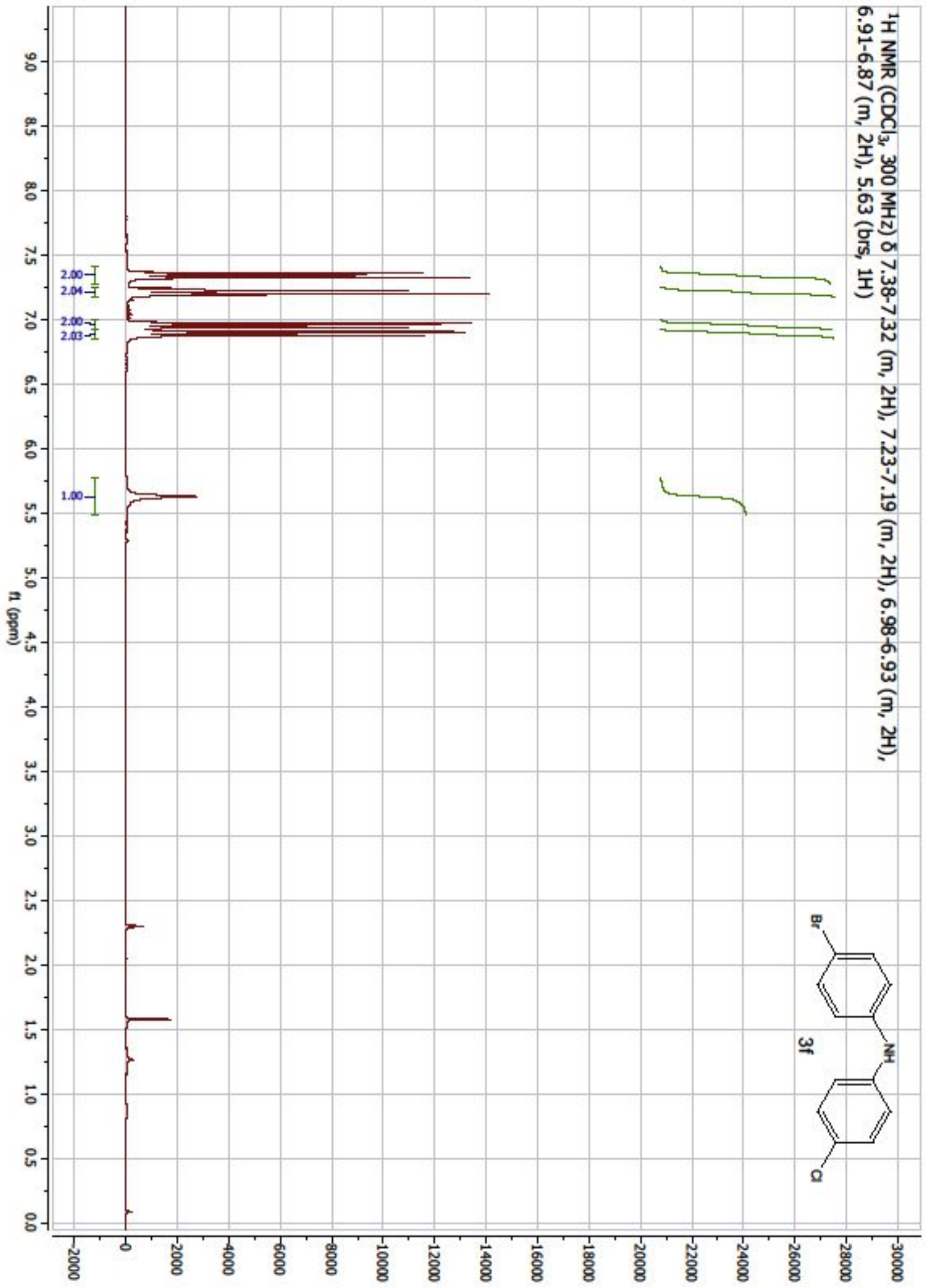




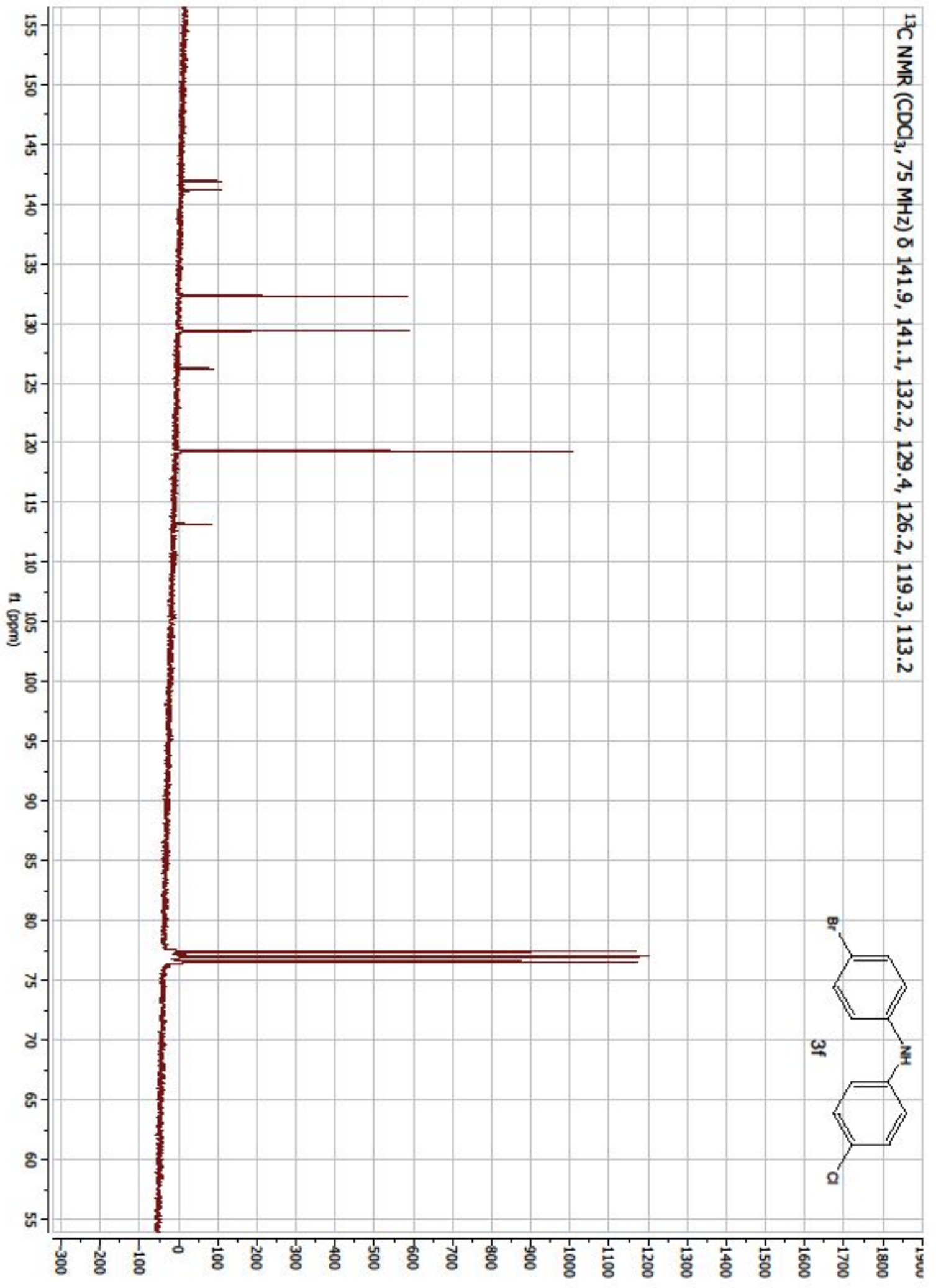




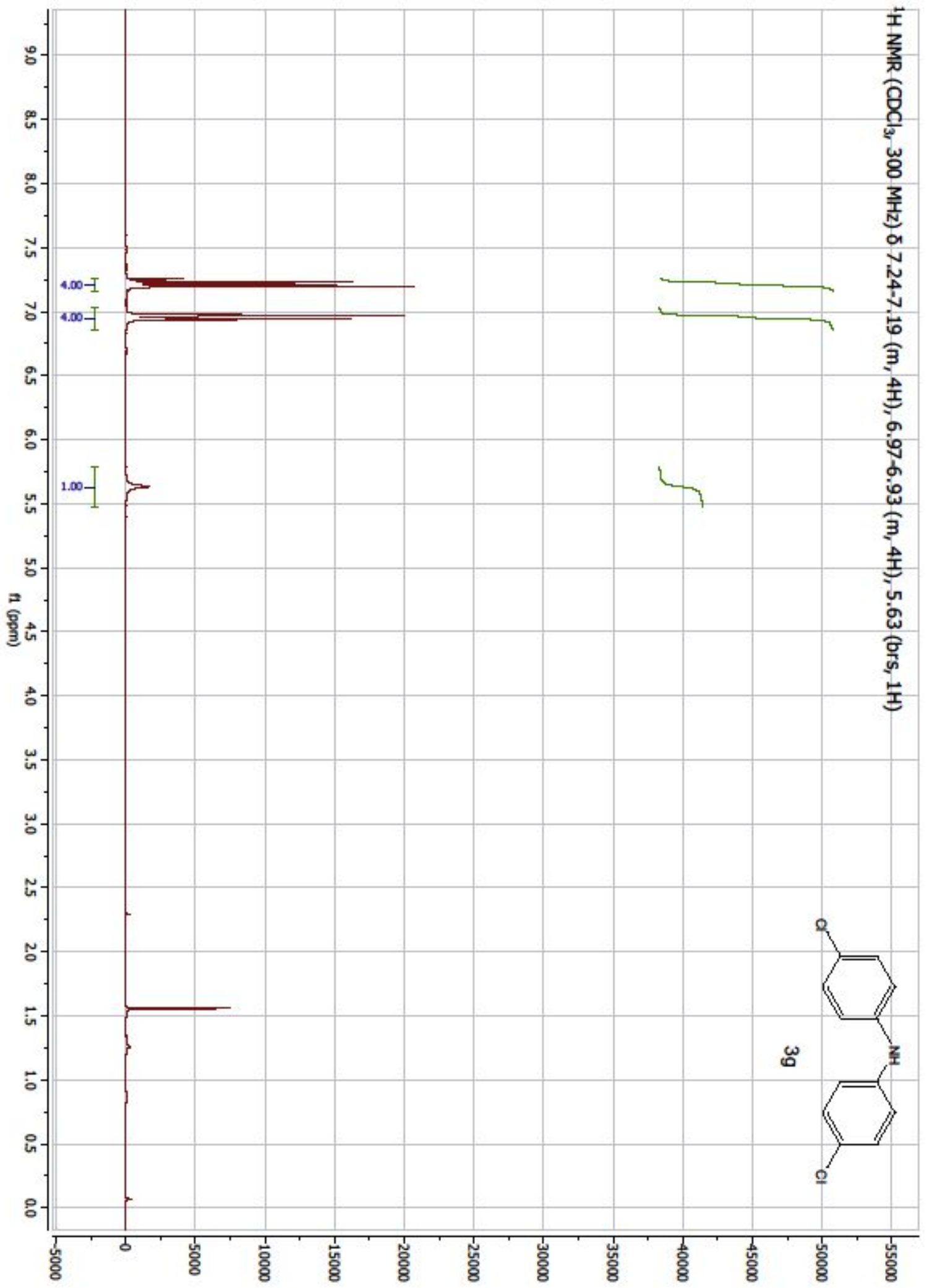




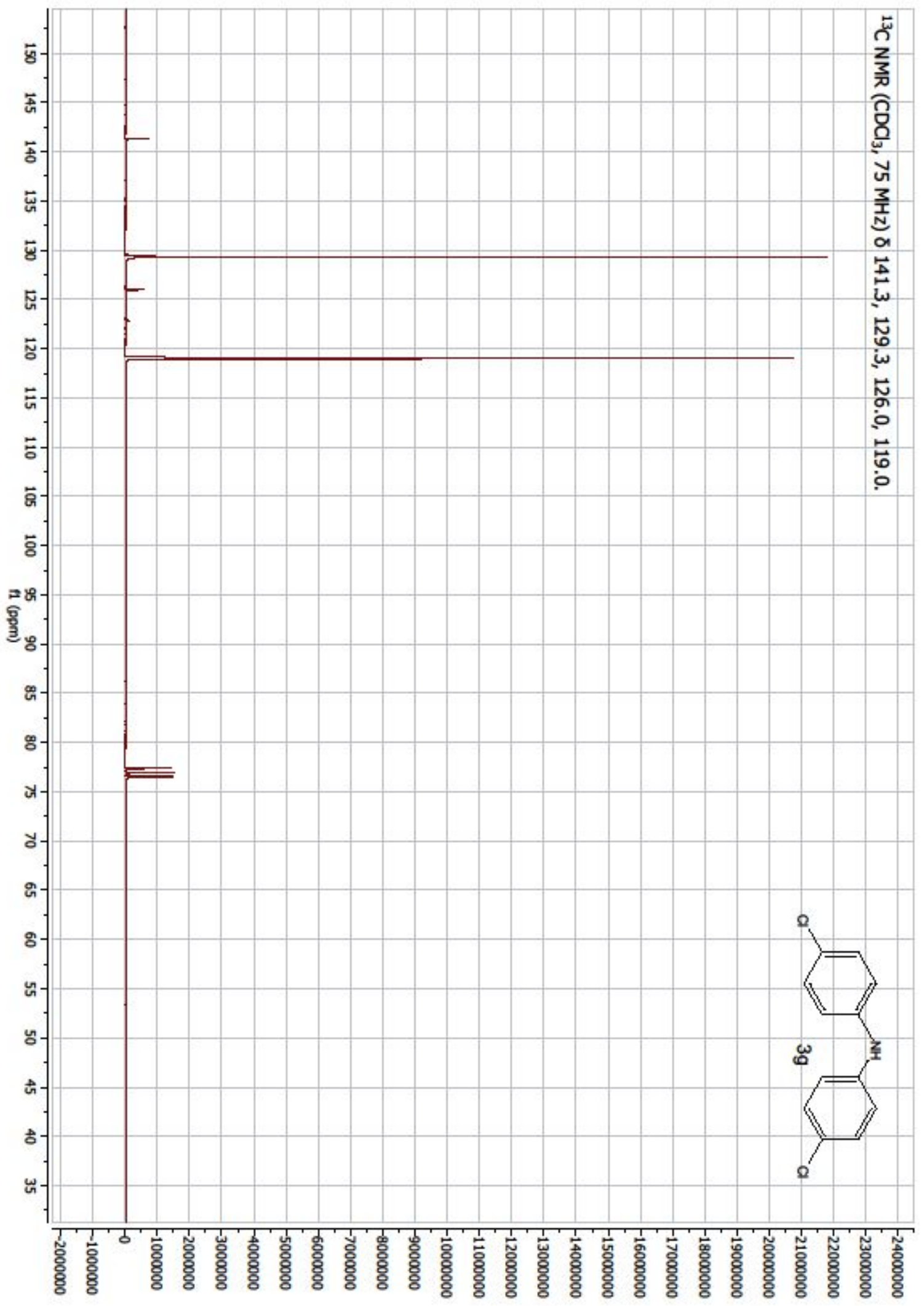




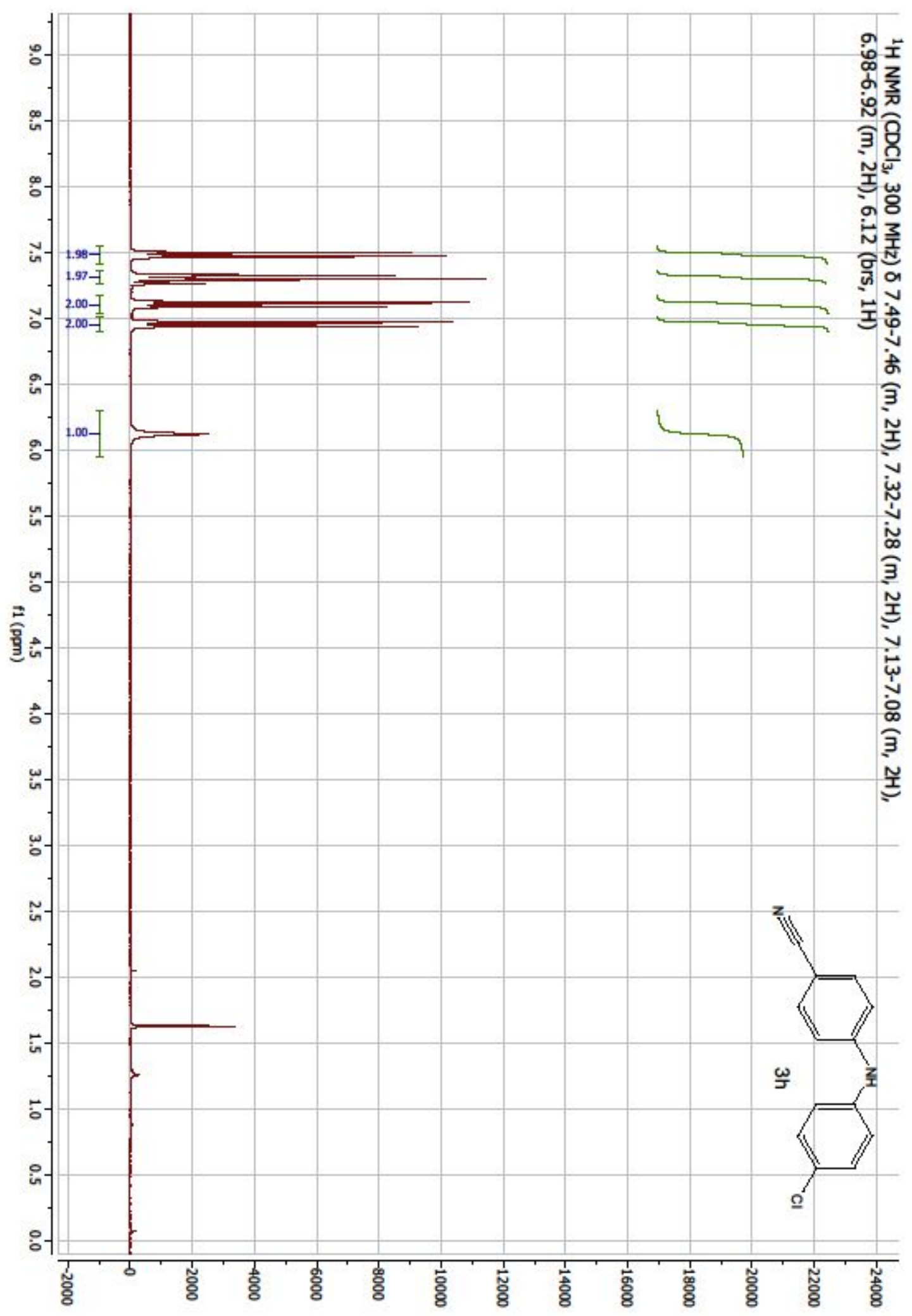




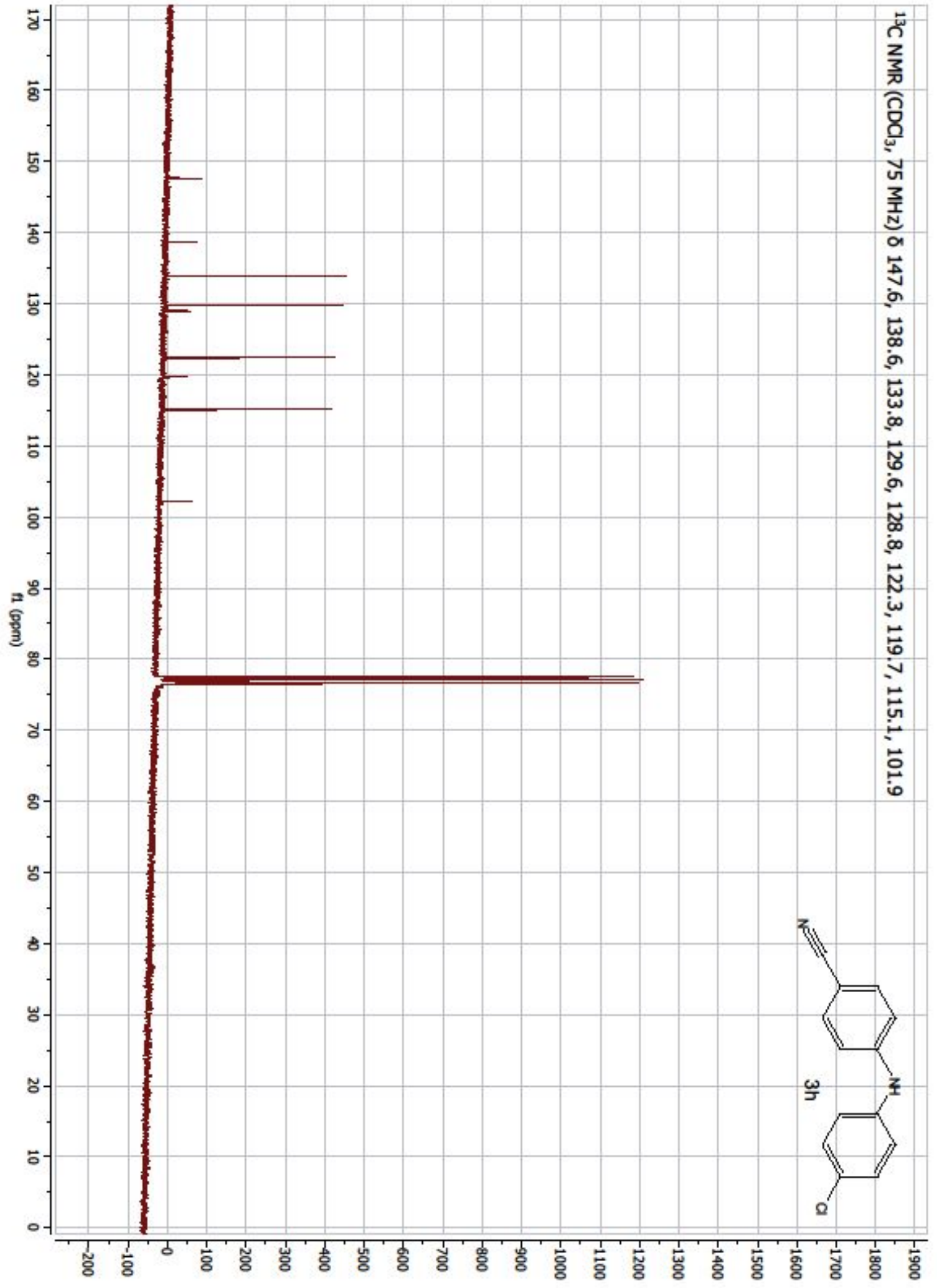




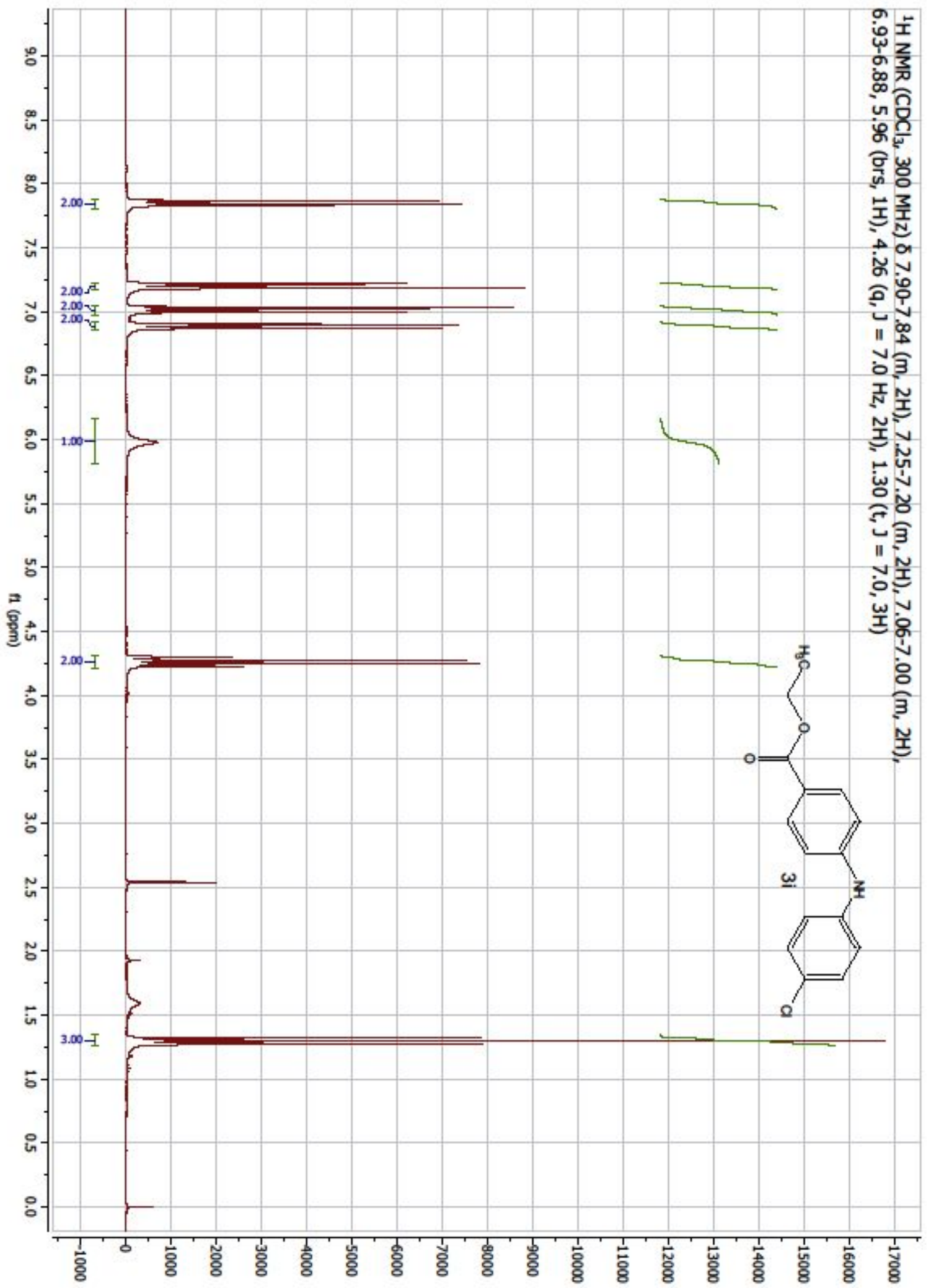




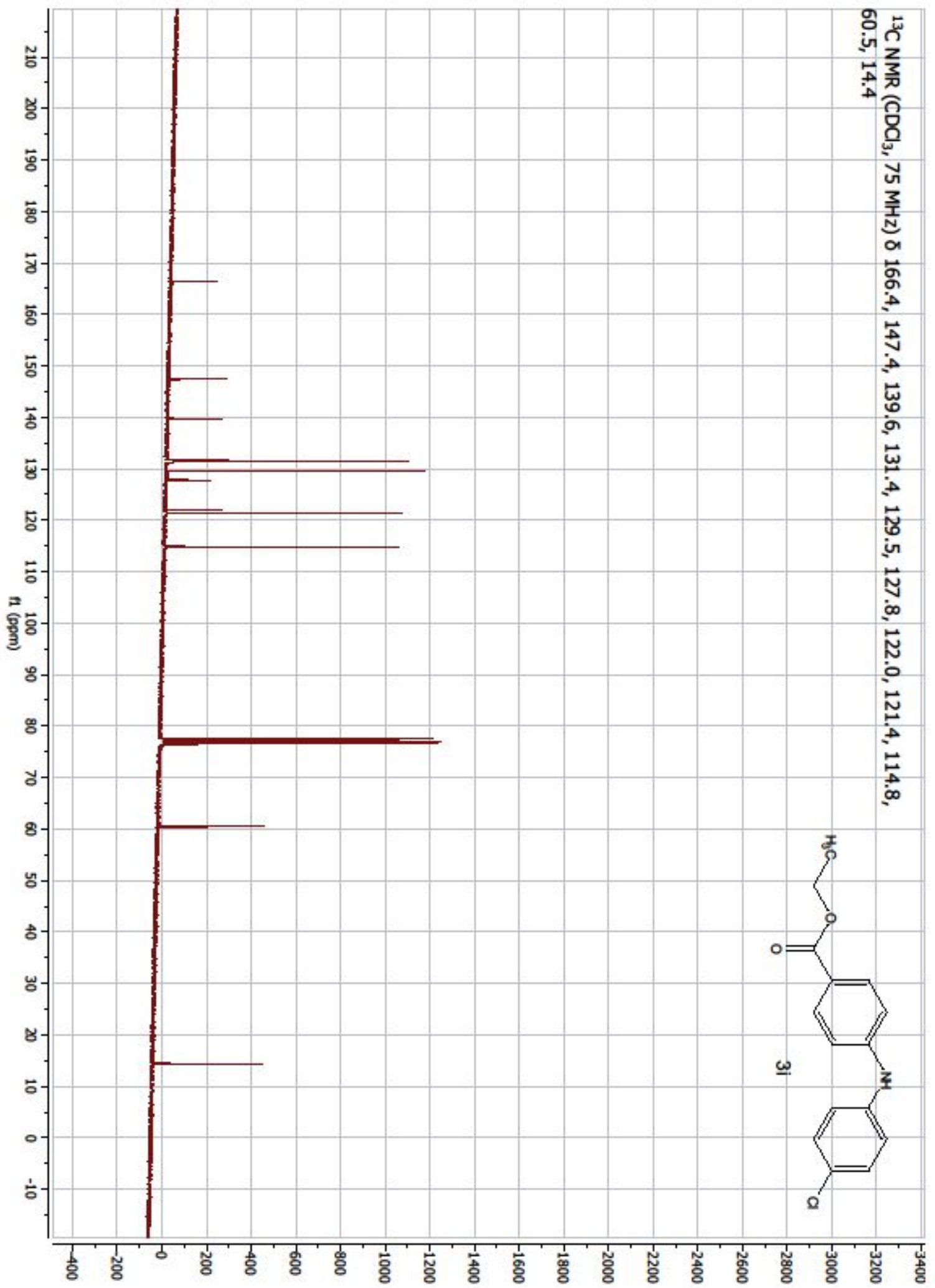




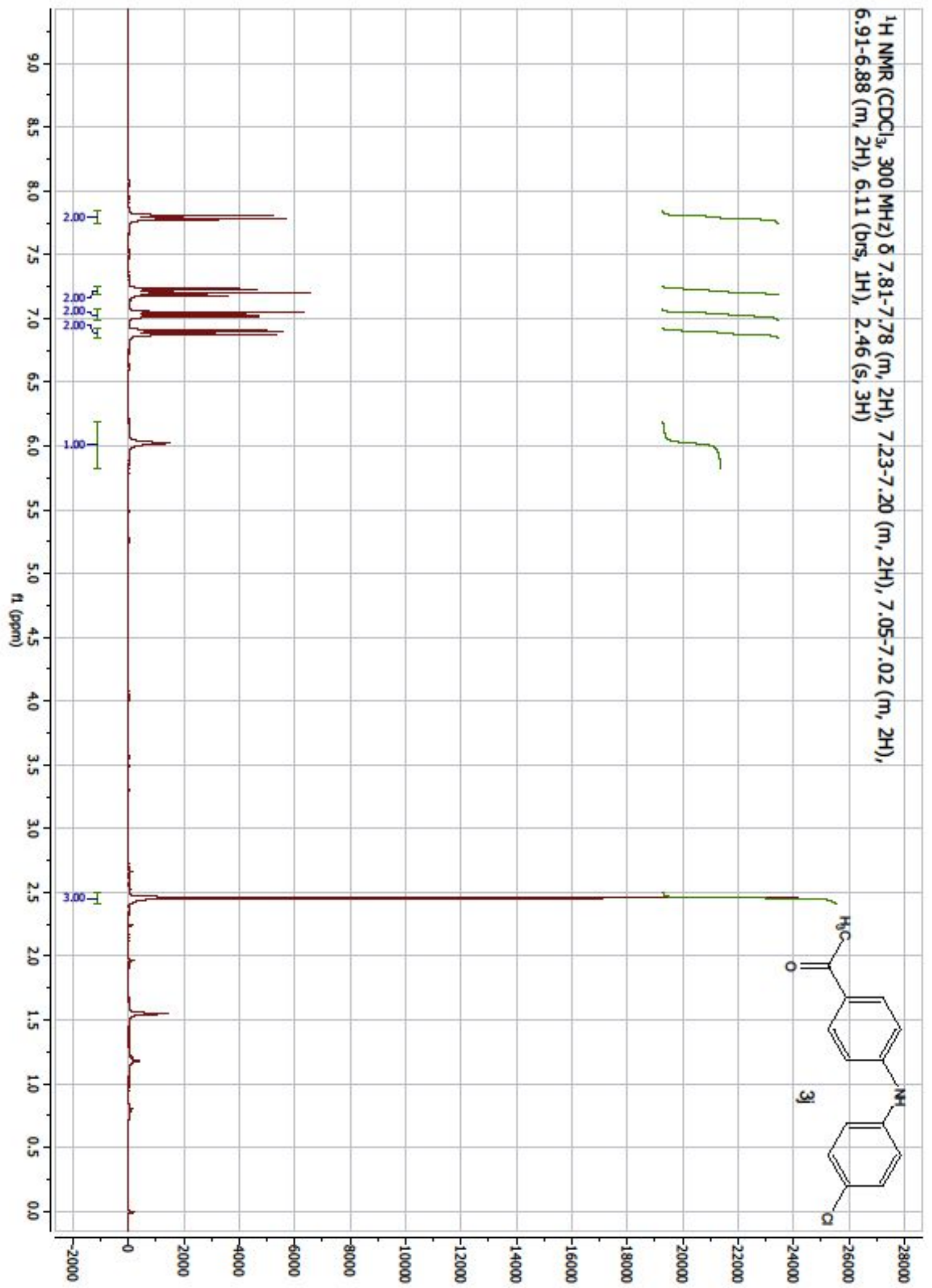




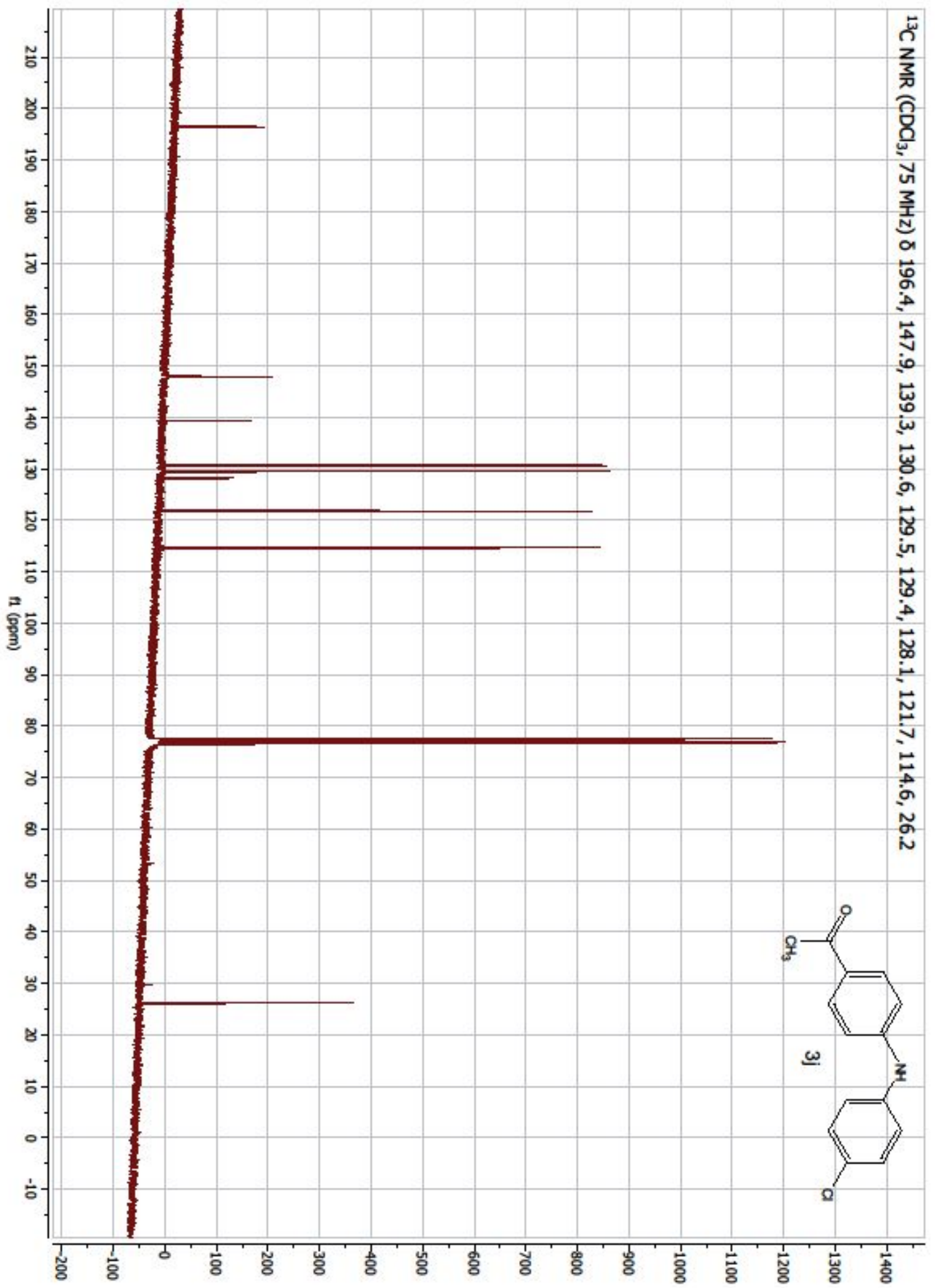




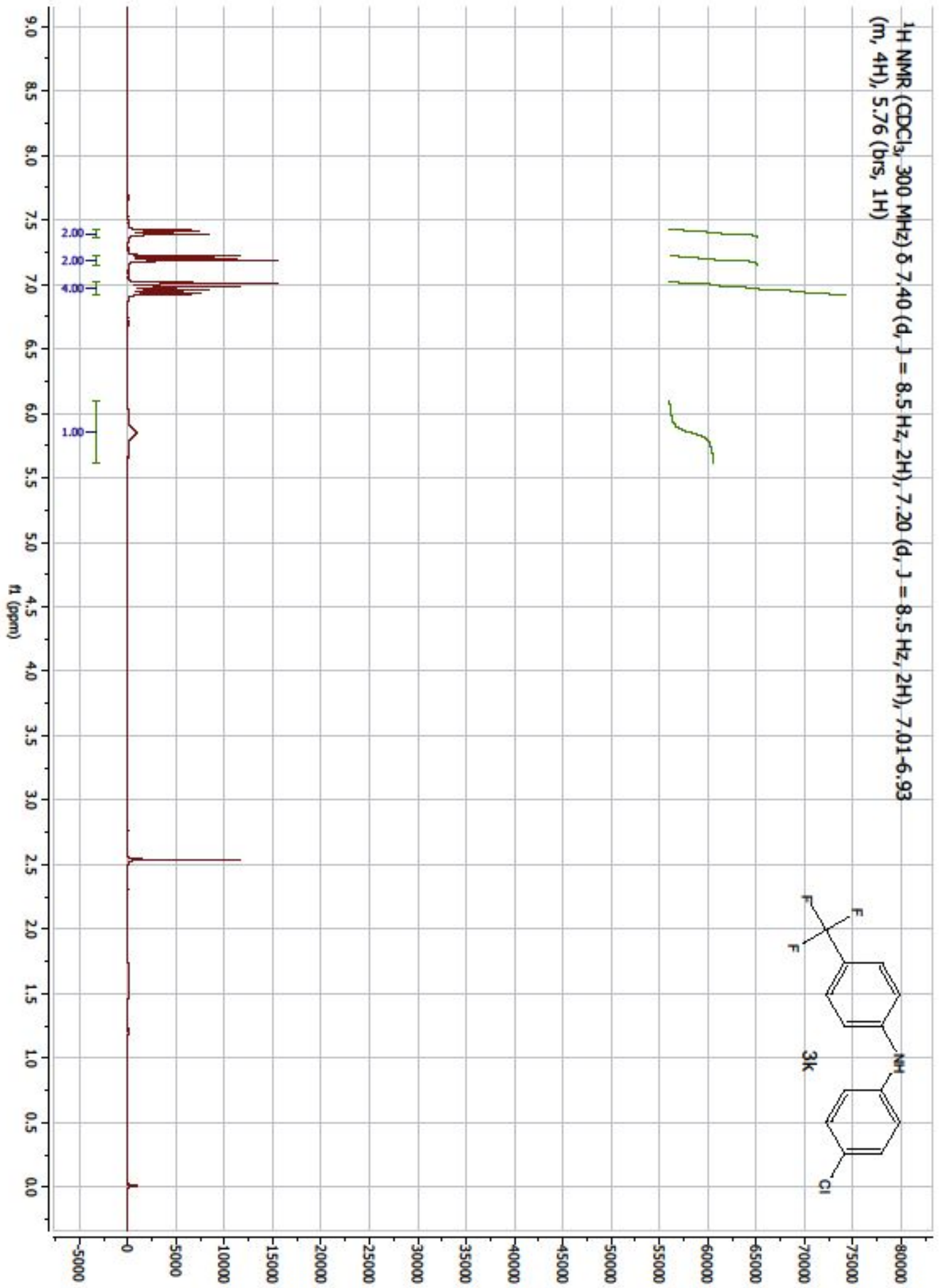




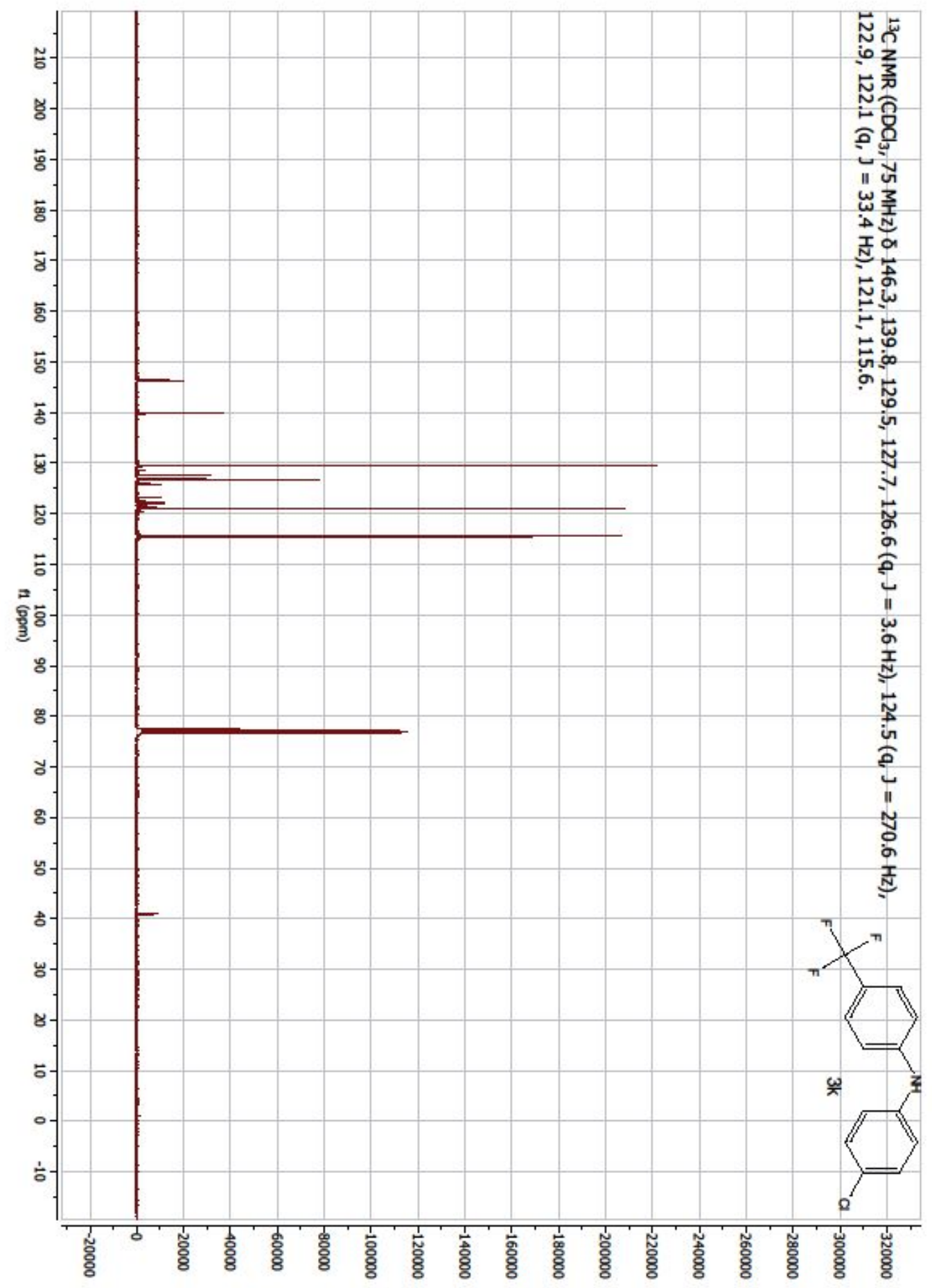




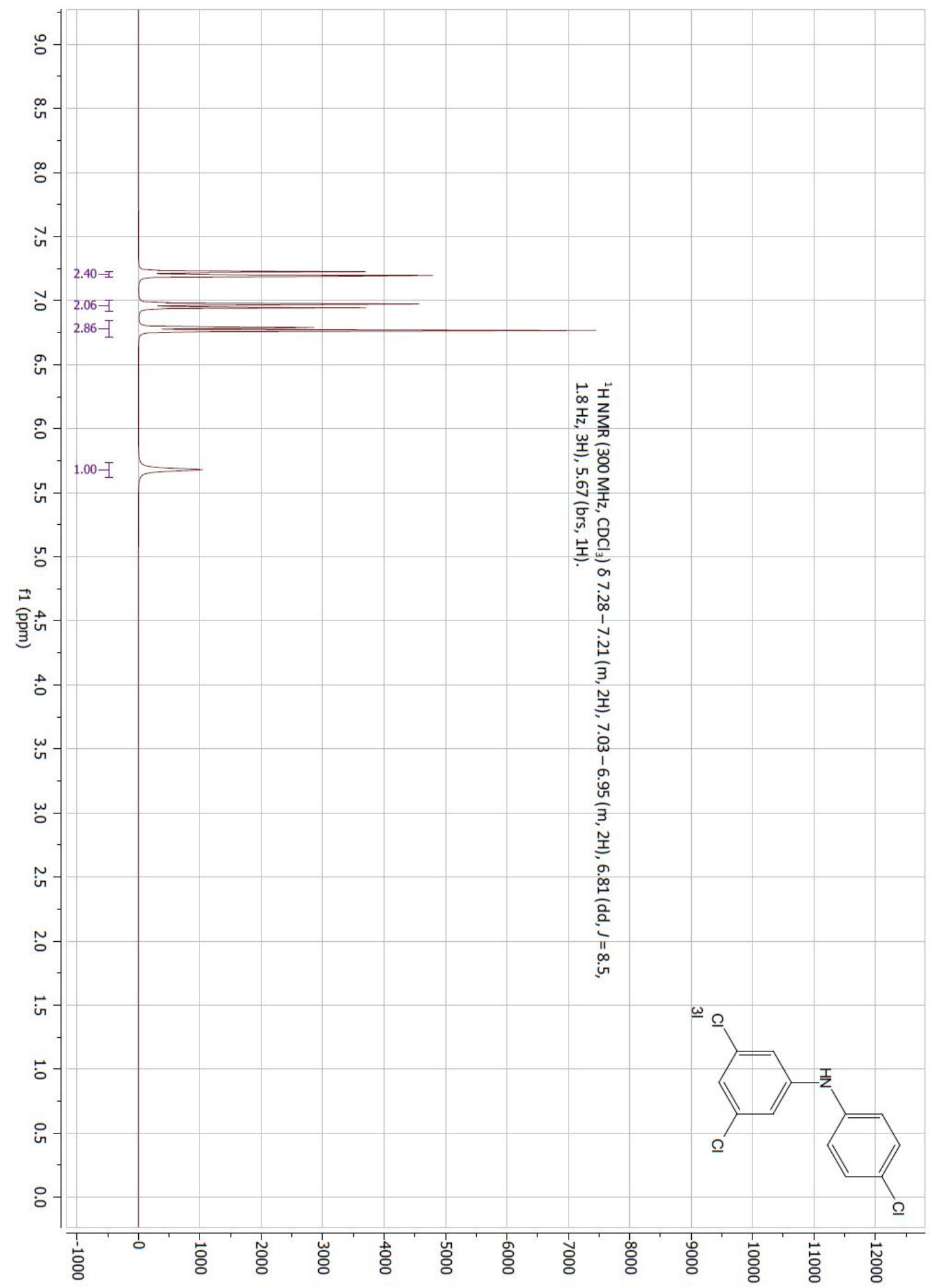




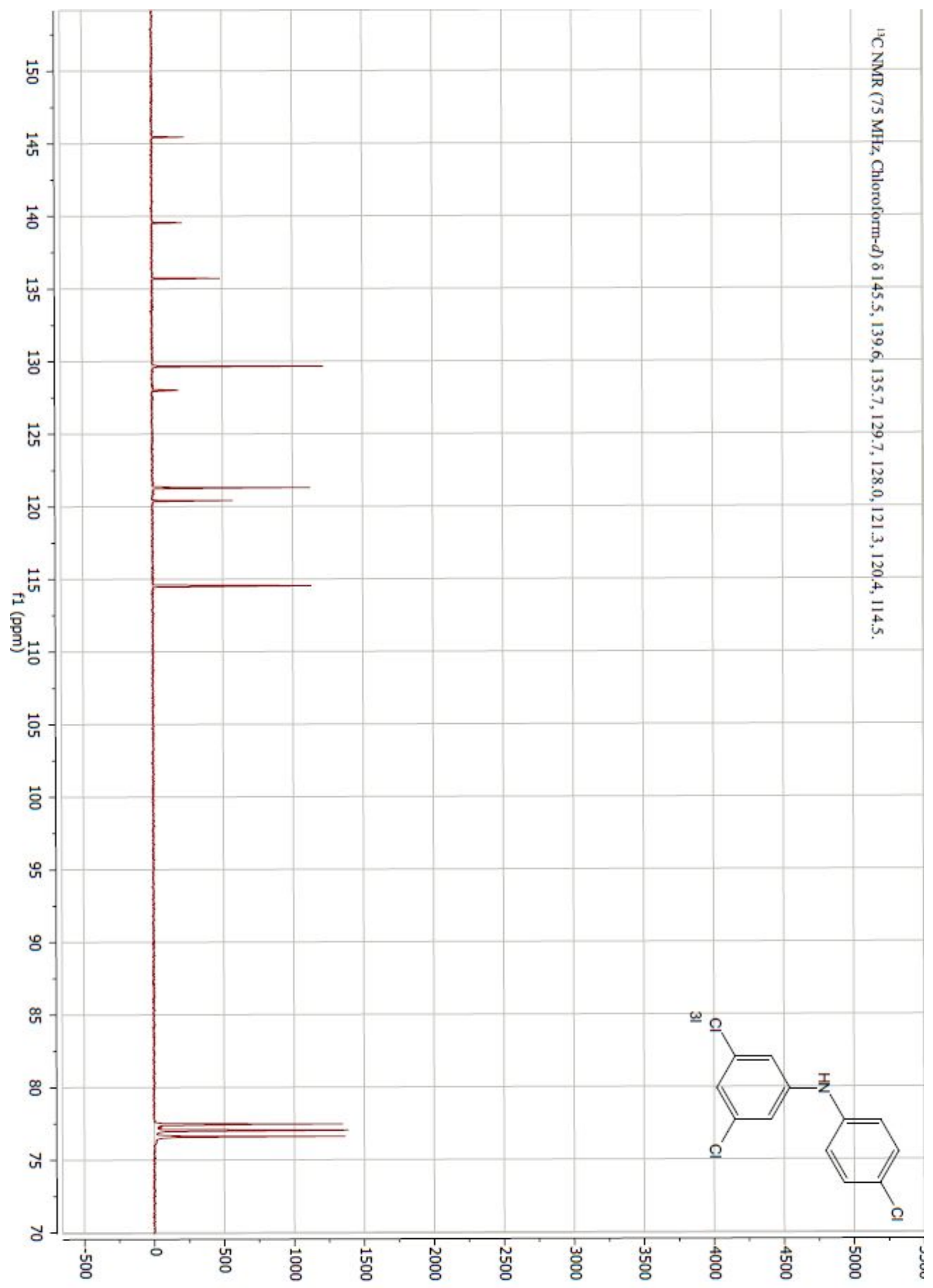




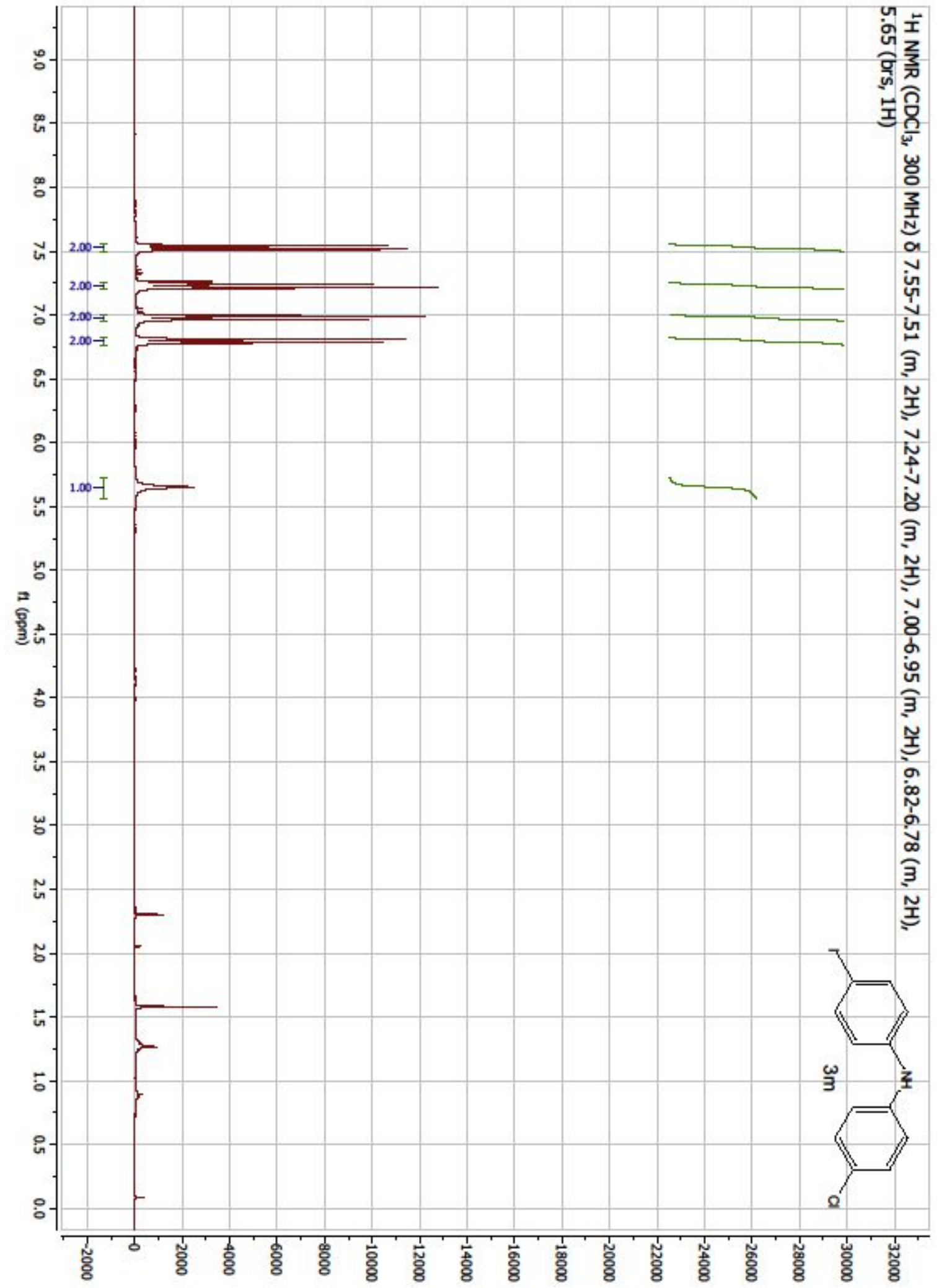




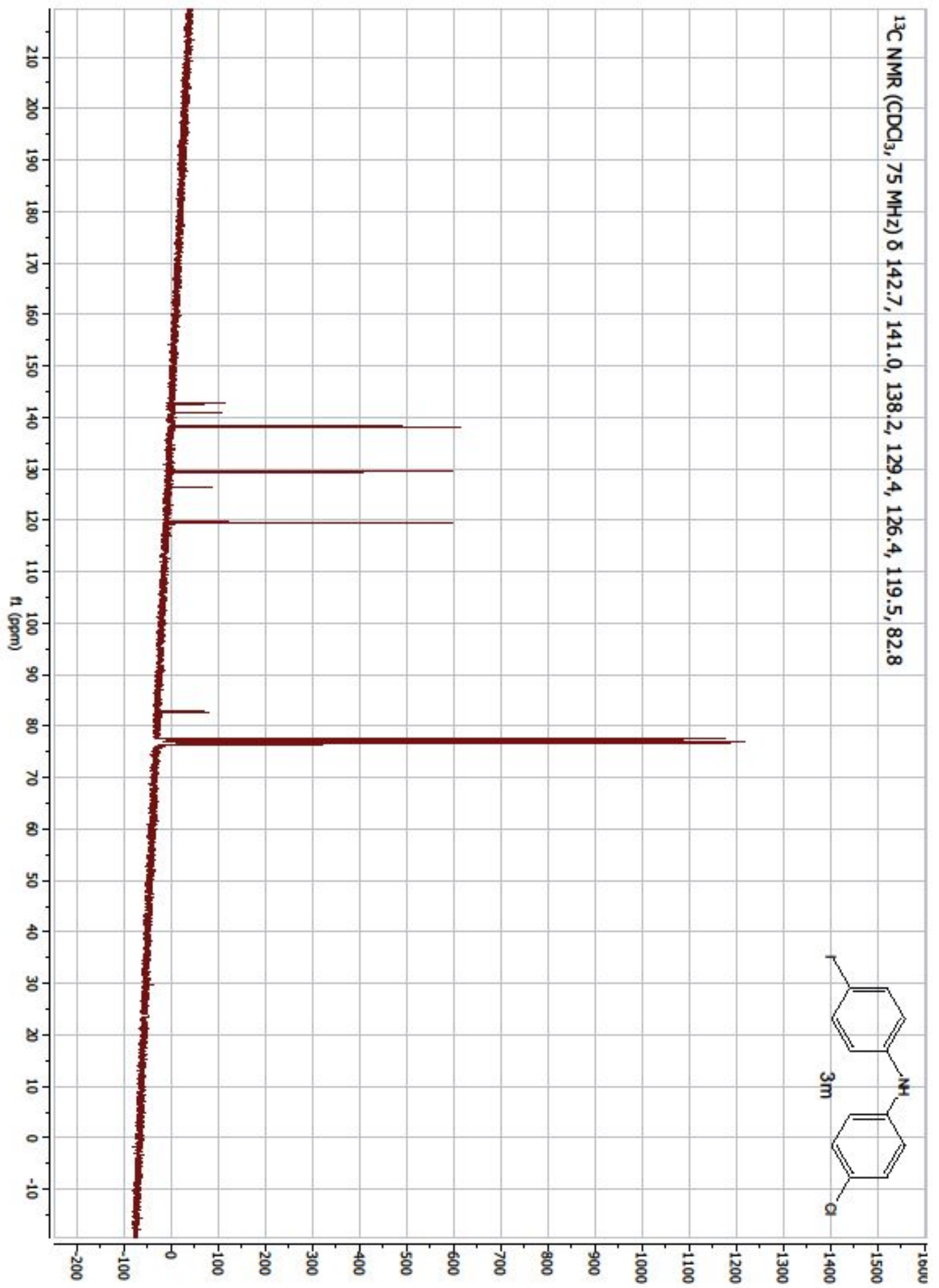




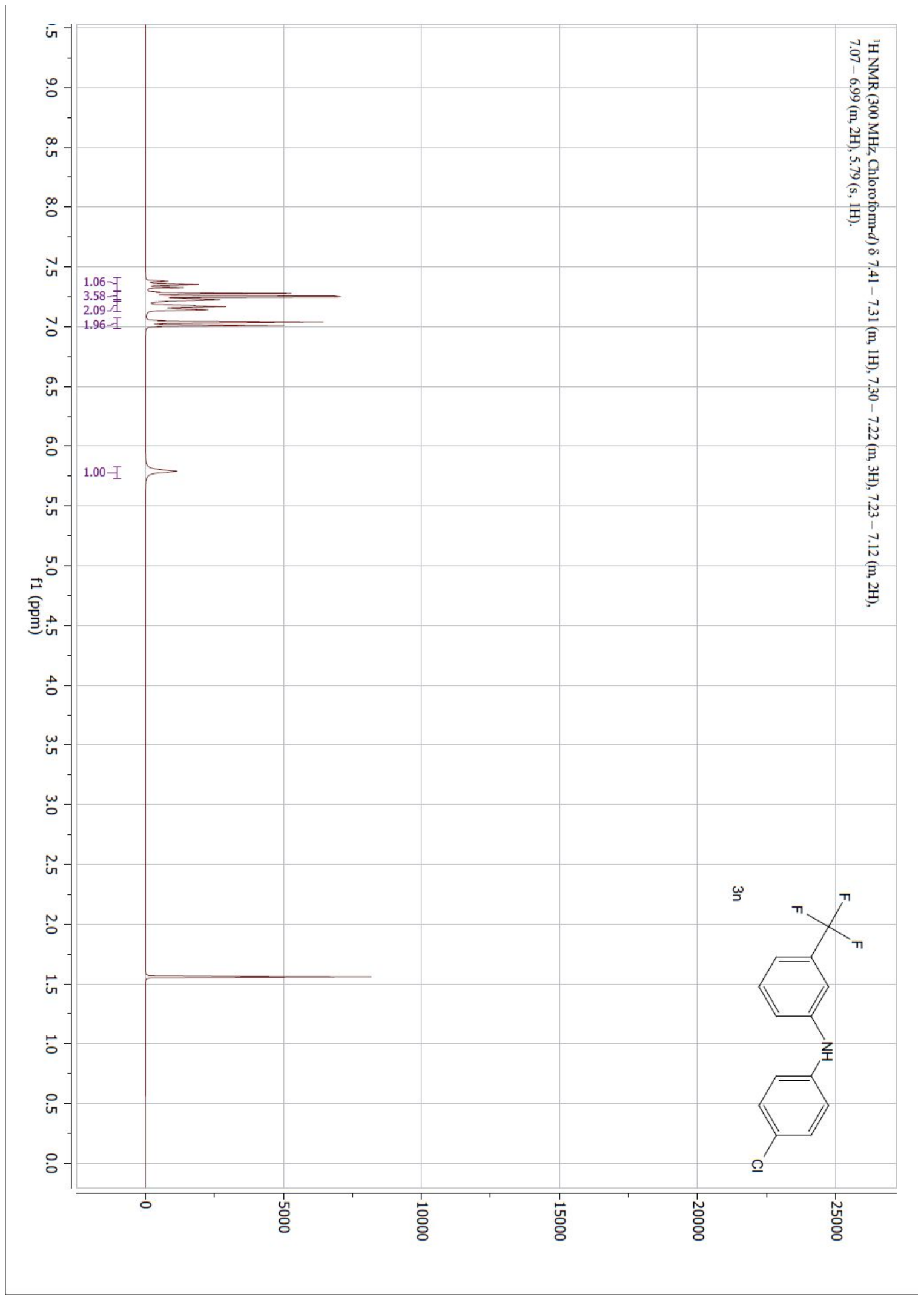




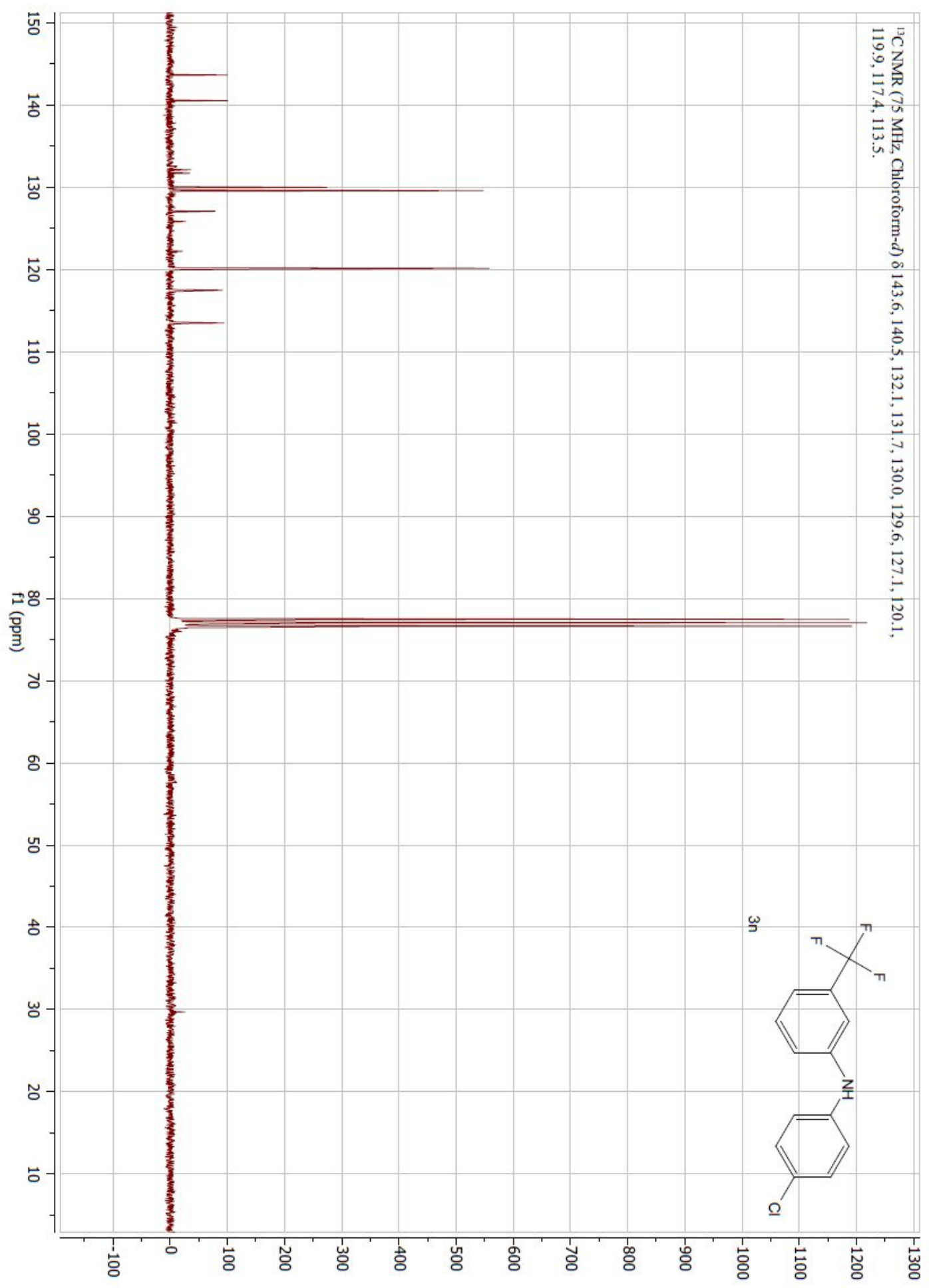




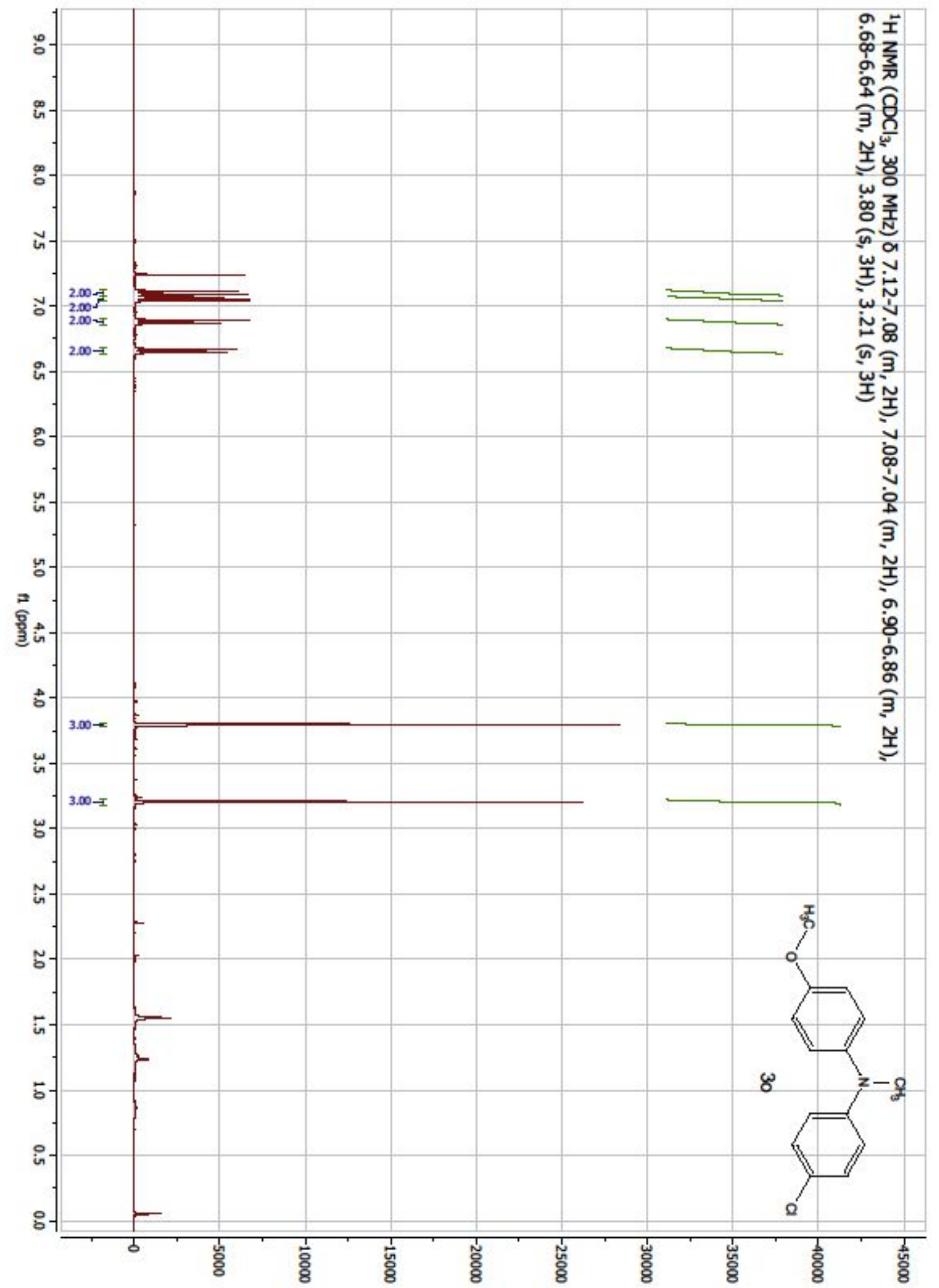




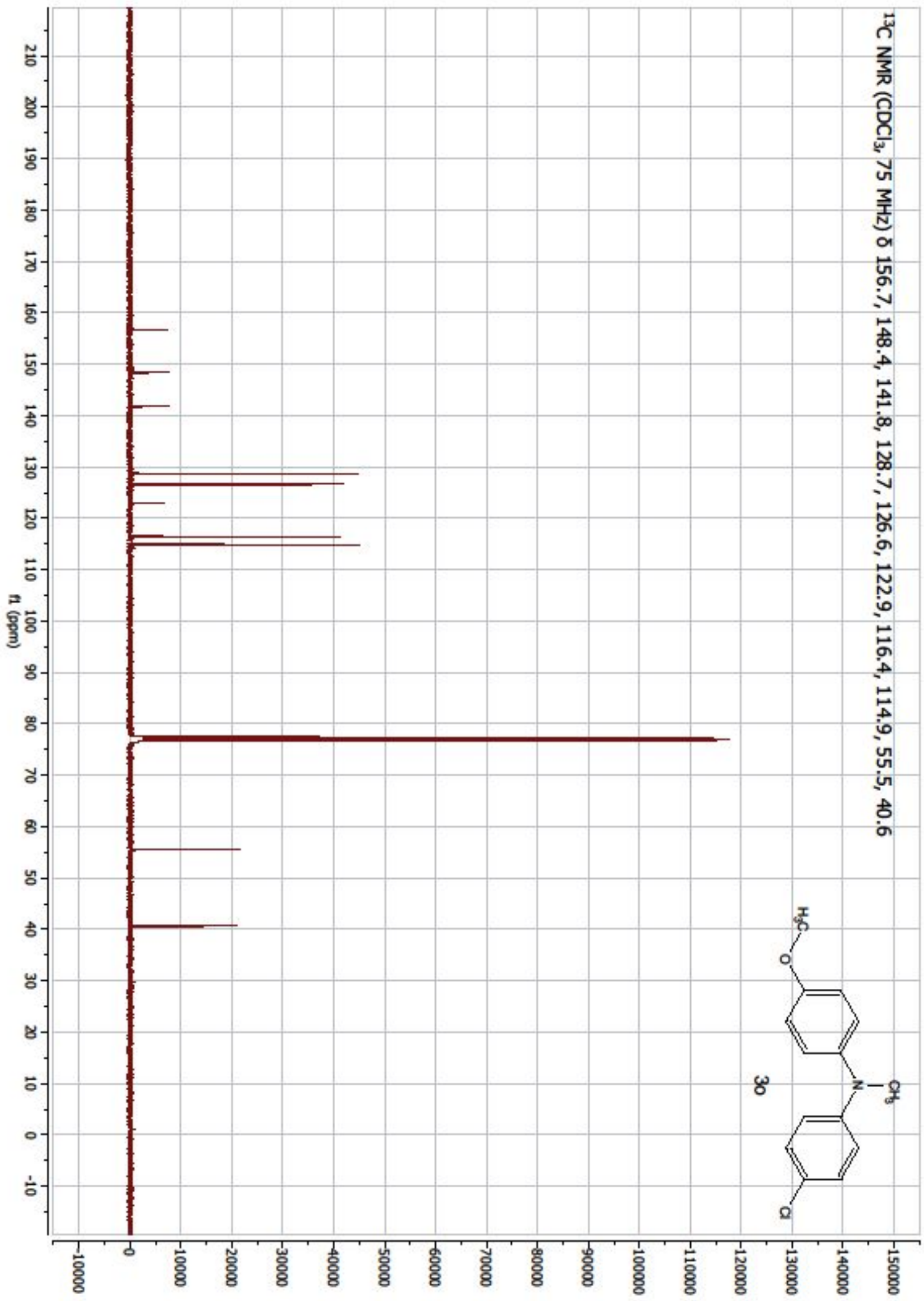




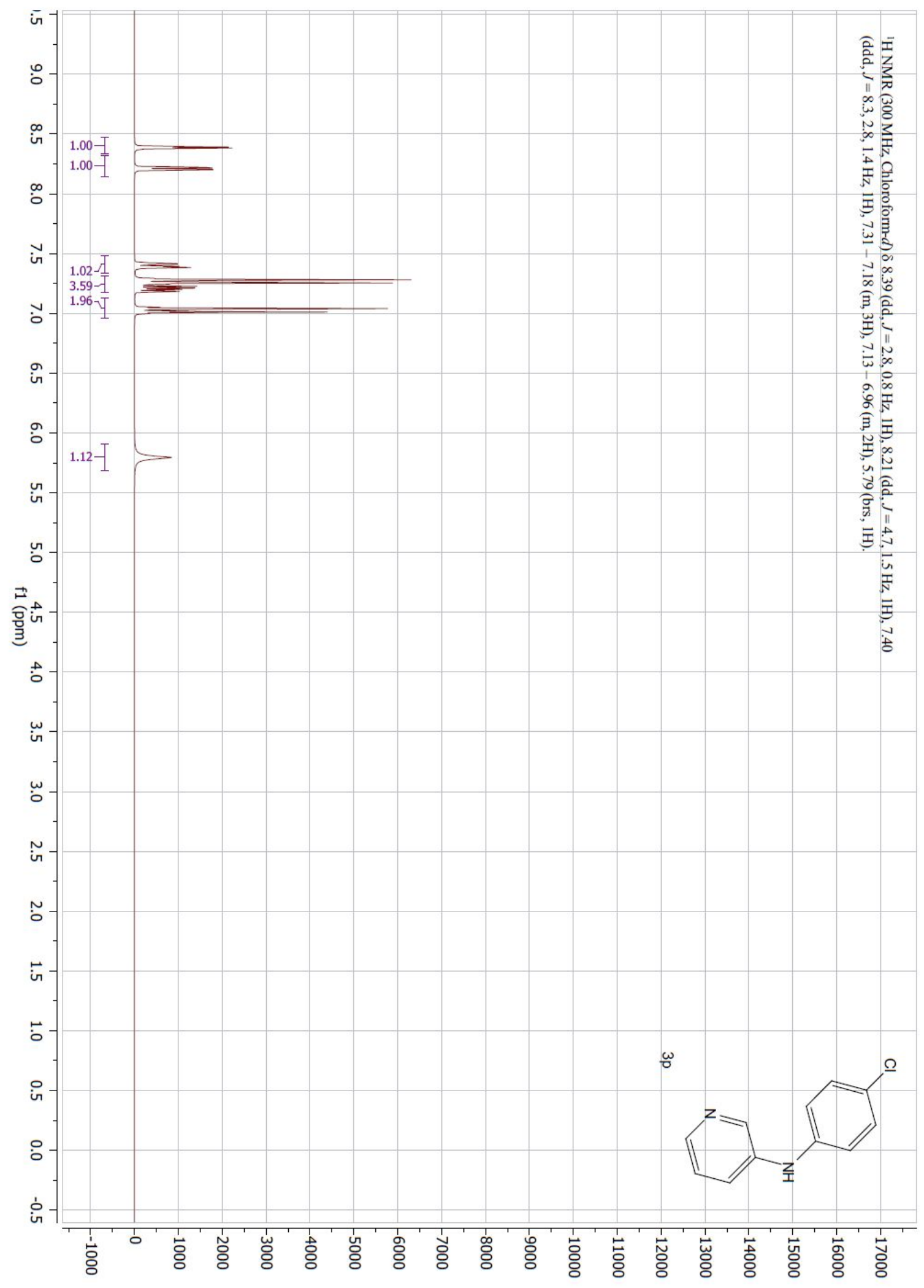




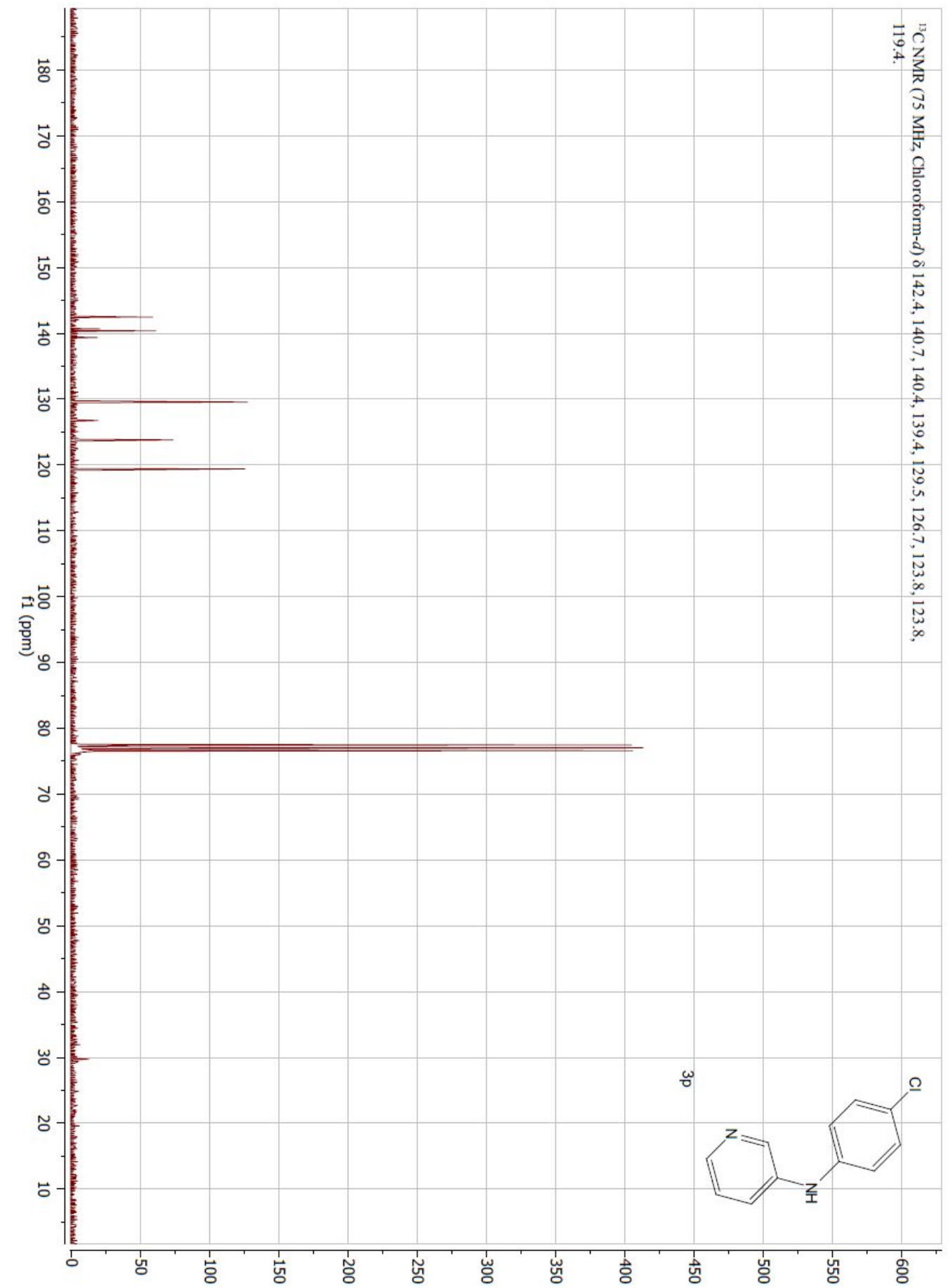




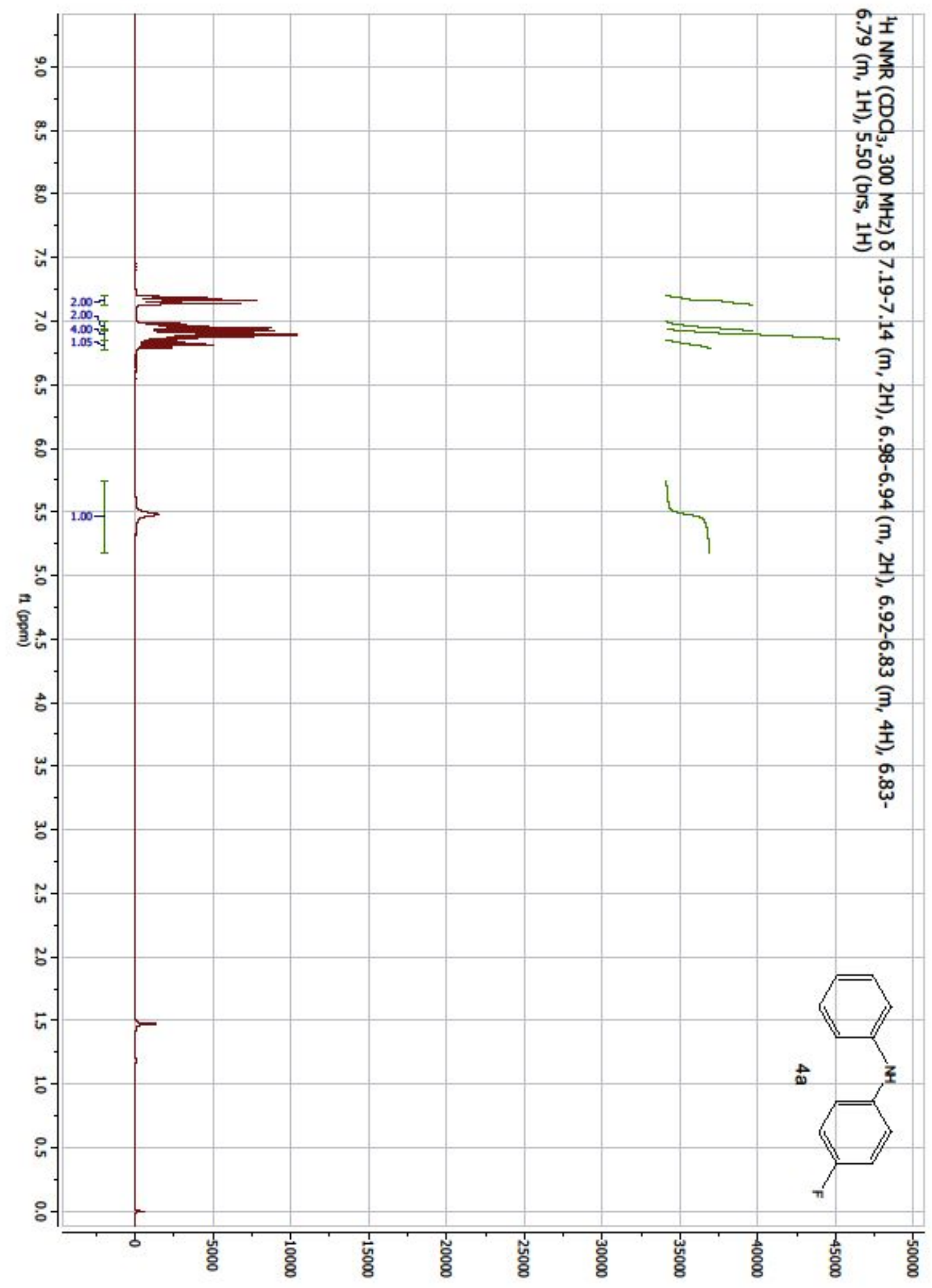




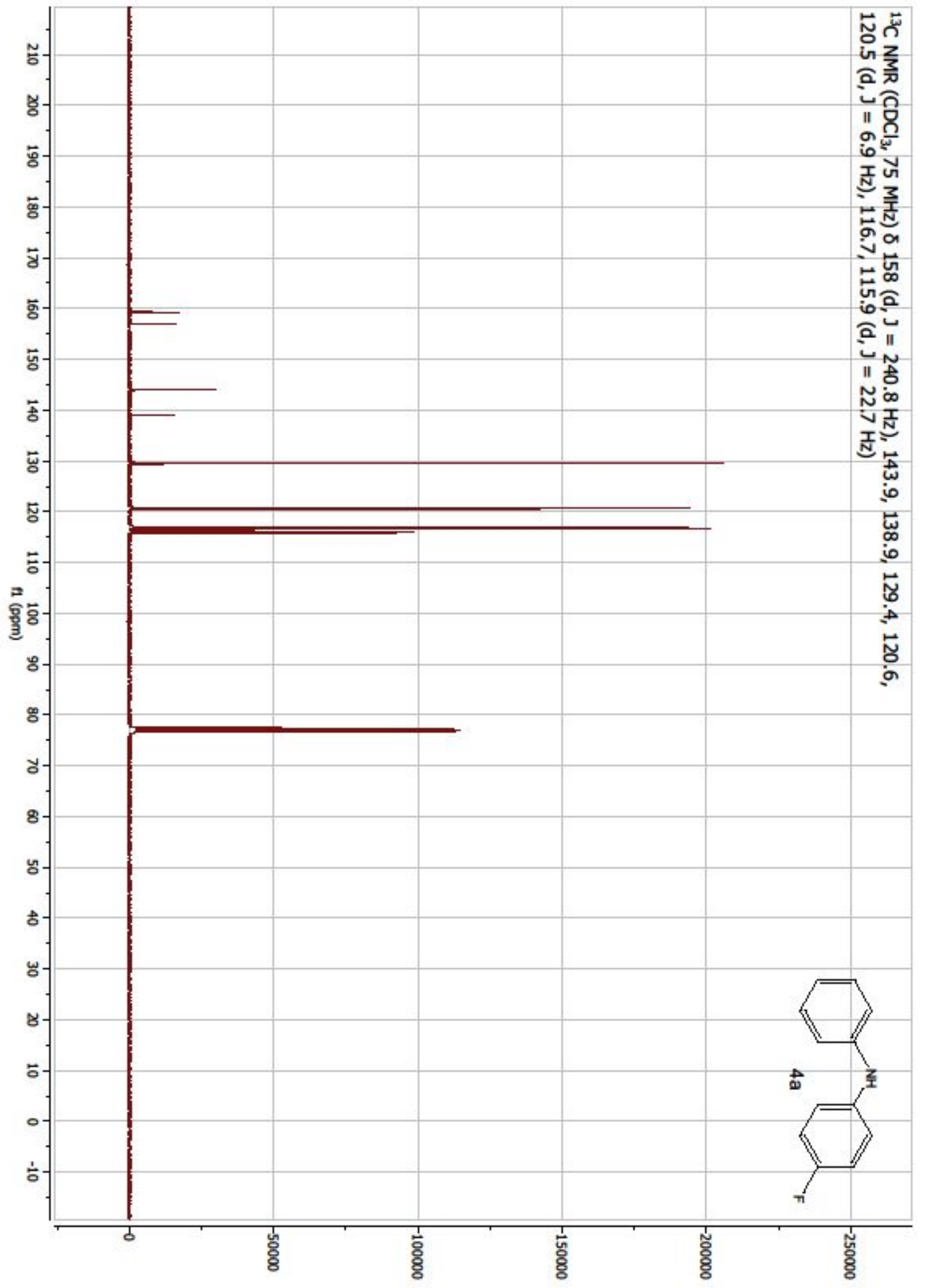




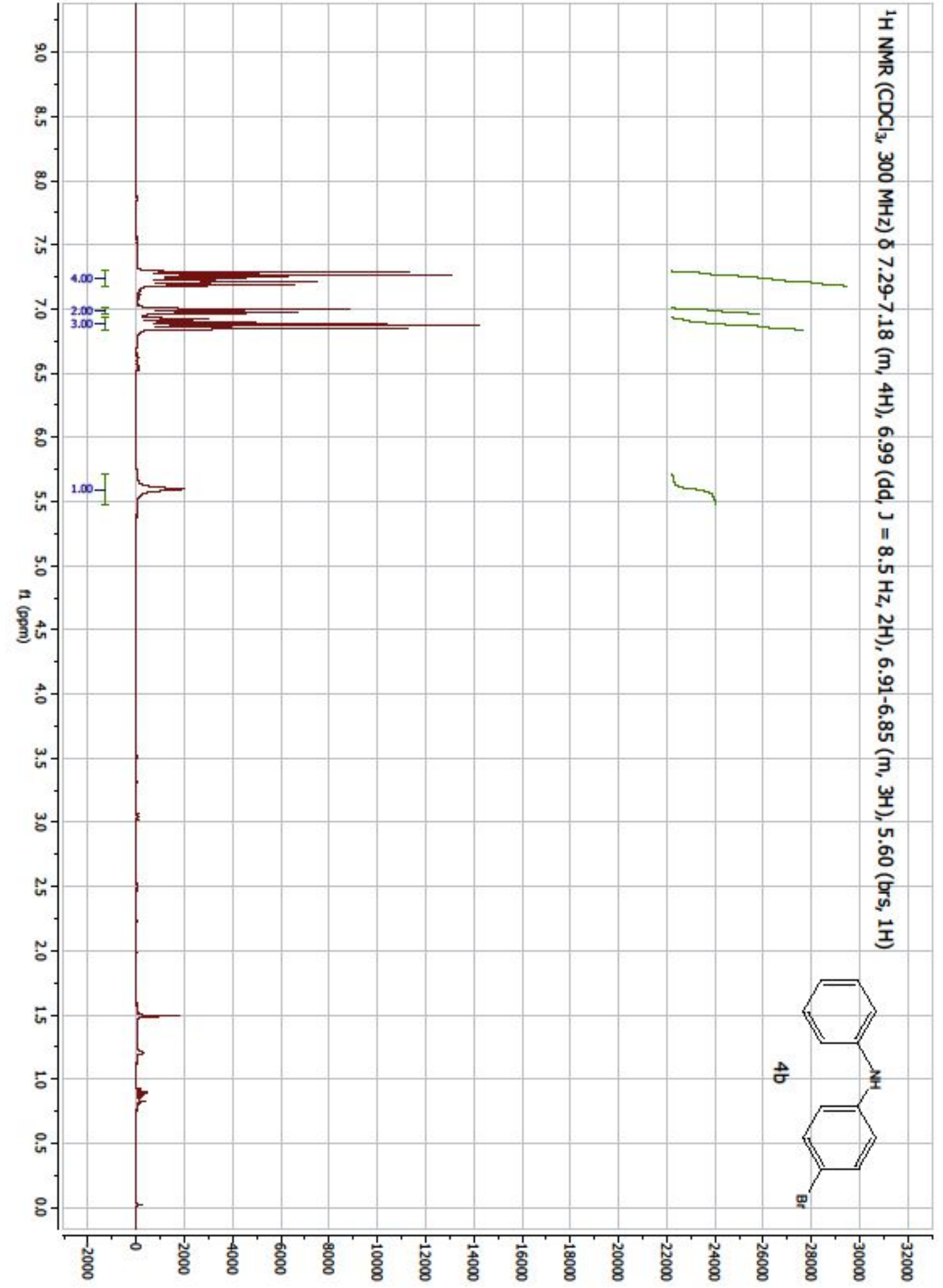




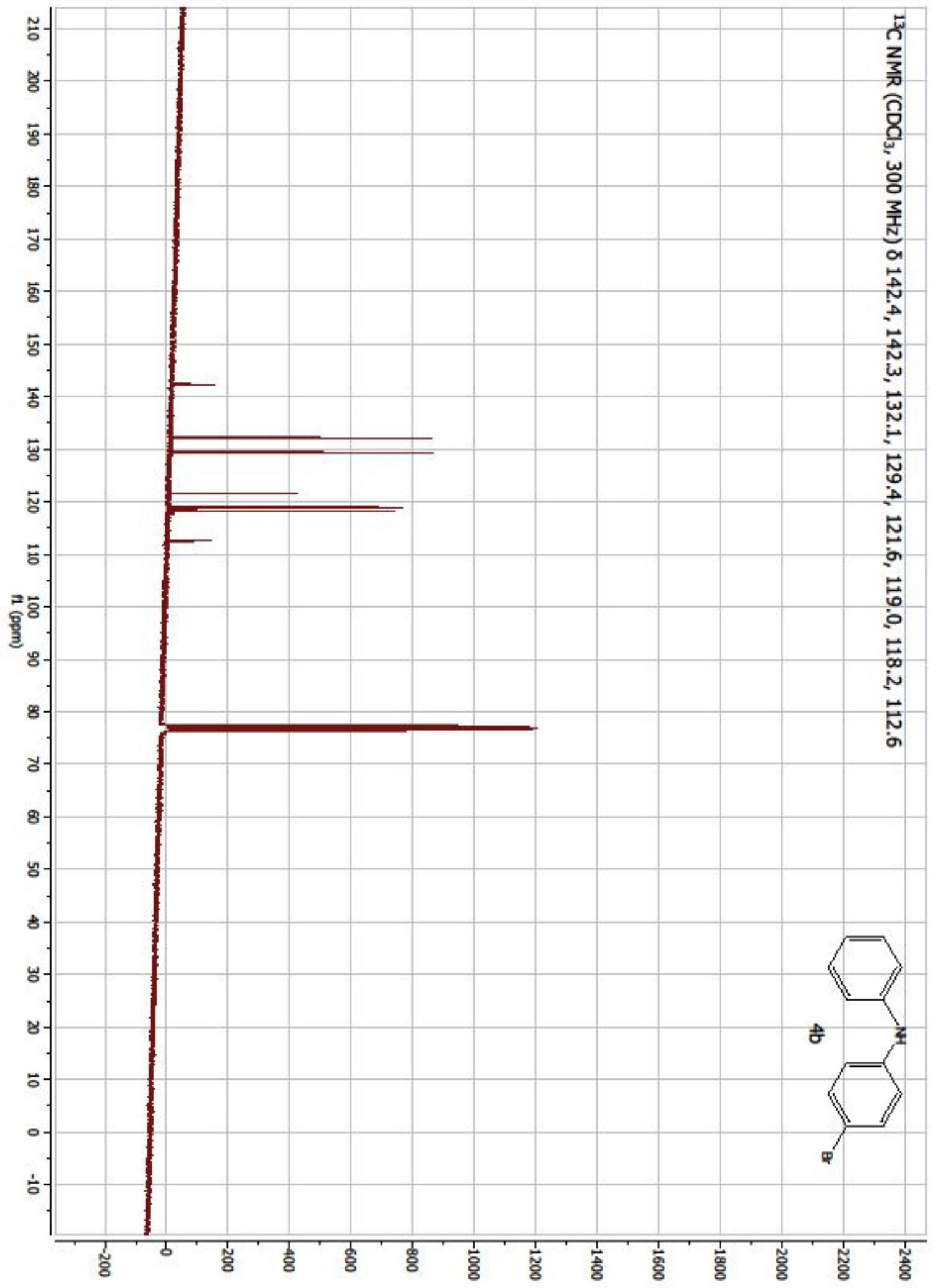




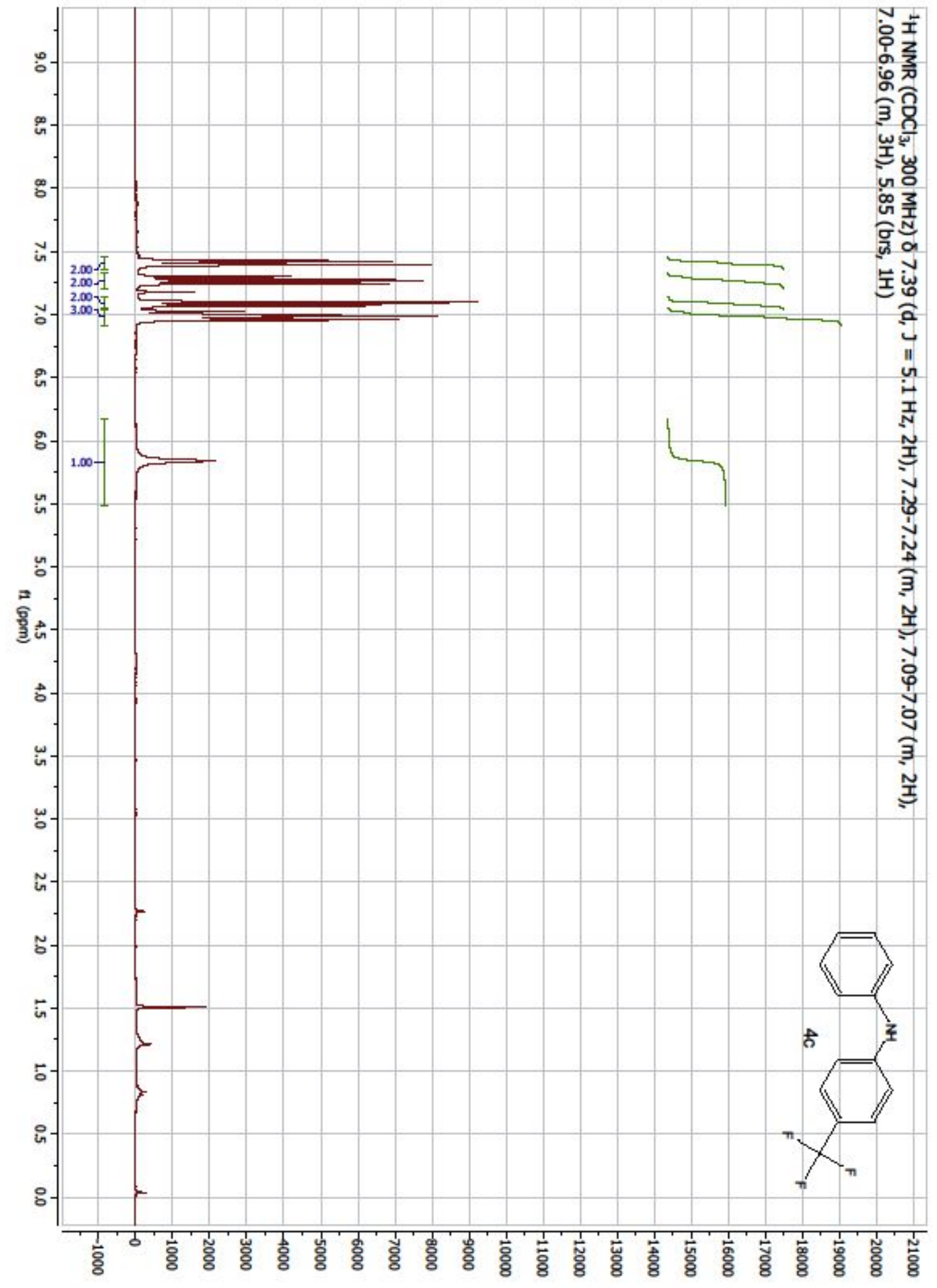




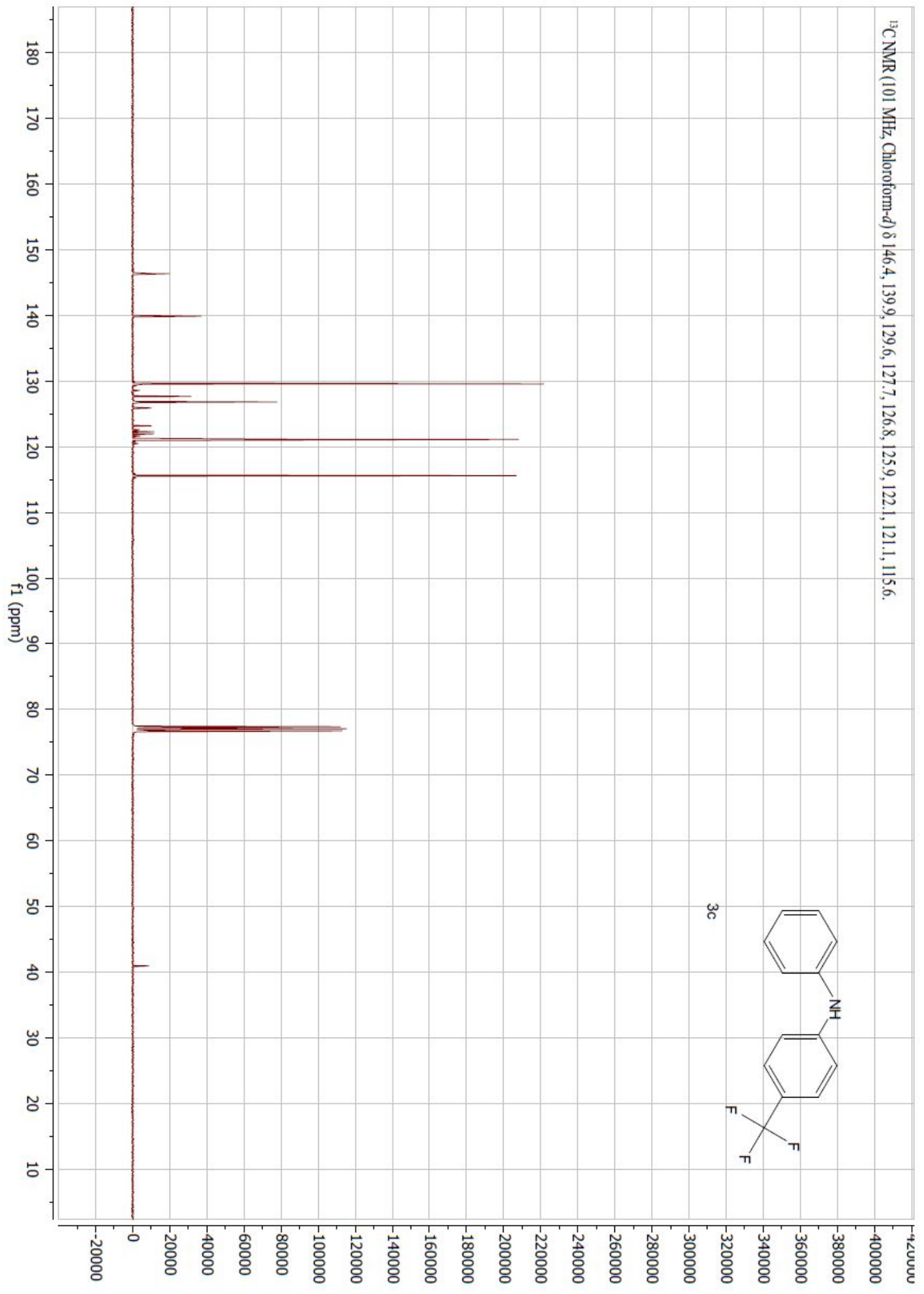




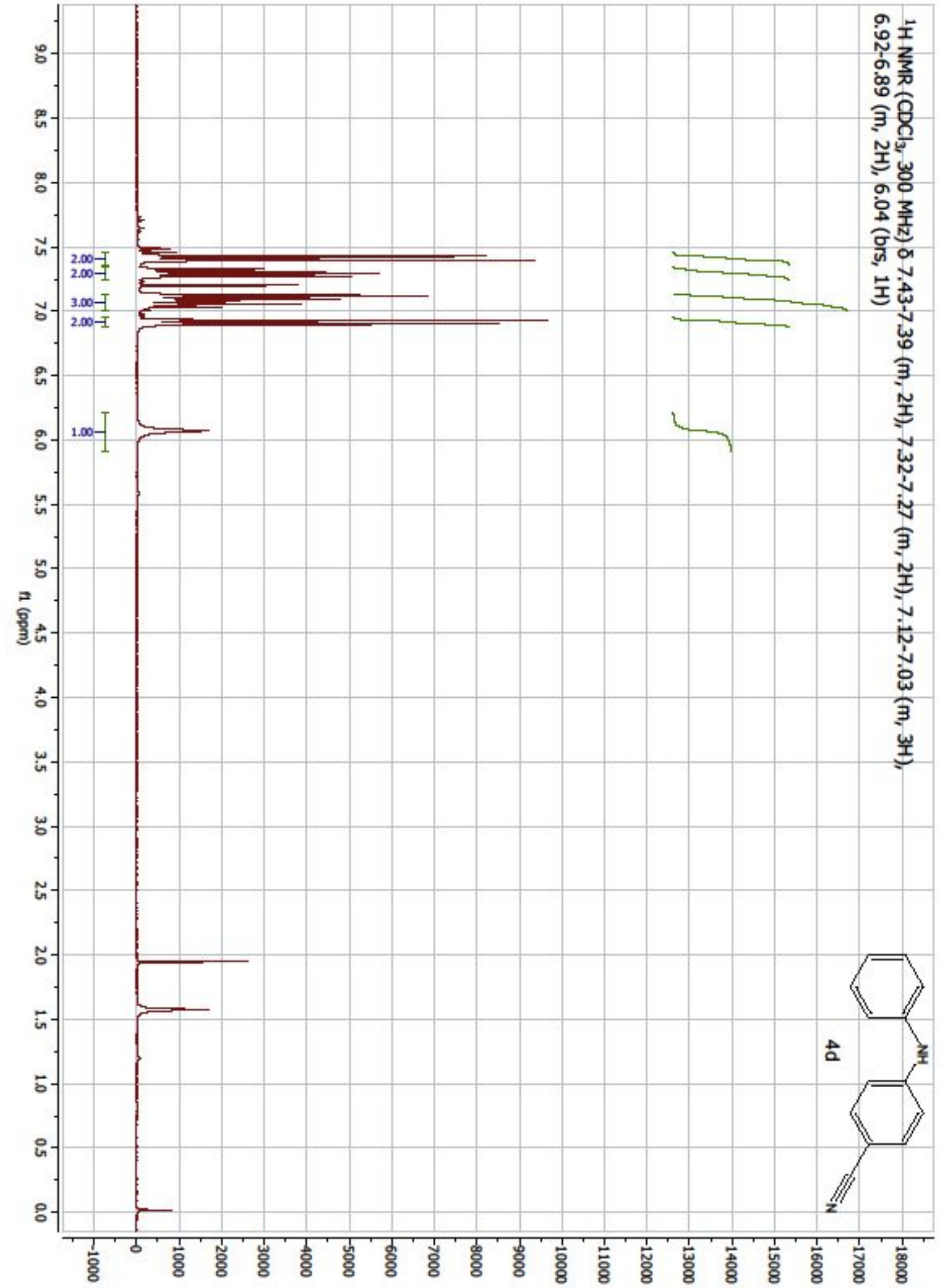




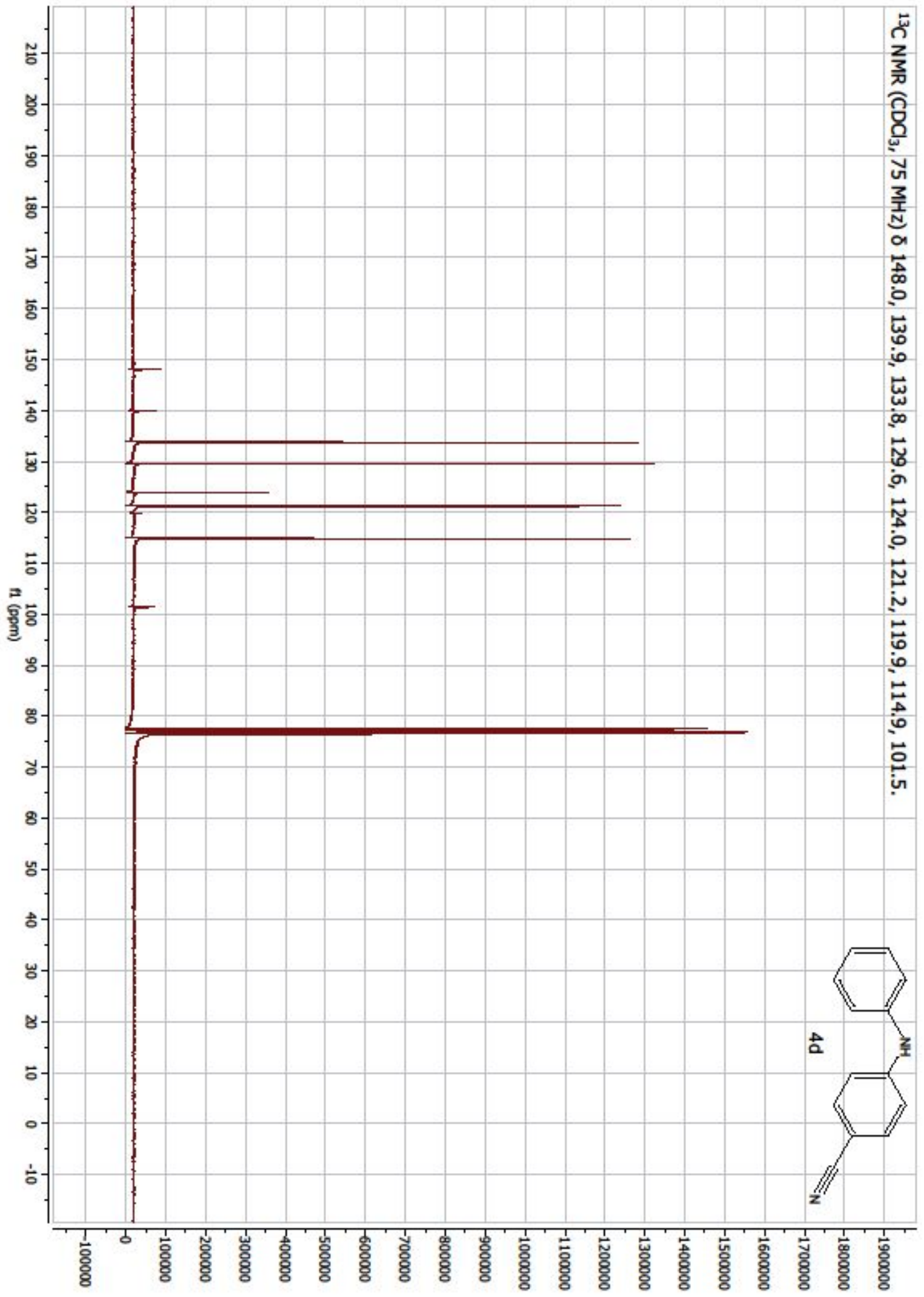




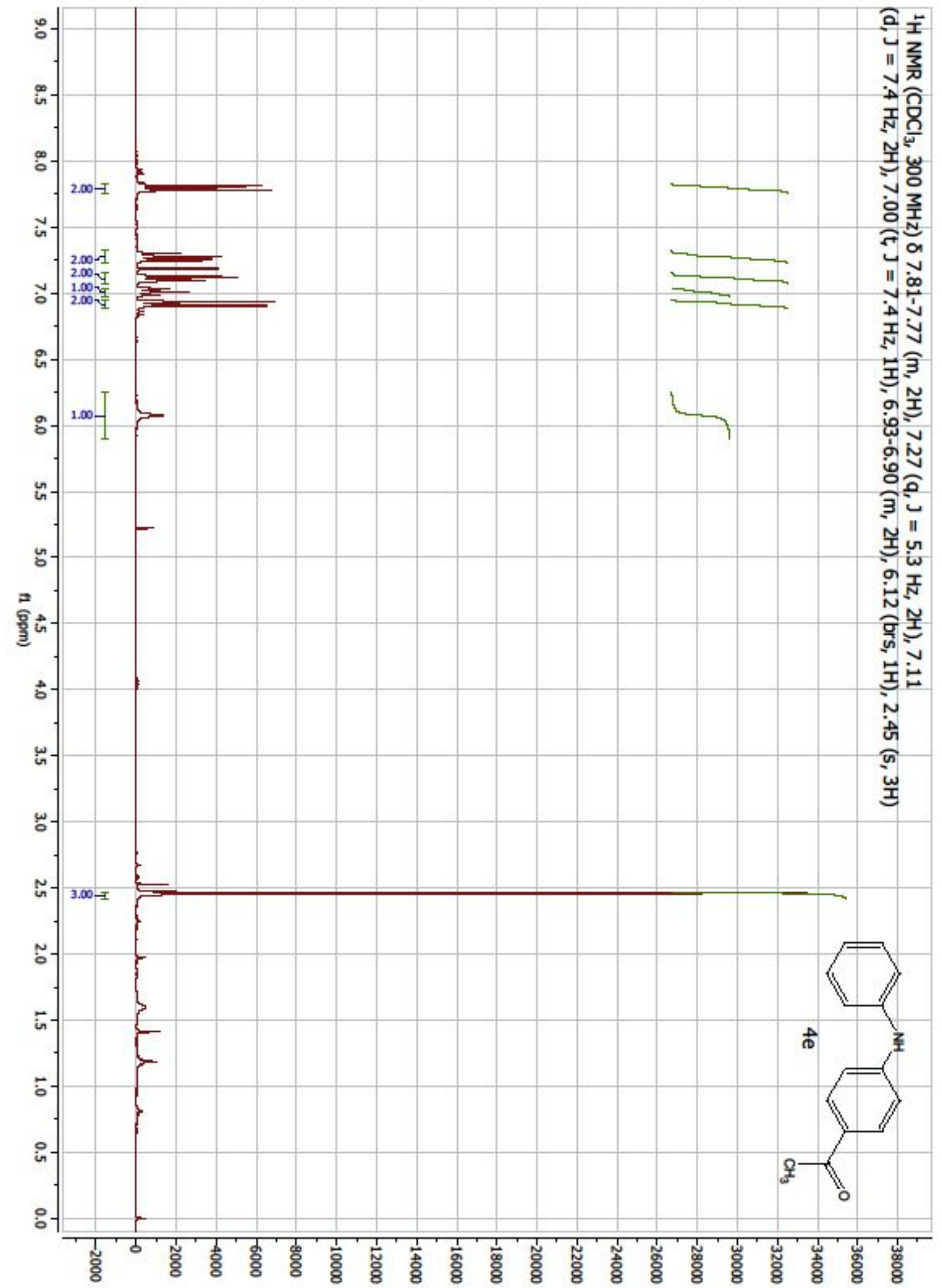




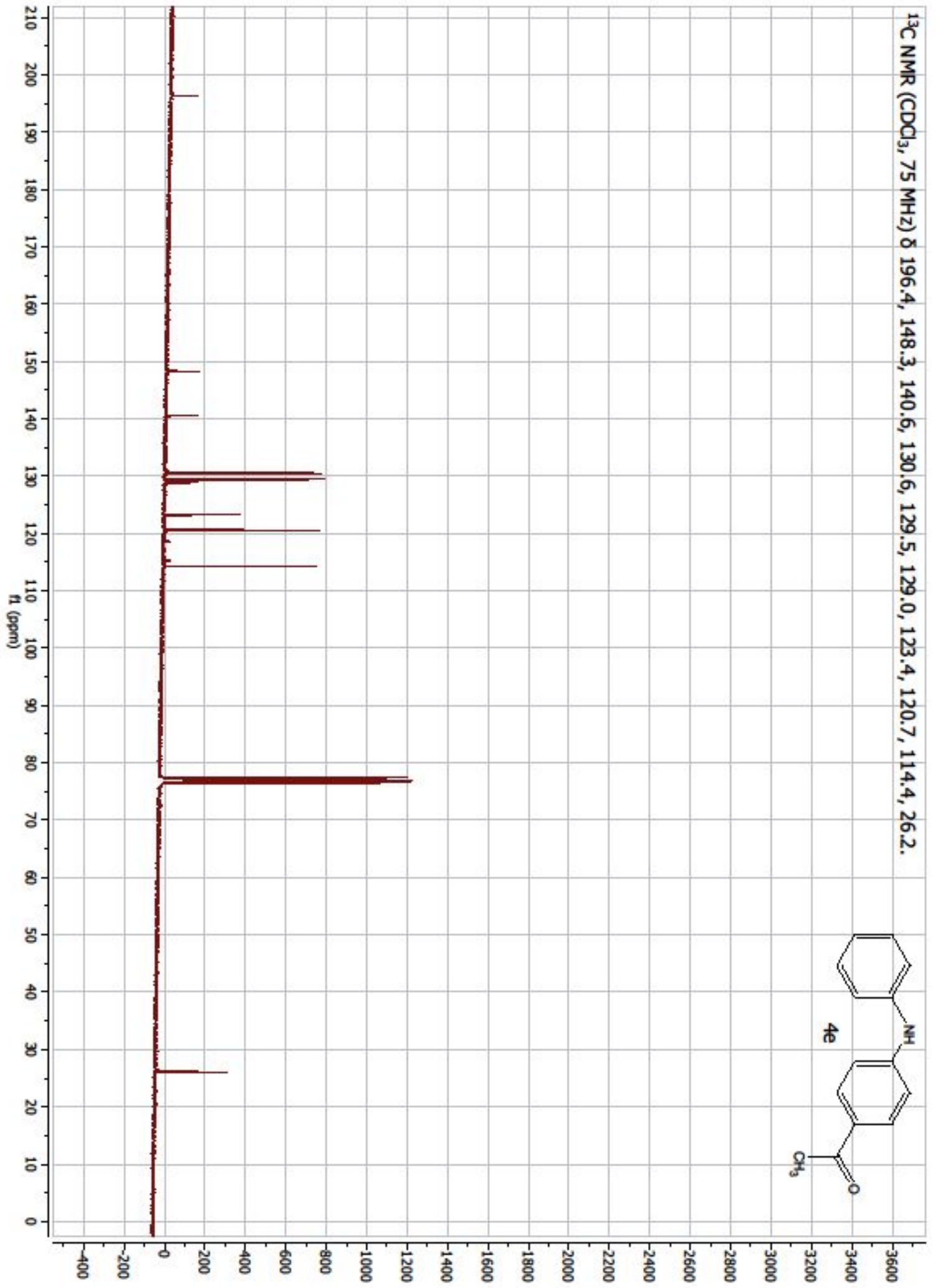




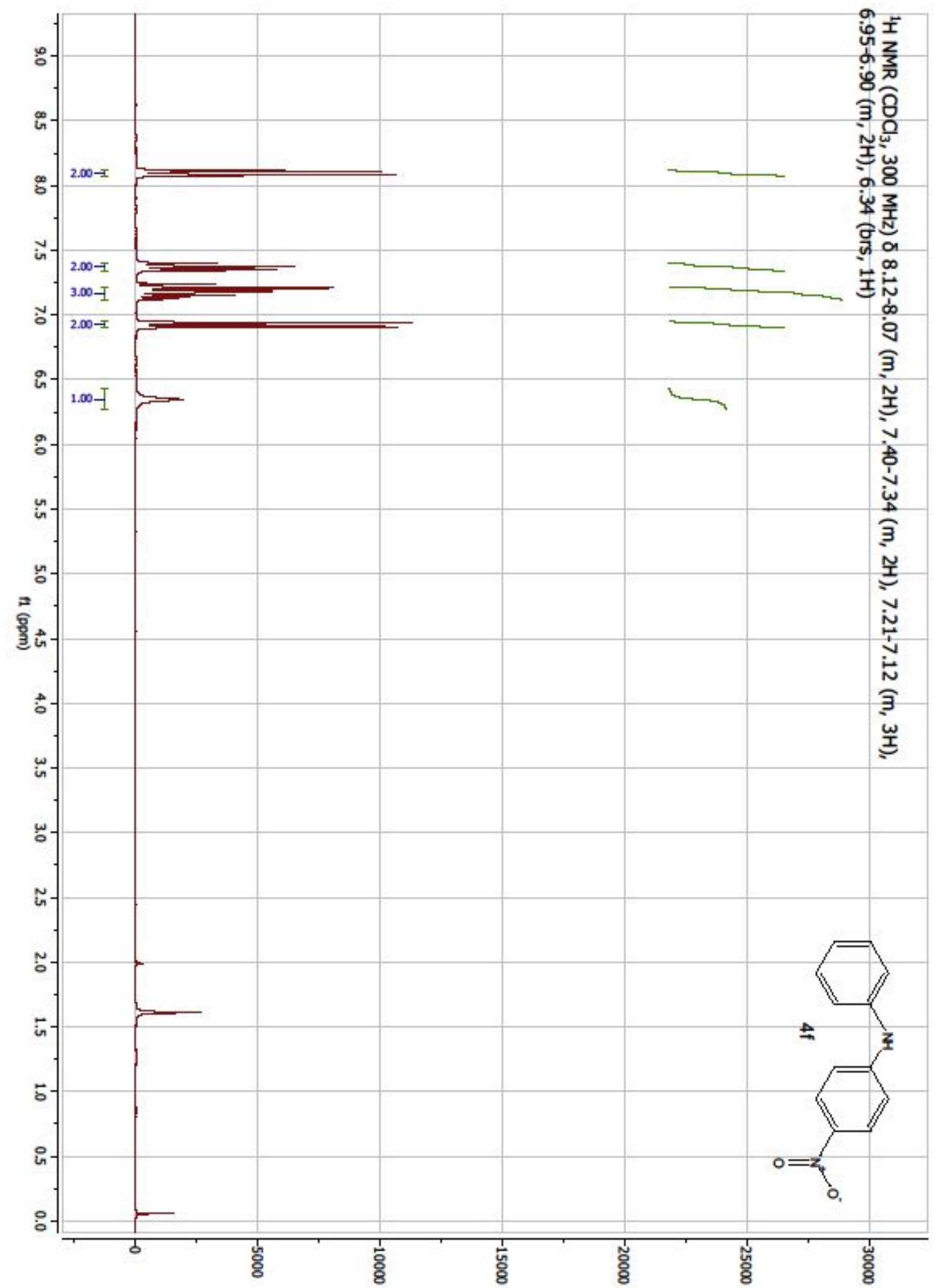




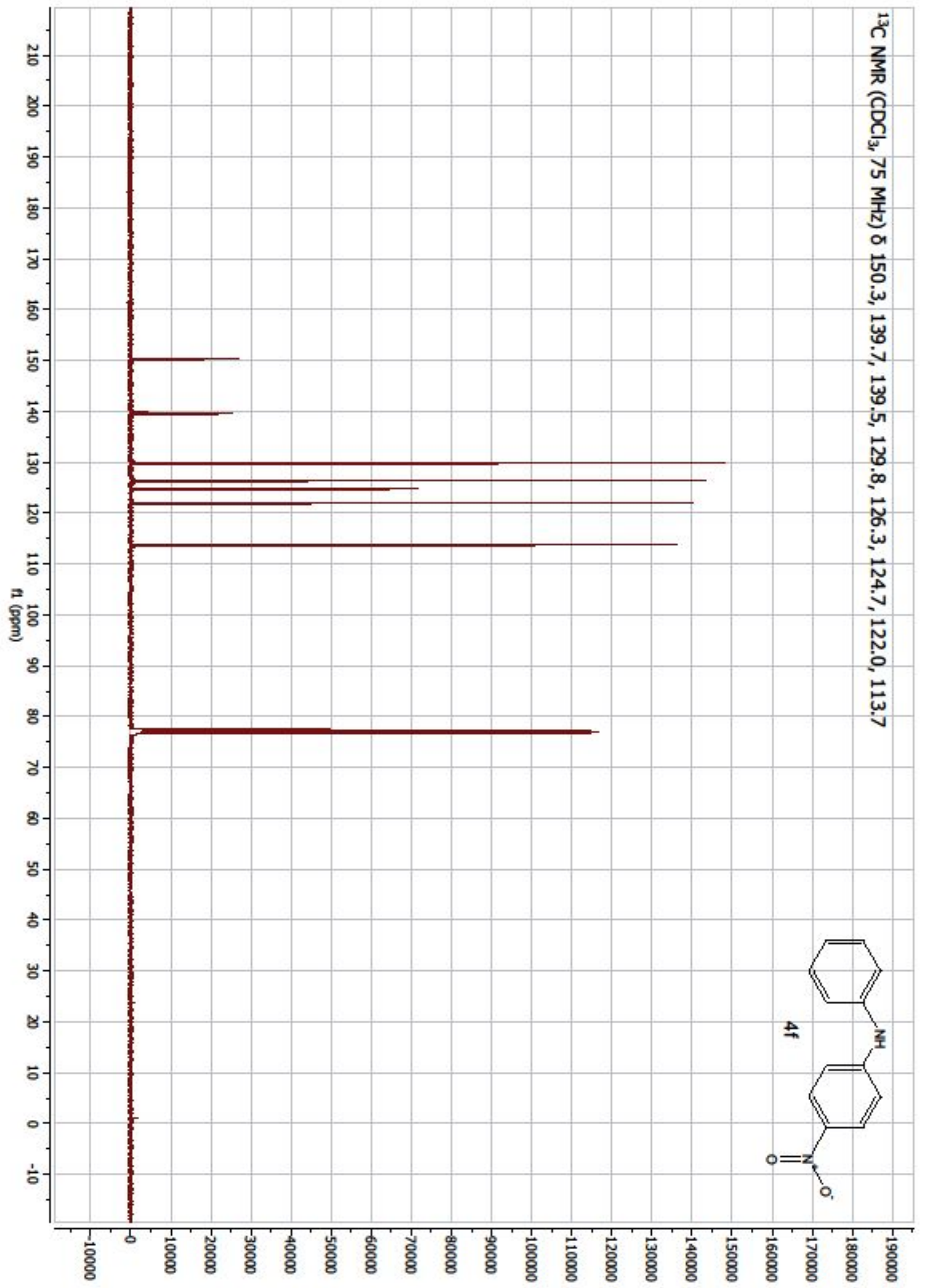




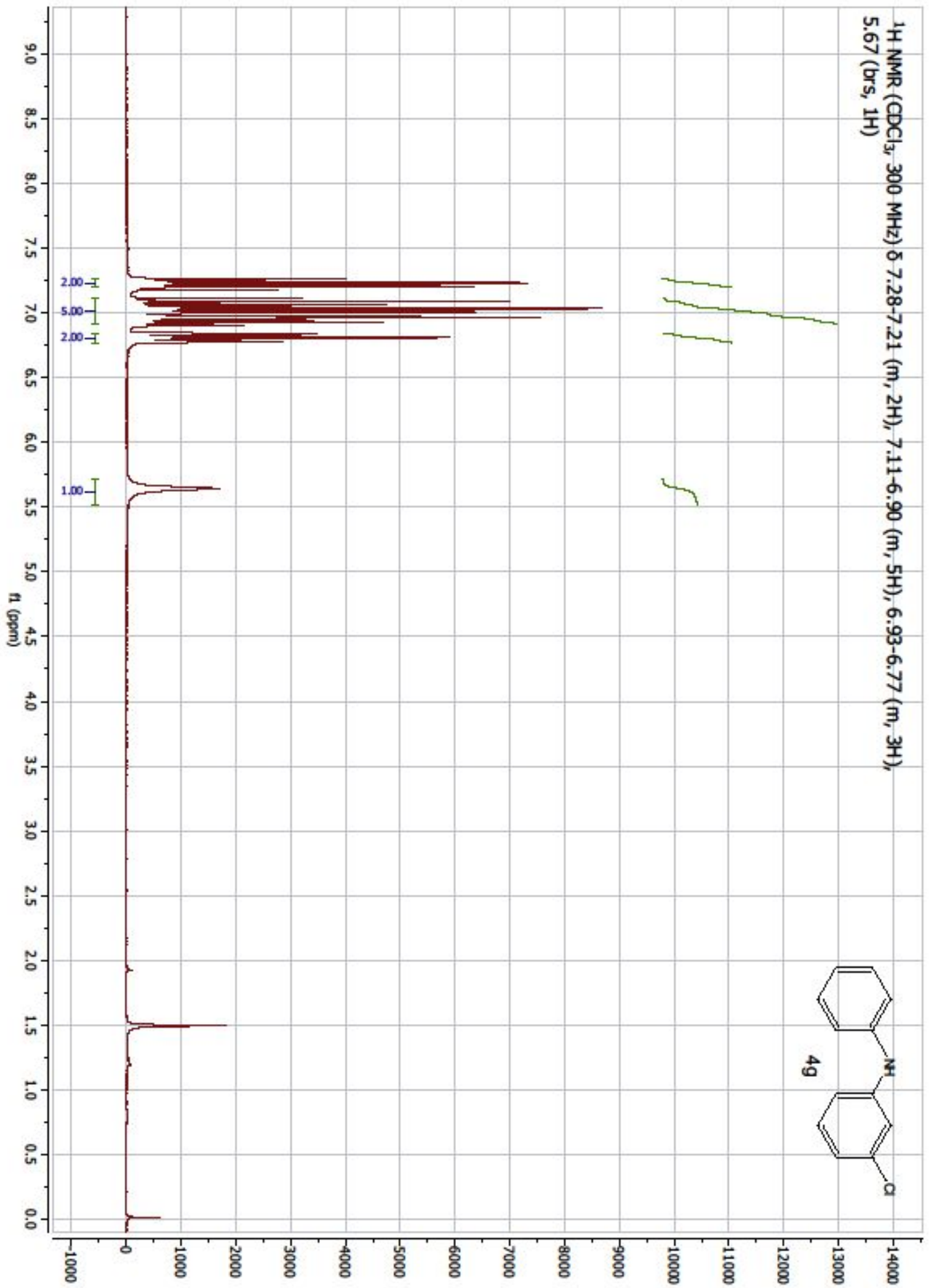




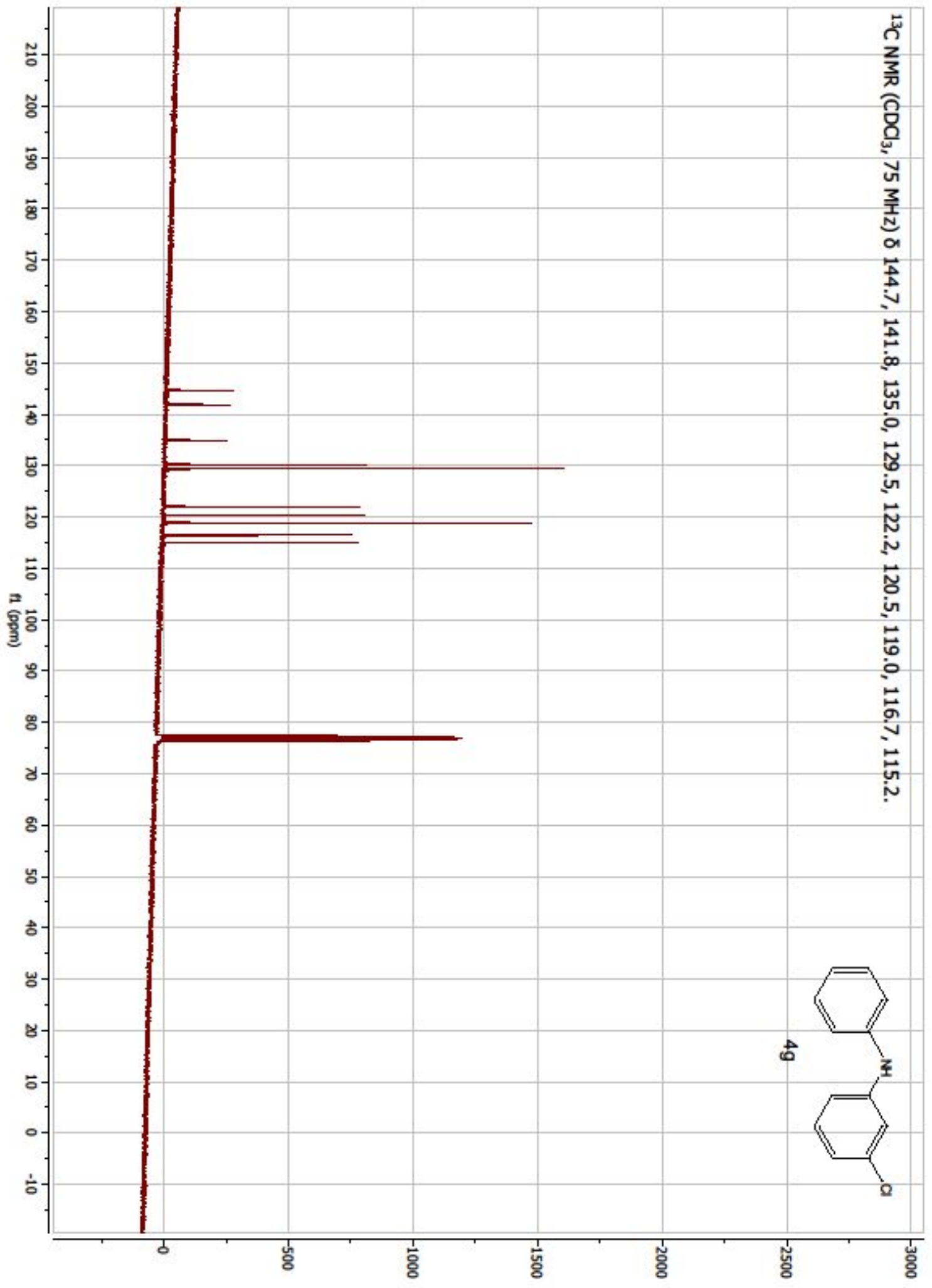




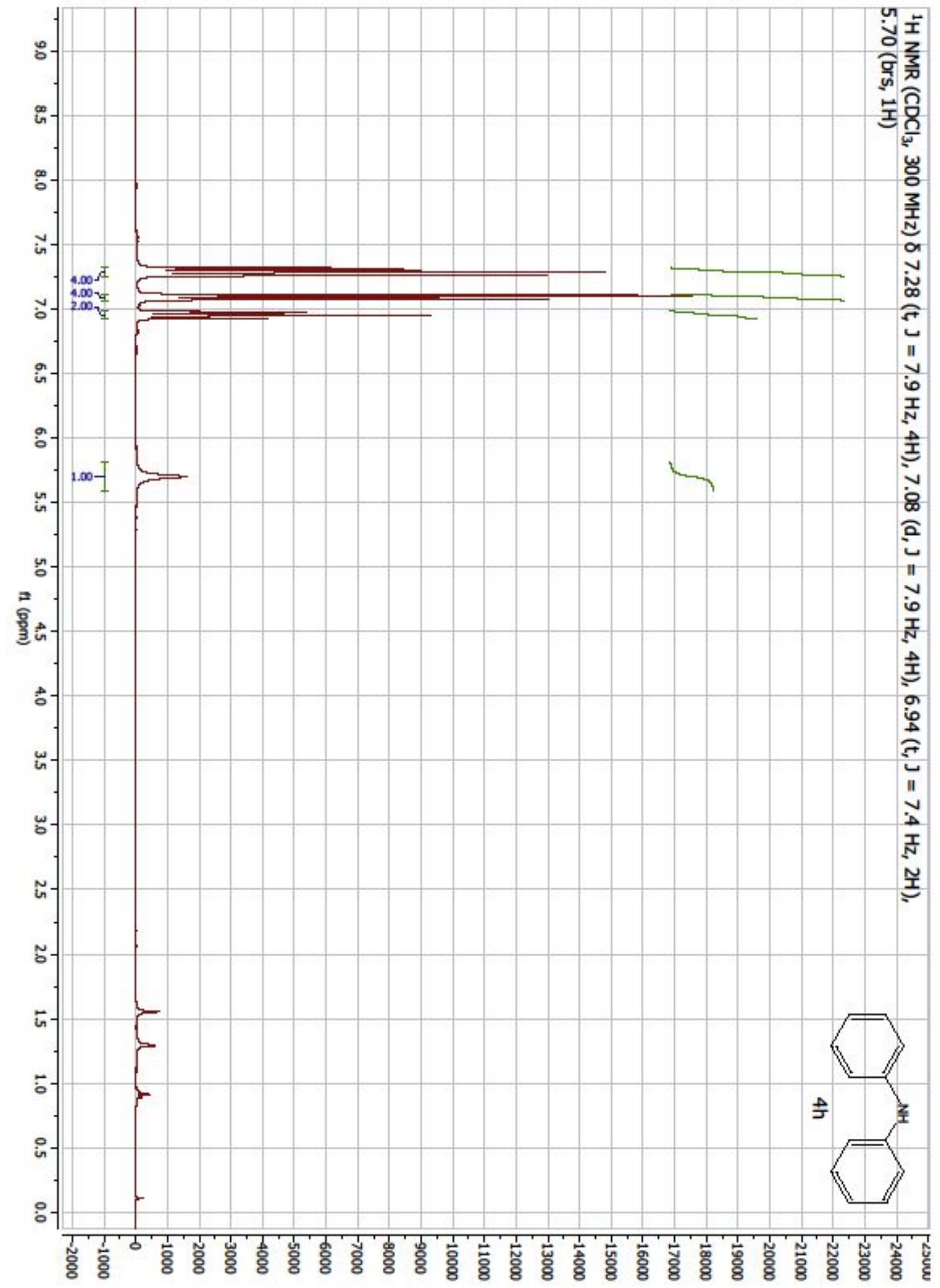




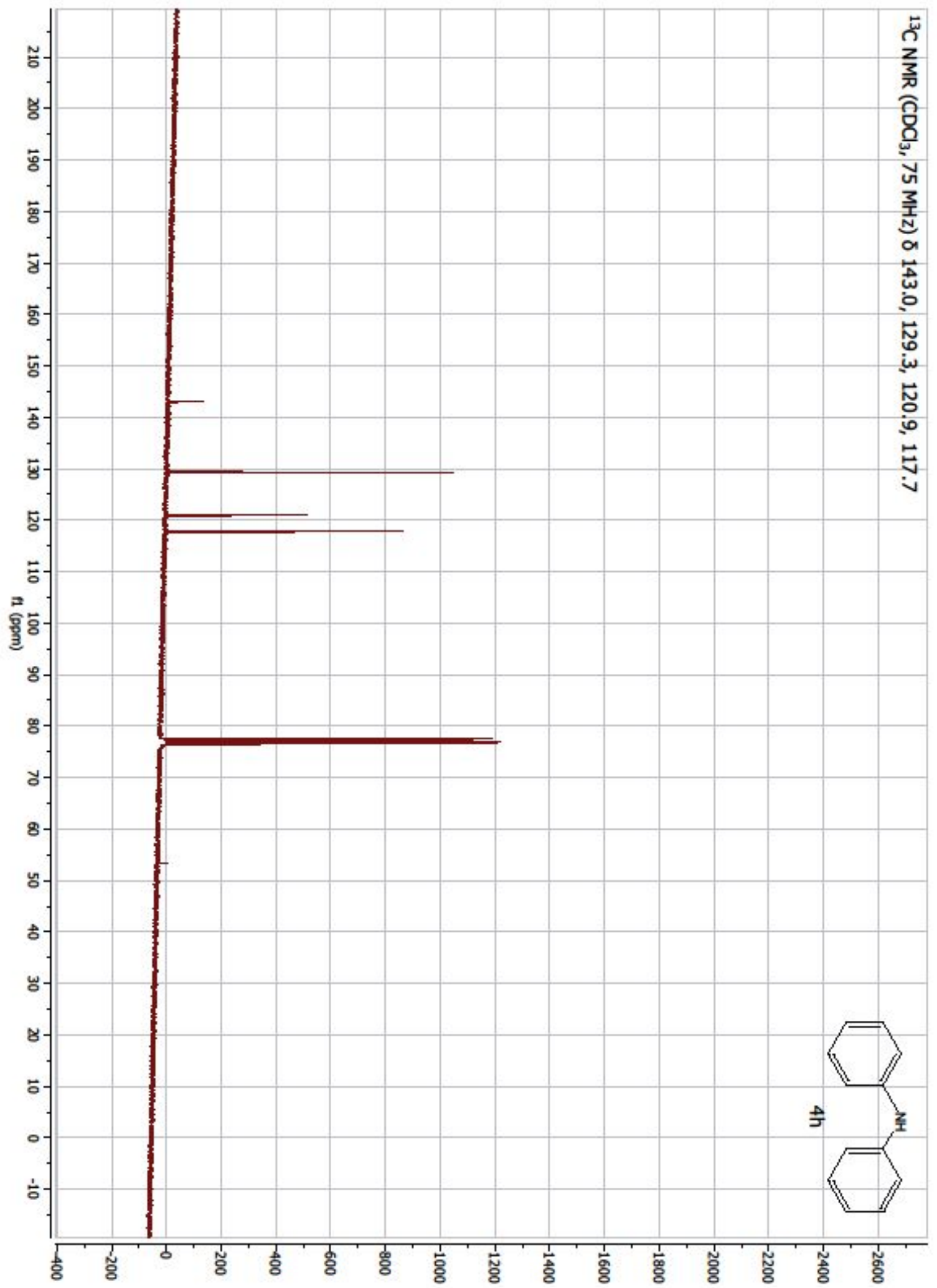




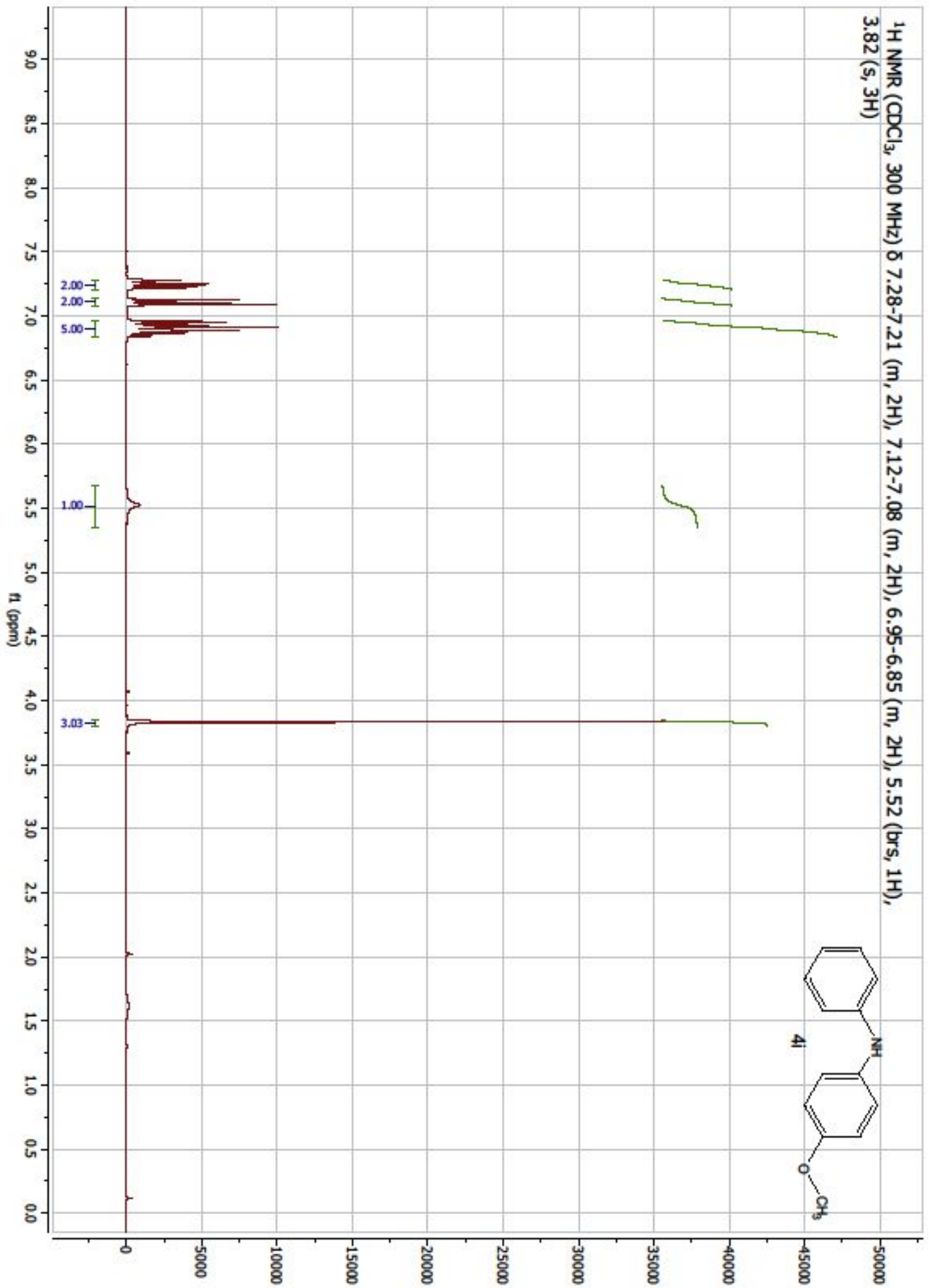




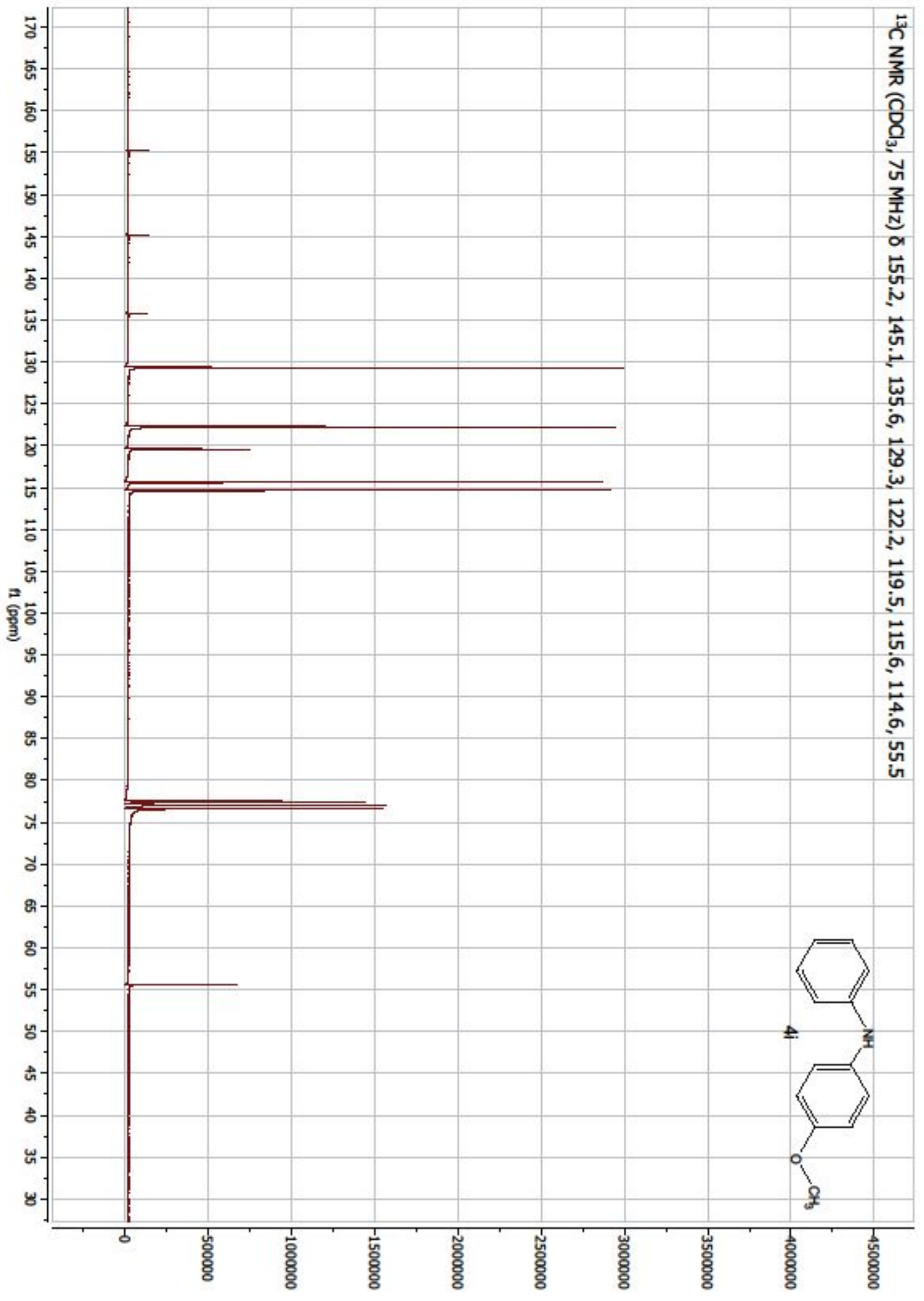




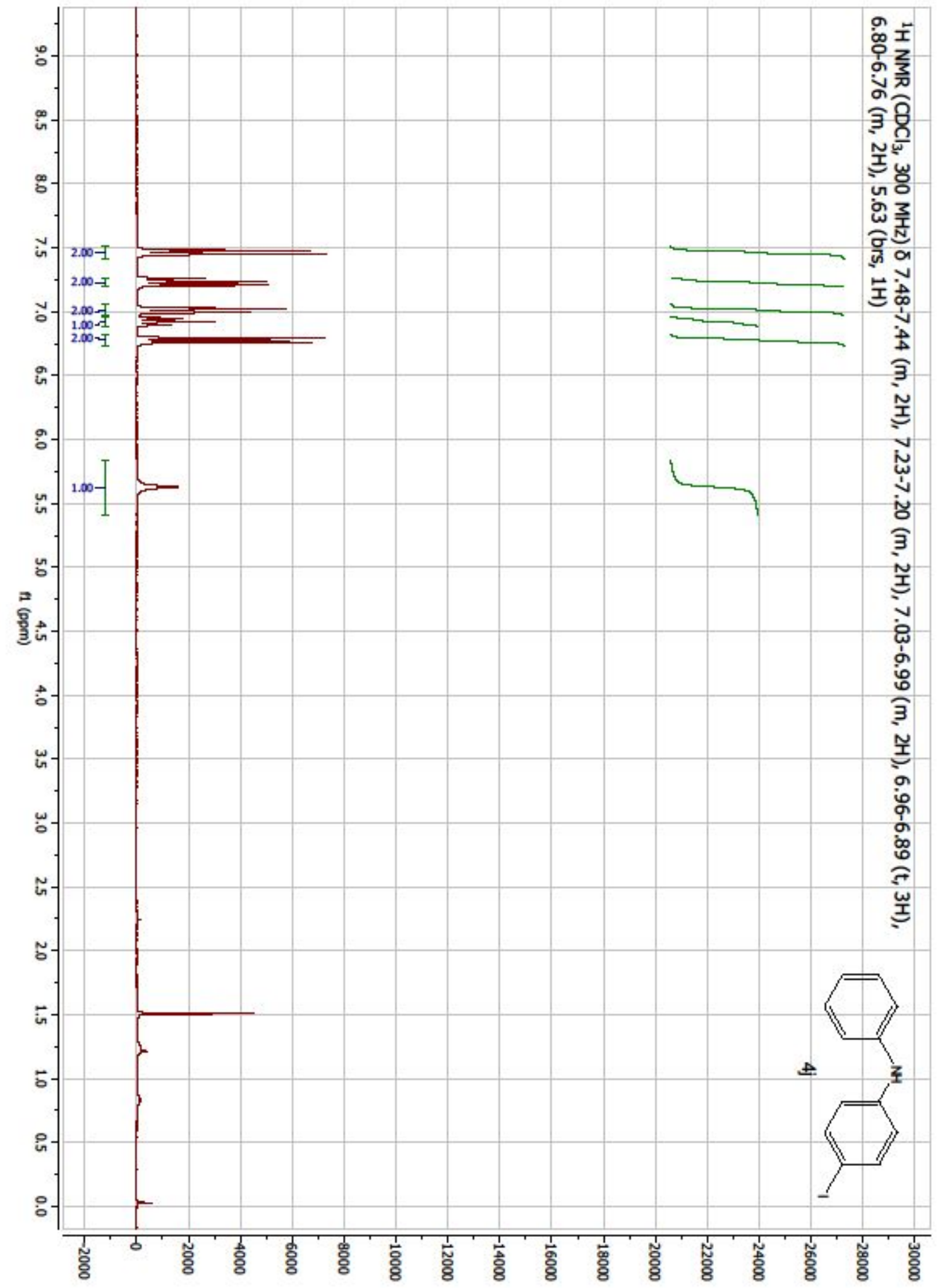




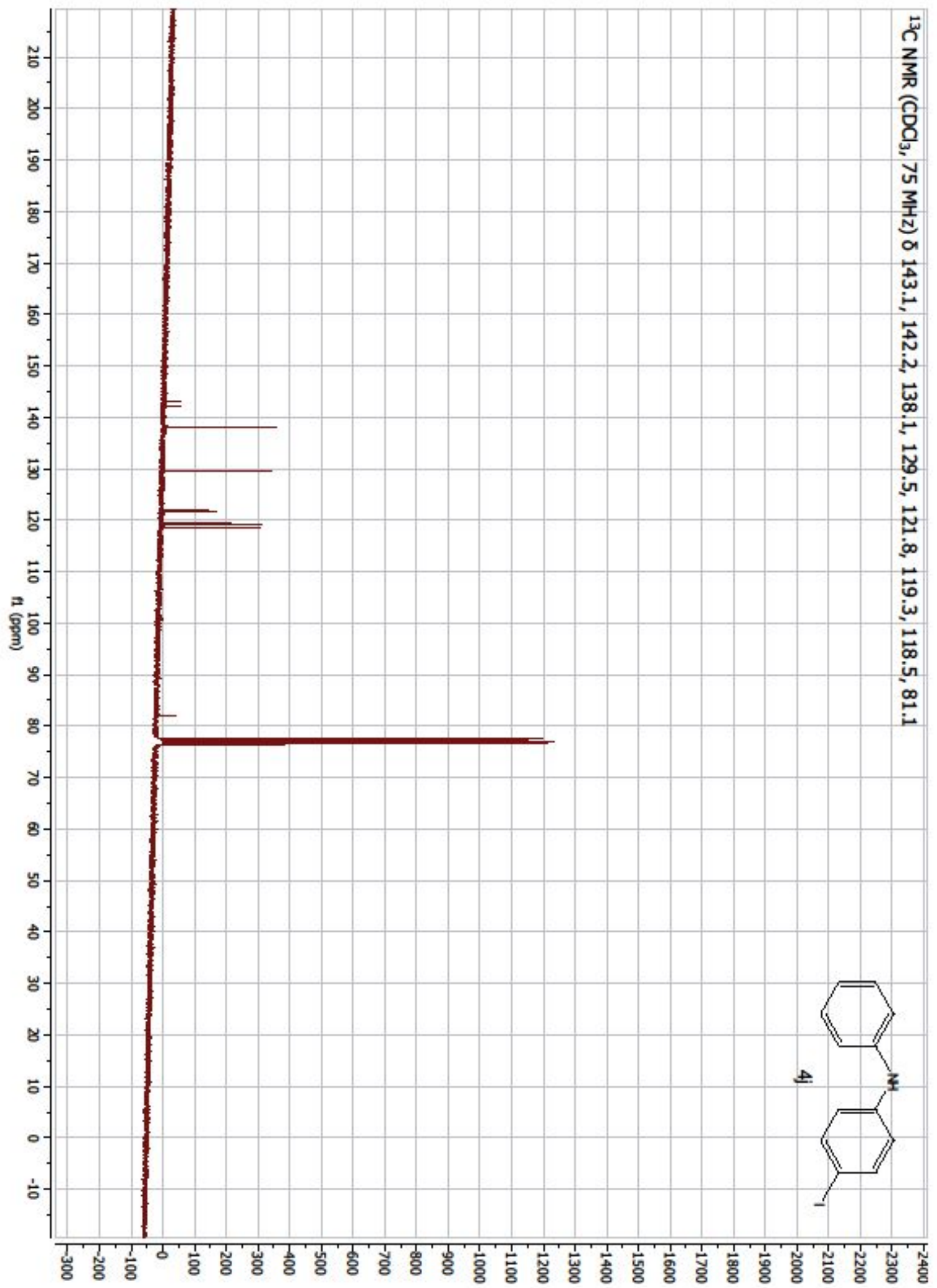




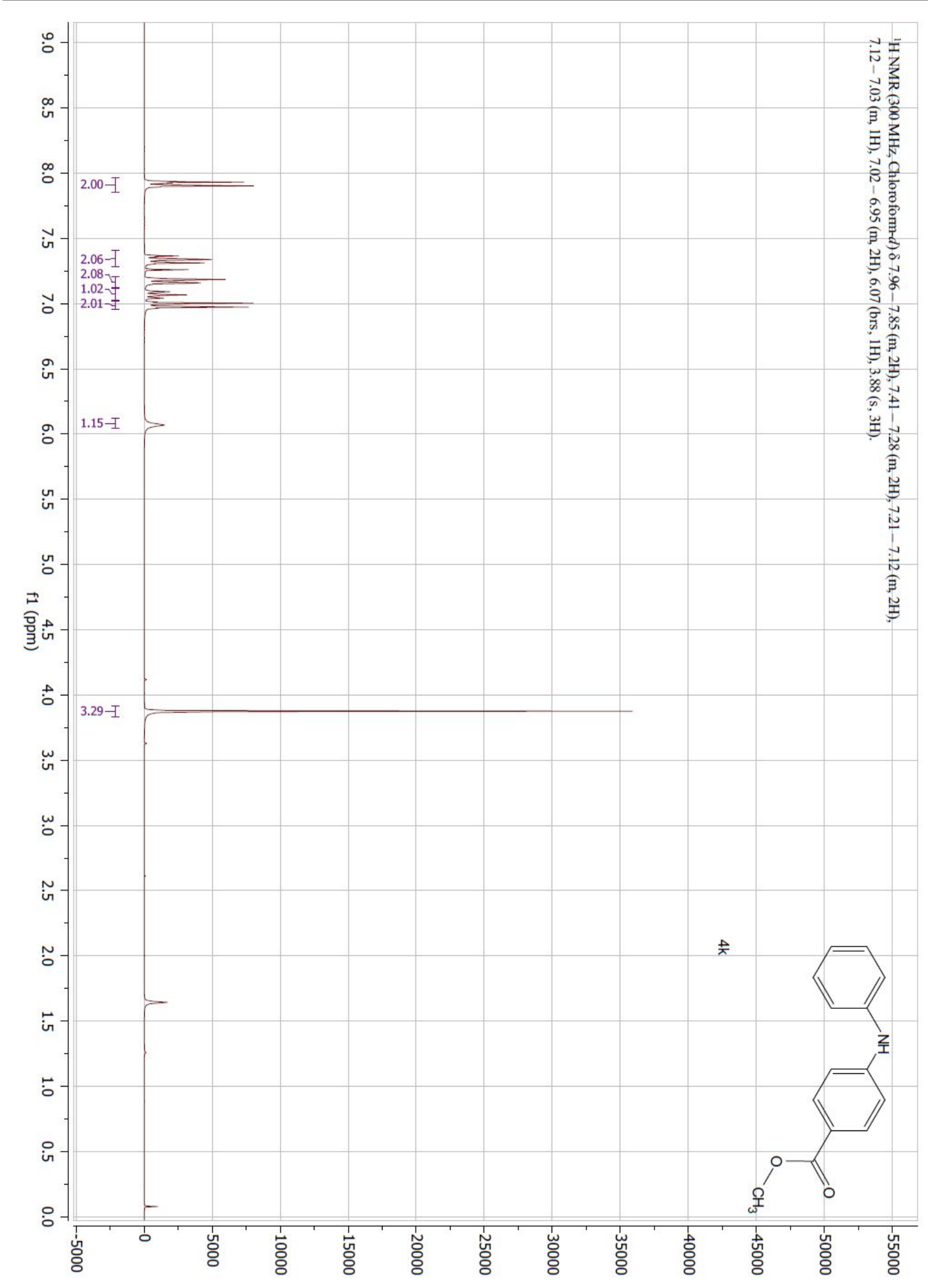




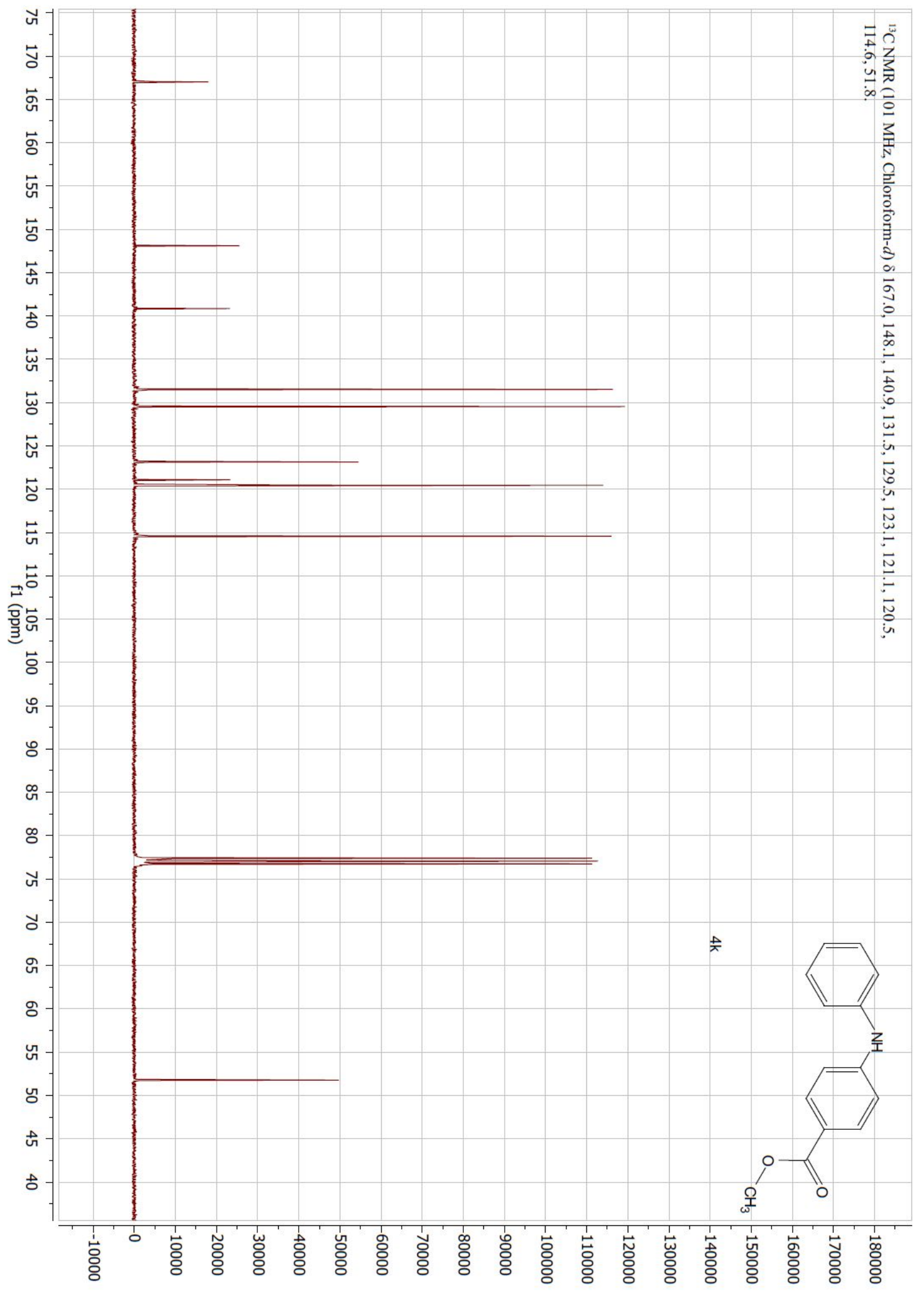




\section{References}

1. (a) Dale, W. J.; Rush, J. E. J. Org. Chem. 1962, 27, 2598; (b) Kabalka, G. W.; Sastry, U.; Sastry, K. A. R. J. Organomet. Chem. 1983, 259, 269.

2. Tamayo, A. B.; Alleyne, B. D.; Djurovich, P. I.; Lamansky, S.; Tsyba, I.; Ho, N. N.; Bau, R.; Thompson, M. E. J. Am. Chem. Soc. 2003, 125, 7377.

3. Nakamura, Y.; Ilies, L.; Nakamura, E. Org. Lett. 2011, 13, 5998.

4. Raghuvanshi, D. S.; Gupta, A. K.; Singh, K. N. Org. Lett. 2012, 14, 4326.

5. Altman, R. A.; Anderson, K. W.; Buchwald, S. L. J. Org. Chem. 2008, 73, 5167.

6. Maiti, D.; Fors, B. P.; Henderson, J. L.; Nakamura, Y.; Buchwald, S. L. Chem. Sci. 2011, 2, 57.

7. Tardiff, B. J.; Stradiotto, M. Eur. J. Org. Chem. 2012, 3972.

8. Kataoka, N.; Shelby, Q.; Stambuli, J. P.; Hartwig, J. F. J. Org. Chem. 2002, 67, 5553.

9. Lü, B.; Li, P.; Fu, C.; Xue, L.; Lin, Z.; Ma, S. Adv. Synth. Catal. 2011, 353, 100.

10. Sutter, M.; Duclos, M.-C.; Guicheret, B.; Raoul, Y.; Métay, E.; Lamaire, M. ACS Sustainable Chem. Eng. 2013, 1, 1463.

11. MacNeil, S. L.; Wilson, B. J.; Snieckus, V. Org. Lett. 2006, 8, 1133.

12. Yoo, W. J., Tsukamoto, T., \& Kobayashi, S. Angew.Chem. Int. Ed. 2015, 54, 6587-6590. 